M O N O G R A P I A E B O T A N I C A E

Vol. 70, 1988

GRAŻYNA H. TOMASZEWICZ

\title{
DESMIDS OF THE TRANSITIONAL BOGS OF THE MIDDLE MAZOWSZE LOWLAND
}

Desmidie torfowisk przejściowych Niziny Srodkowomazowieckiej

Redaktor: $R$. Olaczek

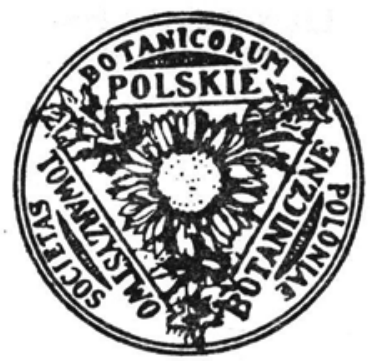




\section{Wydano z pomocą finansową}

Polskiej Akademii Nauk

\section{KOMITET REDAKCYJNY}

R. Olaczek (Lódź), E. Sidorkiewicz (Warszawa)

\section{RADA REDAKCYJNA}

W. Matuszkiewicz (Warszawa), Z. Podbielkowski (Warszawa), E. Sidorkiewicz (Warszawa), R. Olaczek (Łódź), H. Piotrowska (Gdańsk), K. Rostański (Katowice), W. Żukowski (Poznań)

Adres redakcji: Al. Ujazdowskie 4, 00-478 Warszawa

Copyright by Państwowe Wydawnictwo Naukowe, Warszawa 1988

ISBN 83-01-07336-5

ISSN $0077-0655$ 


\section{CONTENTS}

Introduction . . . . . . . . . . . . . . . . . . . . . . . . . 5

Location and description of bogs . . . . . . . . . . . . . . . . . . 5

Material and methods . . . . . . . . . . . . . . . . . . . . . 99 9

List of the identified taxa . . . . . . . . . . . . . . . . . . . . . . . 10

Streszczenie . . . . . . . . . . . . . . . . . . . . . . . 80

Literature . . . . . . . . . . . . . . . . . . . . . 81 


\section{ABSTRACT}

Grażyna H. Tomaszewicz. Desmids of the transitional bogs of the Middle Mazowsze Lowland. Monogr. Bot., vol. 70, 1987

Abstract. The author presents the results of the studies of several years on the desmid flora of nine transitional bogs located in the environs of Warsaw. 226 taxa were found belonging to 20 genera. Moreover, 16 taxa of the Mesotaeniaceae family, representing 4 genera were identified. A few taxonomic corrections were made in relation to several taxa. The rank within the species was changed or it was transferred to another genus. Certain species and distinguished infraspecific taxa were criticized. 


\section{INTRODUCTION}

Desmids are a group of algae which are interesting, very diversified in shape and with specific ecological requirements. Members of the most genera occur rather in stagnant, slightly acid waters with low calcium content. Therefore, a large number of taxa can be encountered in bogs, in small peaty pools or in lakes located in bogs. Eichler (1890) and Kozłowski (1895) were the first Polish authors who noticed that desmids were very abundant in these habitats.

So far, desmid flora has been described only for some bogs in Poland, and only when the whole algae flora has been studied. Most papers concern desmids of ombryotrophic bogs such as: Bielawskie Błoto (Krawiecowa et al. 1954), Nowy Targ Basin (Wasylik 1961a), Chlebowo (Gołowin 1964), and Bór na Czerwonem (Grzelewska 1974). Except for the investigations of Wysocka (1934) and Kadłubowska (1952) desmids of the transitional bogs have not been studied yet. Therefore, such studies are advisable and needed.

\section{LOCATION AND DESCRIPTION OF BOGS}

Nine transitional bogs (Biały Ług A, Biały Ług B, Celestynów, Konik, Bagno Jacka, Zieleniec, Grabicz A, Grabicz B and Mironowe Górki), located in the Middle Mazowsze Lowland, were studied. All of them, Celestynów bog located in the Garwolin Plain - except, were located in the Wołomin Plain mesoregion (Kondracki 1977).

Biały Ług A bog (stand 1) lies about $18 \mathrm{~km}$ southeast of Warsaw and about $2 \mathrm{~km}$ southwest of Wiązowna (Fig. 1). Its surface equals to app. 2 ha. It is surrounded by pine forest with the high contribution of lichens. The bog is intensively exploited. Peat excavations are mainly of different age. Old excavations are nearly completely regenerated. They are occupied by phytocenoses composed mainly of Sphagnum apiculatum and Eriophorum angustifolium as dominants and Oxycoccus quadripetalus and Eriophorum vaginatum. In new and deep excavations turfs of peat mosses draw from the margins inwards. In wide dykes and unexploited parts of the bog well-developed Eriophoro-Sphagnetum re- 
curvi phytocenoses with high contribution of Eriophorum vaginatum and Oxycoccus quadripetalus occur. Here and there, pine and birch, more or less abundant, grow. Water in this bog is highly acid, pH 3.4-4.5.

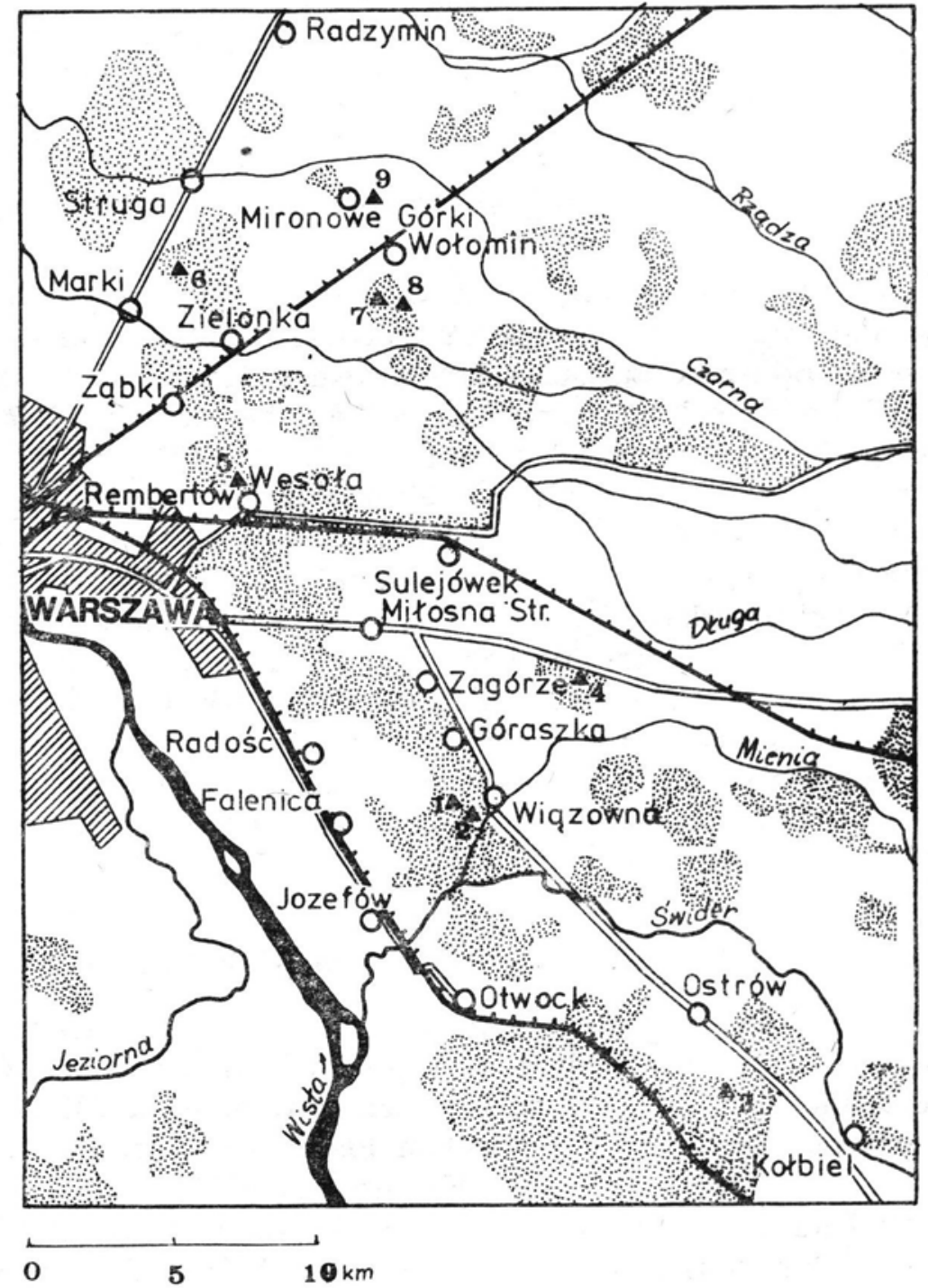

Fig. 1. Distribution of bogs studied. 1 - Biały Ług A, 2 - Biały Ług B, 3 - Celestynów, 4 - Konik, 5 - Bagno Jacka, 6 - Zieleniec, 7 - Grabicz A, 8 - Grabicz B, 9 - Mironowe Górki

Biały Ług B bog (stand 2) lies beyond the dune bank, about $1 \mathrm{~km}$ east of the Biały Ług A bog (Fig. 1). Its surface equals to app. 2.5 ha. It is surrounded by pine forest. The bog is highly exploited, as a rule to the floor, at present it is actually one, big, branched peat excavation. 
Most of the peat excavations are occupied by Caricetum elatae phytocenose, which in the bog margins is composed of Eriophorum angustifolium and Sphagnum apiculatum. Utricularia minor is very abundant in water. In the bridges between excavations and ditches, as well as in unexploited area small patches of Eriophoro-Sphagnetum recurvi phytocenoses occur. Single specimens of pine and birch also grow there. Water $\mathrm{pH}$ oscilates between 3.7 and 4.9 .

Celestynów bog (stand 3) is located about $33 \mathrm{~km}$ southeast of Warsaw and app. $1.5 \mathrm{~km}$ west of Anielinek (Fig. 1). Its surface equals to 8 ha. It is surrounded by pine forest. The bog was intensively exploited, at present peat excavations are regenerated to a large extent. Amongst water plants Nymphaea alba (in some excavations very abundant) and Utricularia minor grow there. The bottom is covered with Sphagnum cuspidatum. Water blooming of filamentous algae, mainly Mougeotia sp., often takes place. Floating bog-moss turf in the margins is composed of Sphagnum apiculatum. In the regenerated excavations $S p h$. apiculatum is accompanied by Rhynchospora alba, Eriophorum angustifolium and Drosera rotundifolia, sometimes in large quantities. In the bridges and unexploited parts patches of bog pine forest with higher contribution of Ledum palustre, Vaccinium uliginosum, Oxycoccus quadripetalus and Eriophorum vaginatum occur. Water $\mathrm{pH}$ equals 3.4-4.8.

Konik bog (stand 4) lies $23 \mathrm{~km}$ southeast of Warsaw (Fig. 1). Its surface equals to app. 8 ha. It is surrounded by the young pine forest. It was intensively exploited in past. Most of the peat excavations are covered with floating bog-moss turf built mainly of Sphagnum apiculatum and Eriophorum angustifolium. Free water-level is maintained only in the youngest and deepest bodies. Amongst flowering plants Utricularia minor grows abundantly there. Periodically, turfs of filamentous algae, mainly Spirogyra sp. and Mougeotia sp., develop there in mass. In the excavations in the bog margin, where considerable inflow of eutrophic waters takes place, Phragmitetum and Typhetum latifoliae phytocenoses occur. In some old, regenerated excavations Caricetum lasiocarpae phytocenoses often grow. In the unexploited bog parts patches of Eriophoro-sphagnetum recurvi are found. The bog is scarcely (mainly in the bridges between excavations) grown with Betula pubescens. In the margines larger clumps of Salix cinerea and Frangula alnus may be found. Water $\mathrm{pH}$ ranges from 3.5 to 5.0 .

Bagno Jacka bog (stand 5) is located $14 \mathrm{~km}$ east of Warsaw (Fig. 1) and $1 \mathrm{~km}$ northwest of Wesoła. Its surface equals to over $35 \mathrm{ha}$. It is surrounded by the forest complex (young pine forests). The bog was intensively exploited in past. Old excavations are nearly entirely shallowed and overgrown. In the young and shallow parts slightly compact phytocenoses of Caricetum elatae occur, in the deeper parts water-surface is free. In the margins of excavations, in shallowed parts, small-surface 
patches of Menyanthes trifoliata and Calla palustris grow, as well as small bog-moss bridges, composed mainly of Sphagnum apiculatum, occur. In old, nearly entirely regenerated peat excavations moss layer is composed mainly of Sphagnum apiculatum, whereas amongst flowering plants Carex rostrata, Eriophorum angustifolium and Oxycoccus quadripetalus mostly grow. The unexploited part of the bog is occupied by classicly developed Eriophoro-Sphagnetum recurvi phytocenoses with high contribution of young pine. In some spots they pass into bog pine forest. In some bog parts Betula pubescens occurs abundantly. Water $\mathrm{pH}$ amounts to 4.0-5.0.

Zieleniec bog (stand 6) is located $13 \mathrm{~km}$ northeast of Warsaw (Fig. 1) and $2 \mathrm{~km}$ north of Zielonka. Its surface exceeds $2.5 \mathrm{ha}$. It is surrounded by young pine forest. The bog has been highly exploited. Old excavations are overgrown with Sphagnum apiculatum accompanied by Eriophorum angustifolium and E. vaginatum in large quantities. In young excavations with free water-surface the bottom is covered with Sphagnum cuspidatum, whereas in the margins Heleocharis palustris, Eriophorum angustifolium, Carex lasiocarpa and $C$. hudsonii sometimes grow. In the bridges between excavations degenerated phytocenoses of bog pine forest with Molinia coerulea and Calluna vulgaris occur. Vaccinium uliginosum, V. vitis-idaea, Carex canescens, Calamagrostis canescens, Betula pubescens, Populus tremula and Frangula alnus grow in smaller quantities. Water $\mathrm{pH}$ oscilates between 3.7 and 4.8 .

Grabicz A bog (stand 7) lies $17 \mathrm{~km}$ nôrtheast of Warsaw and $1.5 \mathrm{~km}$ south of Wołomin (Fig. 1). Its surface equals to about 5 ha. It is surrounded by young pine forest. The bog hardly exploited, with the low peat depth. In water of the deepest, probably natural depression agglomerations of Nymphaea alba, Potamogeton natans, Sphagnum subsecundum var. auriculatum and Phragmitetum phytocenoses occur. The bog is mostly covered with Caricetum lasiocarpae phytocenoses with large amounts of Sphagnum apiculatum. The following species are mostly found within the bog: Comarum palustre, Eriophorum angustifolium, Lysimachia vulgaris, Salix rosmarinifolia, Vaccinium uliginosum and Pinus sylvestris. The bog vegetation is of the moss-sedge nature and refers to the Scheuchzerio-Caricetea fuscae class with the distinct developmental tendencies towards bog-moss communities of the Oxycocco-Sphagnetea class. Water is slightly acid, $\mathrm{pH}=4.2-6.0$.

Grabicz B bog (stand 8) lies beyond the dune bank, $1 \mathrm{~km}$ east of Grabicz A bog (Fig. 1). Its surface equals to $13 \mathrm{ha}$. It is nearly entirely surrounded by young pine forest. It is intensively exploited and in many parts the peat was skimmed to the floor. In the deeper parts of free peat excavations Phragmitetum phytocenoses occur, whereas in the shallow parts patches of Caricetum elatae grow. In water single specimens of $\mathrm{Nym}$ phaea alba may be found, while Drepanocladus fluitans occurs abun- 
dantly. In the shallowed margin parts bog-moss bridges develop, composed mainly of Sphagnum apiculatum and with such species as: Lysimachia vulgaris, L. thyrsiflora, Carex rostrata, Comarum palustre, Eriophorum angustifolium and Calamagrostis canescens. In the bog bridges between excavations degenerated phytocenoses of the bog pine forest grow with high contribution of Vaccinium uliginosum, Salix cinerea and Molinia coerulea. The regeneration of the peat excavations goes in the direction of moss-sedge and bog-moss-sedge communities. Water $\mathrm{pH}$ equals to $3.8-5.0$.

Mironowe Górki bog (stand 9) located $19 \mathrm{~km}$ northeast of Warsaw, in the Wołomin suburb (Fig. 1). Its surface equals to over 25 ha. The bog is surrounded by settlements and arable fields. It is highly exploited and large-surface peat excavations are of different age. Water vegetation is poorly developed, only single specimens of Nymphaea alba and Potamogeton natans occur. In some excavations Utricularia minor is more abundant. Fen vegetation is mostly represented by variously developed (more or less compact) Caricetum elatae phytocenoses. In some places the patches of Phragmitetum and Caricetum rostratae occur. In old shallowed peat excavations, with no free water-surface mainly communities composed of Sphagnum apiculatum - a dominant, and Eriophorum angustifolium, Carex rostrata, C. hudsonii grow. In the bridges and bog margins sometimes Salix cinerea, Betula pubescens, B. verrucosa occur in number accompanied by numerous species of fen plants and those confined to moist habitats. Water is slightly acid, $\mathrm{pH}=4.2-5.6$.

\section{MATERIAL AND METHODS}

The material was collected in the period from October 1971 to December 1976 in more or less month intervals, with the exception of winter months.

The samples in particular bogs were collected in various habitats and different ways, mostly:

a) the water was squeezed from submerged bog mosses, mats of filamentous algae (Mougeotia sp., Spirogyra sp.) and Utricularia minor agglomerations;

b) surface layer sediment was collected from the bottom of shallow peat excavations, drains and other depressions;

c) periphyton was scraped out of the partly submerged plants or of those with floating leaves (Phragmites communis, Nymphaea alba);

d) over $20 \mathrm{dm}^{3}$ of water from peat excavations, drains and other depressions, constant or periodically dried were filtered through plankton-net No 25.

In aim to find large number of taxa in each bog samples were collected from at least 10 various points. The frequency of occurrence of 
each taxon was determined as from each bog there were some scores of samples. The following system and abbreviations were assumed: cc very often, $\mathrm{c}$ - often, $\mathbf{r}$ - relatively often, rr - rarely, rrr - sporadically.

In aim to cover variation in the size of individuals, minimum 10 specimens were measured for each taxon, moreover, a special attention was paid to the largest and the smallest specimens. In case of rare species all encountered specimens were measured.

\section{LIST OF THE IDENTIFIED TAXA}

In the taxa identification mainly following works were used: Ralfs (1848), West, West (1904, 1905, 1908, 1912), Migula (1907), West et al. (1923), Krieger (1933a, b, 1935, 1937a, b, 1939), Kossinskaya (1952, 1960), Teiling (1954, 1967), Hirano $(1955,1956,1957 \mathrm{a}, \mathrm{b}, 1959 \mathrm{a}$, b, 1960), Krieger, Gerloff (1962, 1965, 1969), Bicudo (1969), Förster (1970, 1972), Rủžička (1972, 1973a, 1977, 1981), Palamar-Mordvintseva (1982). The following works were used as well: Raciborski (1895), Skuja (1928), Kanetsuna (1960), Thomasson (1960), Růžička (1962, 1975b, 1976), Grönblad (1963), Ito (1965c, 1978), Scott et al. (1965), Dubois-Tylski (1966), Messikommer (1968/72), Lenzenweger (1970b, 1976), Coesel (1975), Palamar-Mordvintseva (1975b, 1977), Agarkar et al. (1979), Hirano (1979).

\section{Abbreviations:}

$\begin{array}{llll}\text { lg. } & \text { - cell length } & \text { pr. } & \text { - processes } \\ \text { br. } & \text { - cell breadth } & \text { ang. } & \text { - angular } \\ \text { br. max. } & \text { - maximum cell breadth } & \text { str. } & \text { - striae } \\ \text { isth. } & \text { - isthmus breadth } & \text { th. } & \text { - thickness of cell } \\ \text { lob. pol. } & \text { - polar lobe } & \text { occur. } & \text { - occurrence } \\ \text { ap. } & \text { - apex } & 1-9 & \text { - a stand (bog) number } \\ \text { sp. } & \text { - spine } & & \end{array}$

List of taxa contains additionally representatives of the family Mesotaeniaceae.

\section{Order: ZYGNEMAT ALES}

Family: Mesotaeniaceae Oltmanns 1904

Genus: Mesotaenium Nägeli 1849

Mesotaenium de-greyi Turn.

var. de-greyi f. de-greyi (Plate 1, Fig. 10-11)

Cells naked, without gelatinous envelope. They occur mainly in samples obtained through Sphagnum sp. squeezing. 
Lg. $78.2-96.6 \mu \mathrm{m}$, br. $18.4-20,7 \mu \mathrm{m}$, lg.: br. $3.89-4.67 \times$.

Occur.: 1rrr, 2rrr, 3rr, 4rrr, 5rrr, 6rrr, 8rrr, 9rrr.

f. breve (W. West) Kossinsk. (Plate 1, Fig. 8-9)

Entire cells filled with oil globules often (Plate 1, Fig. 9). Similar specimens have been described by West, West (1904) and Kossinskaya (1952). Individuals found both in plankton and in the Sphagnum sp. turf.

Lg. 36.8-59.8 $\mu \mathrm{m}$, br. 16.1-23.0 $\mu \mathrm{m}$, lg.: br. 1.70-3.00 ×.

Occur.: 1rrr, 2rrr, 3rr, 4rr, 5rr, 6rrr, 8rr, 9rrr.

Mesotaenium endlicherianum Näg.

var. endlicherianum f. endlicherianum (Plate 1, Fig. 3-5)

One specimen was found in the Bialy Lug $\mathbf{A}$ bog at the divisional stage (Plate 1, Fig. 5).

Lg. $32.2-46.0 \mu \mathrm{m}$, br. 9.2-11.5 $\mu \mathrm{m}$, lg.: br. 2.85-4.02 $\times$.

Occur.: 1rrr, 3rr, 8rrr, 9rr.

f. exiguum (Hansg.) stat. nov. Plate 1, Fig. 6-7)

Basion.: Mesotaenium endlicherianum Näg. var. exiguum Hansgirg (1892), Prodromus der Algenflora von Böhmen, Archiv Naturwiss. Landesdurchf. Böhmen, 8, 4, p. 248.

Specimens slightly curved, larger than those described by Hansgirg (1892), and Migula (1907). Krieger (1933b) considers this taxon as a synonym of the typical variety. I take it for an individual taxonomic unit. Cells differ considerably in shape from the typical form. No intermediate forms have been found. Representatives of both taxa (f. endlicherianum and f. exiguum) were recorded in separate sites (stands).

I propose to give the taxon mentioned above the rank of a form, not a variety.

Lg. $45.7-47.1 \mu \mathrm{m}$, br. $11.5 \mu \mathrm{m}$, lg.: br. $3.97-4.09 \times$.

Occur.: 2rrr.

f. grande (Nordst.) Kossinsk. (Plate 1, Fig. 1-2)

Lg. 46.0-94.3 $\mu \mathrm{m}$, br. 9.2-16.1 $\mu \mathrm{m}$, lg.: br. $4.71-5.81 \times$.

Occur.: 1rrr, 3rr, 5rrr, 6rrr, 8rrr, 9rrr.

Mesotaenium macroccocum (Kütz.) Roy et Biss.

var. macrococcum (Plate 1, Fig. 12-13)

Solitary cells without gelatinous envelope. Specimens were found in the plankton of the excavation only.

Lg. 28.6-34.5 $\mu \mathrm{m}$, br. 11.5-13.8 $\mu \mathrm{m}$, lg.: br. $2.48-2.50 \times$.

Occur.: 2rr.

Genus: Spirotaenia Brébisson in Ralfs 1848

Spirotaenia condensata Bréb.

var. condensata (Plate 1, Fig. 14)

Lg. 184.0-246.0 $\mu \mathrm{m}$, br. $20.7-24.6 \mu \mathrm{m}$, lg.: br. $8.38-10.00 \times$.

Occur.: 7rrr, 8rrr. 
Spirotaenia obscura Ralfs

var. obscura, morpha (Plate 1, Fig. 15)

Cells fusiform. Chromatophore not reaching the cell poles. Chromatophore ridges spirally twisted in two directions: from the left to the right and from the right to the left, in end they fuse with each other.

The comparison of drawings of Sp. obscura in the works of: Lütkemüller (1895; after West, West 1904 and Kossinskaya 1952), Migula (1907), Krieger (1933b), Grönblad (1938), Hirano (1955), Růžička (1957b), Grönblad and Růžička (1959), Wasylik (1961a) and Bicudo (1969) shows high variation of individuals of this taxon, both in respect of cell shape and chromatophore structure. Drawings in many works e.g. those of Migula (1907; Plate 24, Fig. 2), Krieger (1933b), Grönblad (1938), Růžička (1957b) and Bicudo (1969) differ from those of Lütkemüller (1895) who has thoroughly analysed chromatophore structure on the grounds of the culture material.

In the specimens found chromatophore is slightly different from that described by Lütkemüller and similar to those described by: Migula (1907), Růžička (1957b), Grönblad and Růžička (1959).

Lg. 75.9-80.5 $\mu \mathrm{m}$, br. 16.1-17.2 $\mu \mathrm{m}$, lg.: br. 4.68-4.71 $\times$.

Occur.: 7rrr.

Genus: Cylindrocystis (Meneghini ex Ralfs) De Bary 1858

Cylindrocystis brebissonii (Mengh. ex Ralfs) De Bary (Plate 2, Fig. 4-5)

Two varieties, brebissonii and jenneri have been distinguished within a present species. Both taxa can be identified on the grounds of the formation and appearance of zygospore. In the obtained material no individuals reproducing sexually were found. Therefore, their closer taxonomical affiliation could not be determined.

Lg. 39.1-75.9 $\mu \mathrm{m}$, br. 13.8-23.0 $\mu \mathrm{m}$, lg.: br. 2.00-4.16 $\times$.

Occur.: 1rrr, 2rr, 3rr, 4r, 5rrr, 6rrr, 8rrr.

Cylindrocystis crassa De Bary

var. crassa (Plate 2, Fig. 6)

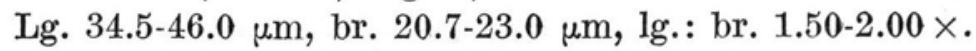

Occur.: 1rrr, 2rr, 3rrr, 4rr, 5rrr, 8rrr, 9rrr.

Genus: Netrium (Nägeli) Itzigsohn et Rothe 1856

Netrium digitus (Ehrenb. ex Bréb.) Itzigs. et Rothe

var. digitus f. digitus (Plate 1, Fig. 16)

Lg. 110.4-267.3 $\mu \mathrm{m}$, br. 31.0-73.6 $\mu \mathrm{m}$, ap. 16.1-32.2 $\mu \mathrm{m}$, lg.: br. $3.10-4.76 \times$.

Occur.: 1rr, 2rr, 3rr, 4r, 5rr, 6rr, 7rrr, 8rr, 9rr.

? f. curtum (Anderss.) Kossinsk. (Plate 1, Fig. 17) 
Individuals differ in size from those described by Krieger (1933b) and Kossinskaya (1952). Cell dimensions are close to those of f. digitus, whereas length-breadth ratio is comparable to that of $f$. curtum.

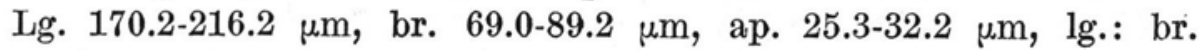
$2.33-2.56 \times$.

Occur.: 4rrr, 5rrr.

\section{f. parvum Borge (Plate 1, Fig. 18)}

Cells fusiform. They fit their drawings of Parra and Gonzalez (1977; Fig. 8), slightly differ from others (Fig. 6-7 and 9), as well as from Borge's drawing reproduced in works of Krieger (1933b) and Kossinskaya (1952). The drawings mentioned there in their shape resemble a rhombus. It may well be that specimens found represent only juvenile stages of f. digitus and not separate taxon.

Lg. 69.0-87.4 $\mu \mathrm{m}$, br. 27.6-32.2 $\mu \mathrm{m}$, ap. 11.5-13.8 $\mu \mathrm{m}$, lg.: br. 2.45-3.05×.

Occur.: 5rrr, 8rrr, 9rrr.

var. lamellosum (Bréb) Grönbl. (Plate 1, Fig. 19-21)

Specimens found are morphologically diversified and can be divided into three groups:

a) Cells similar in shape and dimensions to the majority of described specimens of var. lamellosum e.g. Ito (1966a), Grönblad et al. (1968), Bicudo (1969), Förster (1970), Růžička (1973a), (Plate 1, Fig. 19)

Lg. 202.4-307.1 $\mu \mathrm{m}$, br. 41.4-57.5 $\mu \mathrm{m}$, ap. 18.4-29.9 $\mu \mathrm{m}$, lg.: br. $4.55-6.52 \times$.

Occur.: 2rrr.

b) morpha 1 (Plate 1, Fig. 20)

Cells small considerably differ in dimensions from those mentioned above. Small specimens of var. lamellosum have been described, among others, by Raciborski (1892), Krieger (1933a), Woronichin (1950 after Kossinskaya 1952), Croasdale (1955 after Förster 1972), Förster (1972) and Agarker, Agarkar (1977). Krieger (1933b) and Ko ssinskaya (1952) have included them in the present variety and do not form a special taxon of the lower rank. At the same time, they considerably widen the range of cell dimensions typical of var. lamellosum individuals. Förster (1972) has distinguished small specimens into formae minores. He has stated they differ not only in size, but also in more narrow apices and more shallow median constriction.

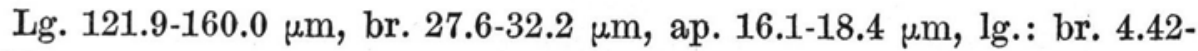
$-5.20 \times$.

Occur.: 2rrr.

c) morpha 2 (Plate 1, Fig. 21)

Cells longitudinally fusiform, margines in the centre straight parallel, without median constriction. Specimens fit description and drawings of var. lamellosum in the papers of: Hirano (1955), Ito (1965b), Kanetsuna (1967) and Goto (1975). 
PLATE 1

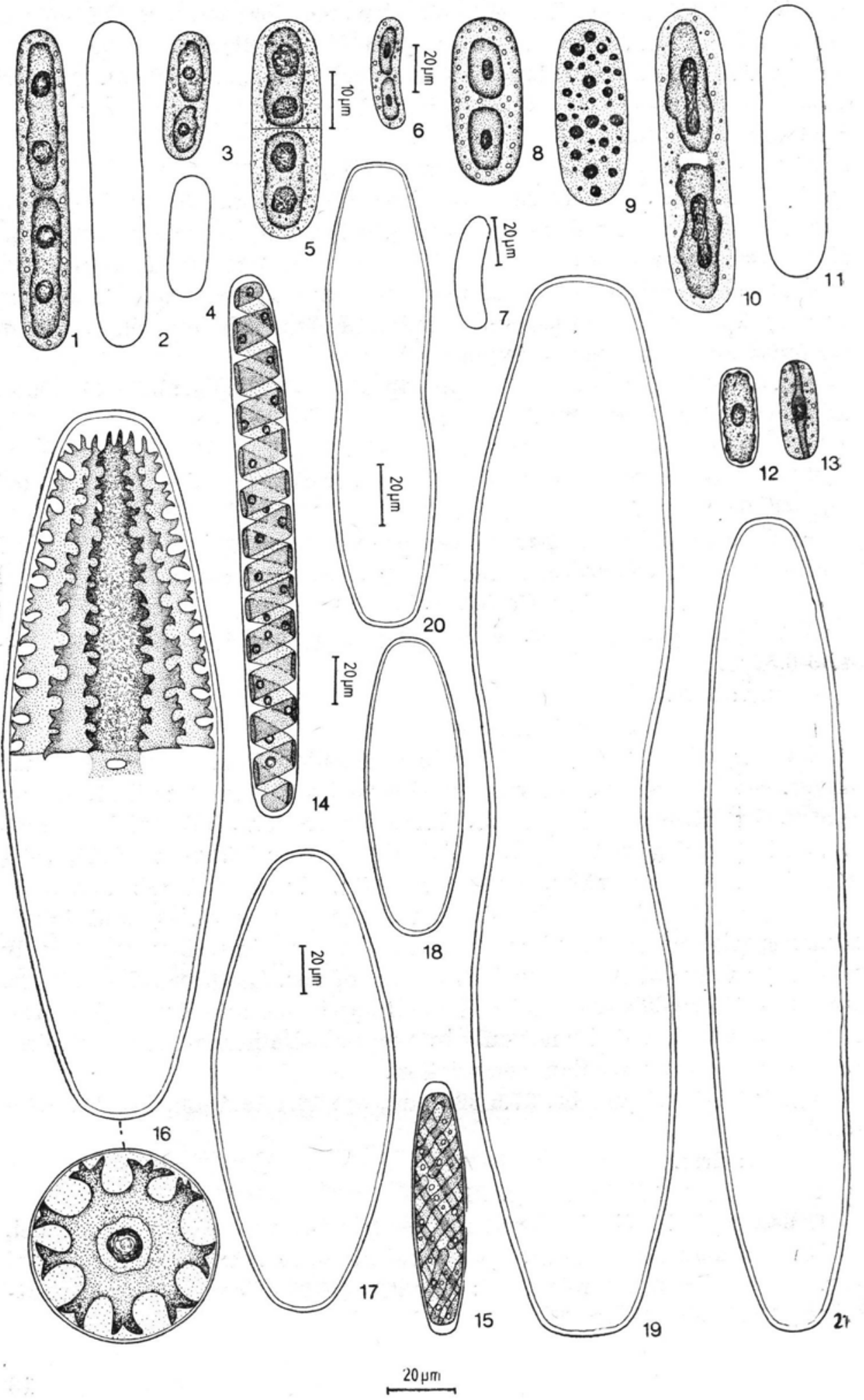




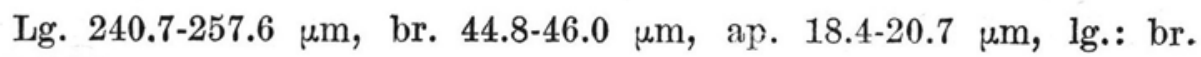
$5.30-5.60 \times$.

\section{Occur.: 2rrr.}

I classify all three groups into one taxon, considering differences between them to be caused by intrataxon variation. Representatives of all three groups were found in all samples.

Netrium oblongum (De Bary) Lütkem.

var. oblongum (Plate 2, Fig. 1)

Lg. 117.3-121.9 $\mu \mathrm{m}$, br. $31.0-36.8 \mu \mathrm{m}$, lg.: br. $3.22-3.79 \times$.

Occur.: 3rrr.

var. cylindricum W. et G. S. West (Plate 2, Fig. 2-3) Fig. 2)

a) Specimens fit those described in the relevant literature (Plate 2,

Lg. 87.4-109.2 $\mu \mathrm{m}$, br. 26.4-27.6 $\mu \mathrm{m}$, lg.: br. $3.17-4.13 \times$

Occur.: 3rrr.

b) morpha (Plate 2, Fig. 3)

Cells bigger and relatively longer than typical of var. cylindricum. Dimensions considerably differ from those described by: West, West (1904), Roll (1923), Taylor (1934), and Hirano (1955). Cell length similar to maximum dimensions given by Krieger (1933b) and Růžička (1954).

Lg. 144.9-188.6 $\mu \mathrm{m}$, br. 29.9-32.2 $\mu \mathrm{m}$, lg.: br. $4.85-5.86 \times$.

Occur.: 3rrir.

\section{Order: DESMIDIALES}

Suborder: CLOSTERIINEAE

Family: Gonatozygaceae (Lütkemüller) G. S. West et F.E. Fritsch 1927

\section{Genus: Gonatozygon De Bary 1856}

Gonatozygon brebissonii De Bary

var. brebissonii (Plate 2, Fig. 7)

\section{Plate 1}

Fig. 1-2. Mesotaenium endlicherianum var. endlicherianum f. grande. Fig. 3-5. Mes. endlicherianum var. endlicherianum f. endlicherianum. Fig. 6-7. Mes. endlicherianum var. endlicherianum f. exiguum. Fig. 8-9. Mes. de-greyi var. de-greyi f. breve. Fig. 10-11. Mes. de-greyi var. de-greyi f. de-greyi. Fig, 12-13. Mes. macrococcum var. macrococcum. Fig. 14. Spirotaenia condensata var. condensata. Fig. 15. Sp. obscura var. obscura, morpha. Fig. 16. Netrium digitus var. digitus f. digitus. Fig. 17. ?N. digitus var. digitus f. curtum. Fig. 18. N. digitus var. digitus f. parvum. Fig. 19. N. digitus var. lamellosum. Fig. 20. N. digitus var. lamellosum, morpha 1. Fig. 21. N. digitus var. lamellosum, morpha 2 


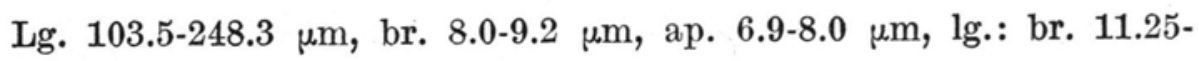
$-26.99 \times$.

Occur.: 7rrr, 8rrr.

var. kjellmanii (Wille) Racib. (Plate 2, Fig. 8)

Lg. 57.5-58.6 $\mu \mathrm{m}$, br. $8.0 \mu \mathrm{m}$, ap. $5.7 \mu \mathrm{m}$, lg.: br. $7.19-7.33 \times$.

Occur.: 7rrr.

\section{Family: Peniaceae Haeckel 1894}

Genus: Penium Brébisson in Ralfs 1848

Penium exiguum W. West

var. exiguum f. exiguum (Plate 2, Fig. 15)

Lg. 20.7-28.7 $\mu \mathrm{m}$, br. 6.9-8.0 $\mu \mathrm{m}$, ap. 5.7-6.9 $\mu \mathrm{m}$, lg.: br. 3.00-4.16 $\times$.

Occur.: 2rrr, 8rrr, 9rri.

f. major W. et G. S. West (Plate 2, Fig. 16)

Krieger (1935) and Růžička (1977) include this taxon into synonym of the typical taxon, whereas Kossinskaya (1960) treats it as a separate one.

In the material obtained no intermediate forms were found between specimens of f. exiguum and f. major. Individuals classified as f. major differ in dimensions from those classified as f. exiguum. Therefore, I accept Kossinskaya's (1960) view point and I distinguish f. major.

Lg. 57.5-67.8 $\mu \mathrm{m}$, br. 11.5-13.8 $\mu \mathrm{m}$, ap. 9.2-11.5 $\mu \mathrm{m}$, lg.: br. 4.91-5.74 $\times$.

Occur.: 2rrr.

var. capitatum (Roll) Kossinsk. (Plate 2, Fig. 17)

Lg. 39.1-43.7 $\mu \mathrm{m}$, br. 11.5-13.8 $\mu \mathrm{m}$, ap. 9.2-11.5 $\mu \mathrm{m}$, lg.: br. 3.08-3.47 $\times$.

Occur.: 2rrr.

Penium polymorphum (Perty) Perty

var. polymorphum (Plate 2, Fig. 11)

Lg. $42.5-43.7 \mu \mathrm{m}$, br. 24.2-25.3 $\mu \mathrm{m}$, isth. 23.0-24.2 $\mu \mathrm{m}$, lg.: br. 1.73$-1.76 \times$, str. $15-16 / 10 \mu \mathrm{m}$.

Occur.: 3rrr.

Penium silvae-nigrae Raban.

var. silvae-nigrae (Plate 2, Fig. 9)

Lg. 48.3-57.5 $\mu \mathrm{m}$, br. 24.1-25.3 $\mu \mathrm{m}$, isth. 21.8-24.1 $\mu \mathrm{m}$, lg.: br. $2.00-$ $-2.39 \times$, str. $7 / 10 \mu \mathrm{m}$.

Occur.: 1rrr, 3rrr.

var. parallehum W. Krieg. (Plate 2, Fig. 10)

Lg. $64.4 \mu \mathrm{m}$, br. $18.4 \mu \mathrm{m}$, isth. $17.2 \mu \mathrm{m}$, lg.: br. $3.50 \times$, str. $10 / 10 \mu \mathrm{m}$.

Occur.: 1rrr.

Penium spirostriolatum Bark.

var. spirostriolatum (Plate 2, Fig. 12-13) 
Lg. 133.4-220.8 $\mu \mathrm{m}$, br. 18.4-23.0 $\mu \mathrm{m}$, isth. 16.1-20.7 $\mu \mathrm{m}$, ap. 11.5$17.2 \mu \mathrm{m}, \mathrm{lg} .:$ br. $6.56-10.67 \times$.

Occur.: 7r, 8rrr, 9rrr.

var. amplificatum Schmidt (Plate 2, Fig. 14)

Specimens more slender than those described by Kossinskaya (1960).

Lg. 184.0-302.9 $\mu \mathrm{m}$, br. 17.2-21.8 $\mu \mathrm{m}$, isth. 17.2-18.4 $\mu \mathrm{m}$, ap. 11.5-16.1 $\mu \mathrm{m}$, lg.: br. $8.89-15.76 \times$.

Occur.: 4rrr.

\section{Family: Closteriaceae Pritchard 1852}

\section{Genus: Closterium Nitzsch ex Ralfs 1848}

Closterium abruptum W. West

var. abruptum f. angustissimum Schmidle (Plate 4, Fig. 10)

Lg. 117.3-167.9 $\mu \mathrm{m}$, br. 8.0-9.2 $\mu \mathrm{m}$, ap. 3.4-4.6 $\mu \mathrm{m}$, lg.: br. 14.66-19.55 $\times$.

Occur.: 7rrr, 8rrr, 9rrr.

Closterium acutum Bréb.

var. acutum (Plate 2, Fig. 23-24)

The most specimens in the samples were small (Plate 2, Fig. 24), so difficult to identify. I have never been sure if they belong to the typical variety, or if they are those of var. tenuius (Nordst.) W. Krieg. Lower limit of dimensions of var. acutum specimens is quoted differently by various authors e.g. West, West (1904), Krieger (1933a, 1935), Hirano (1955), Kossinskaya (1960), Rủžička (1972), Sarim, Faridi (1976) and Lind, Brook (1980).

There were no distinct habitat differences between the extreme (the smallest and the biggest) individuals. To the contrary, they occured in the same samples in Grabicz A stand.

Just as Rưžička (1977), I consider that to maintain taxon var. tenuius as a distinct taxonomical unit is not justified. It should be treated as a synonym of var. acutum, at the same time variation range of the typical taxon ought to be widened.

Lg. 65.5-105.8 $\mu \mathrm{m}$, br. 2.3-4.6 $\mu \mathrm{m}$, ap. $\sim 1 \mu \mathrm{m}$, lg.: br. 20.00-35.00 $\times$.

Occur.: 2rrr, 6rrr, 7c, 9rr.

var. linea (Perty) W. et G. S. West (Plate 2, Fig. 25-26)

In the bog Grabicz A, besides typical specimens some narrower specimens were observed. Therefore, within the taxon two groups of individuals were distinguished:

a) Specimens which fit the literature data (Plate 2, Fig. 25)

Lg. 82.8-151.8 $\mu \mathrm{m}$, br. 3.4-4.6 $\mu \mathrm{m}$, ap. $\sim 1 \mu \mathrm{m}$, lg.: br. 21.50-35.18 $\times$. Occur.: 1rrr, 2rrr, 3rrr, 4rr, 5rrr, 6rr, 7c, 8rr, 9c,

b) morpha (Plate 2, Fig. 26) 
PLATE 2
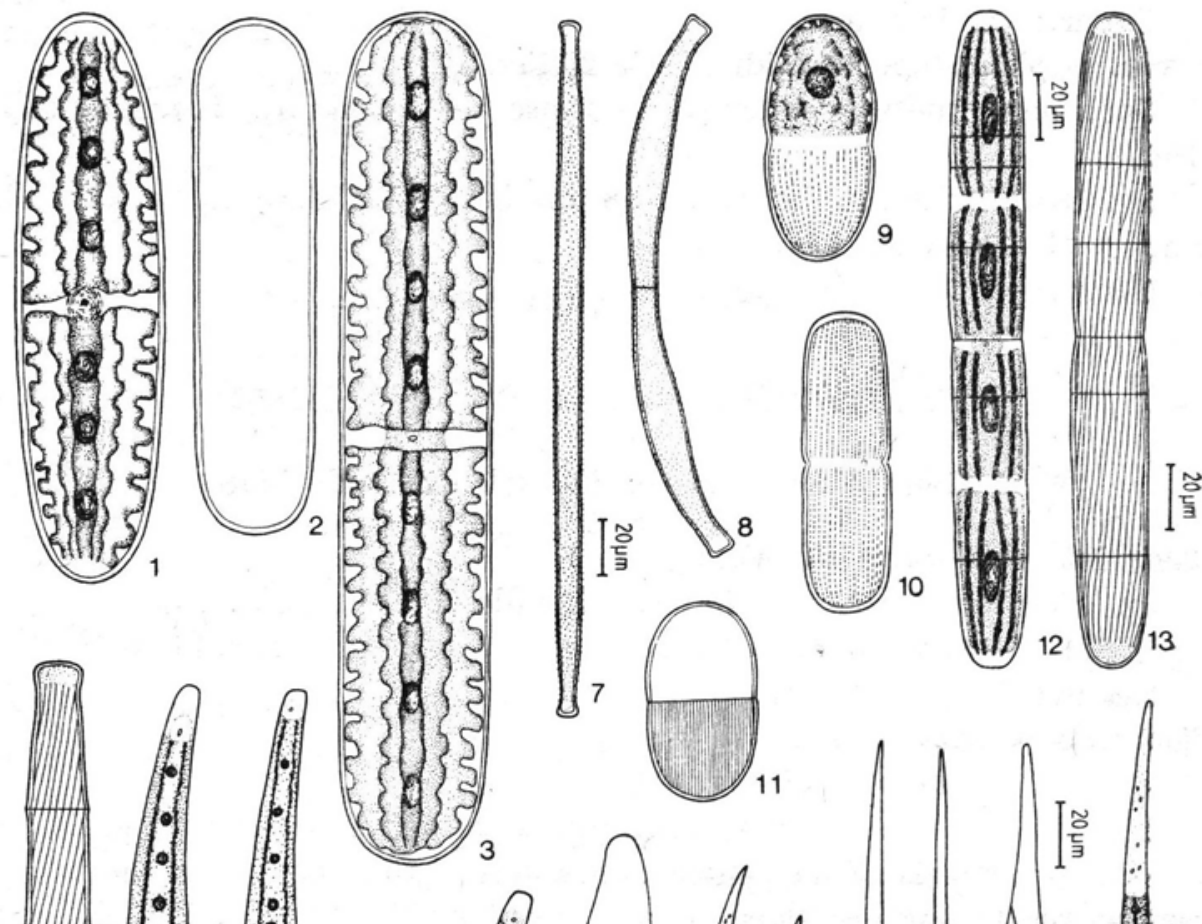

\section{5 \\ 3}
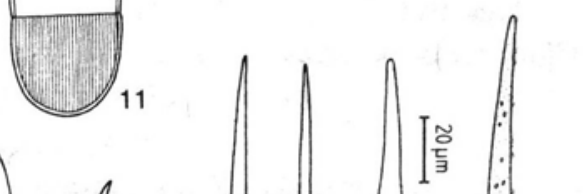

|VI.

101

[N $\left.18\right|_{0} ^{0}$

IN $\left.18\right|_{0} ^{0}$
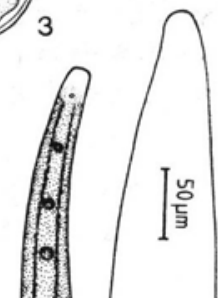

1

1.0.10 24

$\sqrt{25}_{26}$

(1)

1.

if 10 bed

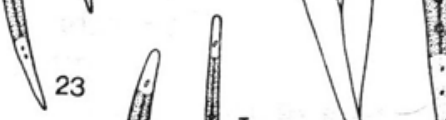

उईखु⿰

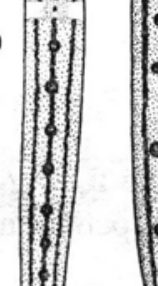

(30) 旅

(1)

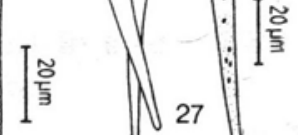

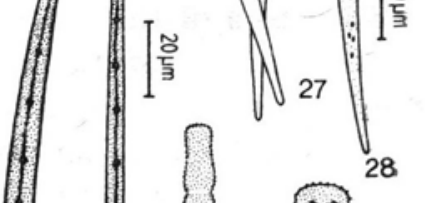

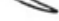

$20 \mu \mathrm{m}$ 
Cells straight, very narrow. In their dimensions they are close to some specimens of var. tenuius (Nordst.) W. Krieg., but differ from them in shape. Kossinskaya (1960) in taxon diagnosis stresses the fact that cells may be sometimes straight.

The specimens observed were classified as var. linea on the grounds of the cell shape - a differential feature between both taxa (var. tenuius and var. linea).

Lg. 87.4-88.5 $\mu \mathrm{m}$, br. $2.3 \mu \mathrm{m}$, ap. $\sim 1 \mu \mathrm{m}$, lg.: br. $38.00-38.48 \times$.

Occur.: 7rr.

Closterium angustatum Kütz. ex Ralfs

var. angustatum (Plate 5, Fig. 1-2)

Some specimens (Plate 5, Fig. 2) fit taxon var. gracile Kossinsk. $\mathrm{R}$ ůžička (1977) considers it as a synonym of the typical variety.

Lg. 282.2-581.0 $\mu \mathrm{m}$, br. 18.4-23.0 $\mu \mathrm{m}$, ap. 9.2-13.8 $\mu \mathrm{m}$, lg.: br. 14.03$-31.58 \times$, str. $2-3 / 10 \mu \mathrm{m}$.

Occur.: 2rr, 3rrr, 4rrr, 5rrr, 7rrr, 8rrr, 9rrr.

Closterium archerianum Cleve

var. archerianum (Plate 6, Fig. 4)

Lg. 224.1-294.6 $\mu \mathrm{m}$, br. 20.7-24.1 $\mu \mathrm{m}$, ap. 4.6-6.9 $\mu \mathrm{m}$, lg.: br. 9.74$-12.83 \times$, str. $5-6 / 10 \mu \mathrm{m}$.

Occur.: 7rrr, 9r.

Closterium baillyanum (Bréb.) Bréb.

var. baillyanum (Plate 4, Fig. 2)

Lg. 253.1-456.5 $\mu \mathrm{m}$, br. 27.6-50.6 $\mu \mathrm{m}$, ap. 16.1-18.4 $\mu \mathrm{m}$, lg.: br. 7.60-11.38 $\times$.

Occur.: 4rrr, 5rrr, 6rrr, 7rrr, 8rrr, 9rrr.

var. alpinum (Viret) Grönbl. (Plate 4, Fig. 1)

Lg. 381.8-556.1 $\mu \mathrm{m}$, br. 25.3-34.5 $\mu \mathrm{m}$, ap. 14.9-16.1 $\mu \mathrm{m}$, lg.: br. 12.77-18.64 $\times$.

Occur.: 2rrr, 7rrr, 8rrr, 9rrr.

\section{Plate 2}

Fig. 1. Netrium oblongum var. oblongum. Fig. 2. N. oblongum var. cylindricum. Fig. 3 . N. oblongum var. cylindricum, morpha. Fig. 4-5. Cylindrocystis brebissonii. Fig. 6 . C. crassa var. crassa. Fig. 7. Gonatozygon brebissonii var. brebissonii. Fig. 8. G. brebissonii var. kjellmanii. Fig. 9. Penium silvae-nigrae var. silvae-nigrae. Fig. 10. $P$. silvae-nigrae var. parallelum. Fig. 11. P. polymorphum var. polymorphum. Fig. 12-13. P. spirostriolatum var. spirostriolatum. Fig. 14. P. spirostriolatum var. amplificatum. Fig. 15. P. exiguum var. exiguum f. exiguum. Fig. 16. P. exiguum var. exiguum f. major. Fig. 17. P. exiguum var. capitatum. Fig. 18. Closterium tumidum var. tumidum. Fig. 19. Cl. tumidum, morpha 1. Fig. 20. Cl. tumidum, morpha 2. Fig. 21. Ol. toxon var. toxon, morpha. Fig. 22. Cl. lunula var. lunula. Fig. 23-24. Cl. acutum var. acutum. Fig. 25. Cl. acutum var. linea. Fig. 26. Ol. acutum var. linea, morpha. Fig. 27-28. Ol. idiosporum var. idiosporum. Fig. 29. Cl. exiguum var. exiguum. Fig. 30. Cl. navicula var. navicula. Fig. 31. Cl. cornu var. cornu. Fig. 32. Ol. cornu, morpha 
Closterium cornu Ehrenb. ex Ralfs

morpha (Plate 2, Fig. 32)

Cells nearly straight, only slightly curved at apices. Shape does not fit that of specimens of a typical variety (Plate 2, Fig. 31), whereas its breadth and length-breadth ratio especially do not correspond to those of var. cornu given by other authors: West, West (1904), Krieger (1933a, 1935), Taylor (1934), Hirano (1955), Bicudo (1969), Förster (1970), Islam (1970), Sarim, Faridi (1976), Parra, Gonzalez (1977), Oleksowicz (1978), Lind, Brook (1980) and others. Dimensions, proportions and partly a shape of cells are similar to those of $\mathrm{Cl}$. gracile Bréb. ex Ralfs, but they differ from them in a cell gradualy narrowed from median part towards apices. Also cell apices are not curved enough. Some characters correspond with those of individuals of Cl. cornu var. javanicum Gutw. Shape and dimensions nearly fit Hirano's (1972) description of this taxon. From Scott and Prescott's drawing (1961) it differs in the gentler narrowing towards apices and in dimensions (a cell is shorter and nearly 1.5 times narrower), whereas from Krieger's (1933a, 1935) drawing - in shape, as it is nearly straight, not curved.

Lg. $170.2 \mu \mathrm{m}$, br. $4.6 \mu \mathrm{m}$, ap. $2.3 \mu \mathrm{m}$, lg.: br. $37.00 \times$.

Occur.: 8rrr.

var. cornu (Plate 2, Fig. 31)

Lg. 121.9-209.3 $\mu \mathrm{m}$, br. 9.2-11.5 $\mu \mathrm{m}$, ap. 2.3-3.4 $\mu \mathrm{m}$, lg.: br. 13.25$18.20 \times$.

Occur.: 7rrr, 8rrr, 9rrr.

Closterium costatum Corda ex Ralfs

var. costatum (Plate 5, Fig. 3)

Lg. 265.6-365.2 $\mu \mathrm{m}$, br. 32.2-36.8 $\mu \mathrm{m}$, ap. 9.2-11.5 $\mu \mathrm{m}$, lg.: br. 7.82-9.98 $\times$, str. $2 / 10 \mu \mathrm{m}$.

Occur.: 4rrr, 7rrr, 8rrr, 9rr.

var. borgei (W. Krieg.) Růž. (Plate 5, Fig. 4)

Taxon var. borgei has been treated as a variety of $\mathrm{Cl}$. striolatum Ehrenb. ex Ralfs. so far. R užička (1972) has changed its taxonomical rank and classified it within $C l$. costatum species. I think Růžička's view to be justified. It is confirmed, especially by a lack of girdle bands in the specimens of this taxon. Although shape of cell apices (one of diagnostic characters of the genus) corresponds with that of $\mathrm{Cl}$. striolatum (angles rounded), but, as observed by R užička (1972), this character is highly variable. Angles may be from markedly protruded to reduced (rounded).

Lg. 340.3-365.2 $\mu \mathrm{m}$, br. $32.3 \mu \mathrm{m}$, ap. 9,2-10.3 $\mu \mathrm{m}$, lg.: br. 10.57-11.34 ×, str. $5 / 10 \mu \mathrm{m}$.

Occur.: 7rrr.

Closterium cynthia De Not.

var. oynthia (Plate 4, Fig. 12-13) 
a) Individuals typical. Cells strongly semicircularly curved (Plate 4, Fig. 12)

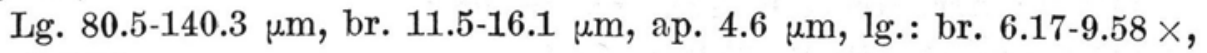
str. $6-7 / 10 \mu \mathrm{m}$.

Occur.: 7rrr, 9rrr.

b) morpha (Plate 4, Fig. 13)

Cells slender, less curved than typical. Medium part nearly cylindrical. Förster (1970), Růžička (1972, 1975a) and Coesel (1979a) describe similar cells.

Lg. 103.5-173.6 $\mu \mathrm{m}$, br. 10.3-14.9 $\mu \mathrm{m}$, ap. 3.4-4.6 $\mu \mathrm{m}$, lg.: br. 9.00$-11.80 \times$, str. $8-10 / 10 \mu \mathrm{m}$.

Occur.: 5rrr, 6rrr, 7rrr, 8rrr, 9rrr.

Closterium dianae Ehrenb. ex Ralfs

var. dianae (Plate 3, Fig. 10)

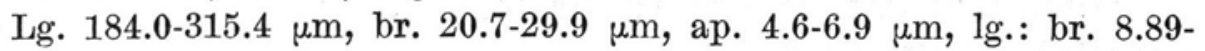
$11.97 \times$.

Occur.: 3rrr, 4rrr, 5rrr, 7rrr, 8rrr, 9rr.

var. pseudodianae (Roy) W. Krieg. (Plate 3, Fig. 11)

Lg. 170.2-230.0 $\mu \mathrm{m}$, br. 13.8-14.9 $\mu \mathrm{m}$, ap. 2.3-3.4 $\mu \mathrm{m}$, lg.: br. 12.33$16.67 \times$.

Occur.: 4rrr, 7rrr, 8rrr, 9rr.

Closterium directum Arch.

var. directum (Plate 6, Fig. 2)

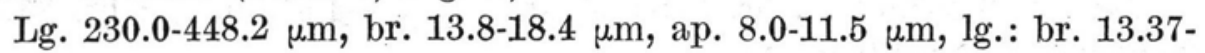
$26.81 \times$, str. $10-12 / 10 \mu \mathrm{m}$.

Occur.: 1rrr, 2e, 3e, 4ce, 5rr, 6rrr, 7rrr, 8ce, 9rrr.

Closterium exiguum W. et G. S. West

var. exiguum (Plate 2, Fig. 29)

Cell shape and dimensions fit description and drawings of $\mathrm{Cl}$. exiguum from Kossinskaya's work (1960).

Taxonomic rank of $\mathrm{Cl}$. exiguum W. et G. S. West 1902 has not been established yet. Krieger (1935) classifies it as a synonym of $\mathrm{Cl}$. parvulum Näg. var. angustum W. et G. S. West 1900, whereas specimens described by Roll (1915) as Cl. exiguum (drawings in Kossinskaya's, 1960 work) identifies as $\mathrm{Cl}$. acutum Bréb. var. variabile (Lemm.) W. Krieg.

Encountered by me specimens are similar in shape to $\mathrm{Cl}$. parvulum var. angustum, but differ from them in acute and not rounded cell spices. They are narrower and usually shorter. They fit in that semicircularly curved individuals of $\mathrm{Cl}$. acutum var. variabile. Therefore, specimens encountered may be classified as belonging to this taxon as well.

Lg. $62.1-92.0 \mu \mathrm{m}$, br. $2.3-4.6 \mu \mathrm{m}$, lg.: br. $14.50-27.00 \times$.

Occur.: 4rrr, 7rrr.

Closterium gracile Bréb. ex Ralfs

var. gracile (Plate 3, Fig. 6) 
Lg. 132.2-273.9 $\mu \mathrm{m}$, br. 4.6-8.0 $\mu \mathrm{m}$, ap. 2.3-3.4 $\mu \mathrm{m}$, lg.: br. 26.00$50.52 \times$.

Occur.: 2rrr, 4rr, 5rrr, 6rrr, 7rr, 8rr, 9r.

Closterium idiosporum W. et G. S. West

var. idiosporum (Plate 2, Fig. 27-28)

Mainly small specimens prevailed, corresponding to those described by Růžička (1973a).

Lg. 154.1-232.4 $\mu \mathrm{m}$, br. 8.0-11.5 $\mu \mathrm{m}$, ap. $2.3 \mu \mathrm{m}$, lg.: br. 16.75-20.41 $\times$, zygospore: lg. $42.5 \mu \mathrm{m}$, br. $26.4 \mu \mathrm{m}$.

Occur.: 1r, 2rr, 4r, 8rirr, 9rrr.

Closterium intermedium Ralfs

var. intermedium (Plate 5, Fig. 8)

Lg. 207.5-290.5 $\mu \mathrm{m}$, br. 16.1-19.5 $\mu \mathrm{m}$, ap. 5.7-10.3 $\mu \mathrm{m}$, lg.: br. 11.28$15.79 \times$, str. $5-6 / 10 \mu \mathrm{m}$.

Occur.: 2rrr, 3rrr, 4r, 5rrr, 6r, 7rr, 9rrr.

Closterium jenneri Ralfs

var. jenneri (Plate 3, Fig. 17)

Lg. 71.3-103.5 $\mu \mathrm{m}$, br. 10.3-13.8 $\mu \mathrm{m}$, ap. 3.4-4.6 $\mu \mathrm{m}$, lg.: br. 5.66-9.38 ×.

Occur.: 3rrr, 7ec, 8rrr, 9rrr.

Closterium juncidum Ralfs

var. juncidum (Plate 6, Fig. 1)

Cells in their median part more or less curved. They fit Förster's (1970) and Lenzenweger's (1970a) drawings.

Lg. 244.8-265.6 $\mu \mathrm{m}$, br. 9.2-11.5 $\mu \mathrm{m}$, ap. 3.4-4.6 $\mu \mathrm{m}$, lg.: br. 23.10$26.61 \times$, str. $10-12 / 10 \mu \mathrm{m}$.

Occur.: 7rrr.

Closterium kuetzingii Bréb.

var. kuetzingii (Plate 4, Fig. 6)

Lg. 323.7-522.9 $\mu \mathrm{m}$, br. 18.4-20.7 $\mu \mathrm{m}$, ap. 2.3-3.4 $\mu \mathrm{m}$, lg.: br. 16.44$28.42 \times$, str. $10-11 / 10 \mu \mathrm{m}$.

Occur.: 7rr, 8rrr, 9rrr.

Closterium leibleinii Kütz. ex Ralfs

var. leibleinii (Plate 3, Fig. 13-14)

a) Specimens correspond descriptions in the relevant literature (Plate 3, Fig. 13)

Lg. 115.0-156.4 $\mu \mathrm{m}$, br. 18.4-25.3 $\mu \mathrm{m}$, ap. 2.3-3.4 $\mu \mathrm{m}$, lg.: br. 5.89$7.56 \times$.

Occur.: 7rrr, 8rrr, 9rrr.

b) morpha (Plate 3, Fig. 14)

Cells less curved than typical, less inflated ventrally. Length-breadth ratio higher than cited in literature for typical specimens.

Lg. 138.0-151.8 $\mu \mathrm{m}$, br. 16.1-17.2 $\mu \mathrm{m}$, ap. 2.3-3.4 $\mu \mathrm{m}$, lg.: br. 8.49$9.43 \times$.

Occur.: 7rrr, 9rrr. 
Closterium limneticum Lemm.

var. tenue Lemm. (Plate 3, Fig. 7)

Lg. 197.8-207.0 $\mu \mathrm{m}$, br. 3.4-4,6 $\mu \mathrm{m}$, ap. $\sim 1 \mu \mathrm{m}$, lg.: br. $43.50-58.18 \times$. Occur.: 5rr.

Closterium lineatum Ehrenb. ex Ralfs

var. lineatum (Plate 3, Fig. 3-4)

Lg. 282.2-581.0 $\mu \mathrm{m}$, br. 16.1-20.7 $\mu \mathrm{m}$, ap. 4.6-9.2 $\mu \mathrm{m}$, lg.: br. 13.63$29.79 \times$, str. $7-8 / 10 \mu \mathrm{m}$.

Occur.: 2rrr, 4rr, 5rrr, 7rr, 9rrr.

Closterium lunula (Müll.) Nitzsch ex Ralfs

var. lunula (Plate 2, Fig. 22)

Lg. 439.9-581.0 $\mu \mathrm{m}$, br. 75.9-103.5 $\mu \mathrm{m}$, ap. 11.5-16.1 $\mu \mathrm{m}$, lg.: br. $5.36-6.69 \times$.

Occur.: 4rrr, 6rrr, 7rrr, 8rrr, 9rrr.

Closterium macilentum Bréb.

var. macilentum (Plate 6, Fig. 3)

Lg. 261.4-456.5 $\mu \mathrm{m}$, br. 11.5-18.4 $\mu \mathrm{m}$, ap. 4.6-5.7 $\mu \mathrm{m}$, lg.: br. 23.10$35.37 \times$.

Occur.: 4rrr, 7rrr, 8rrr, 9rrr.

Closterium moniliferum (Bory) Ehrenb. ex Ralfs

var. moniliferum (Plate 3, Fig. 2)

Lg. 186.3-232.4 $\mu \mathrm{m}$, br. 32.2-46.0 $\mu \mathrm{m}$, ap. 5.7-8.0 $\mu \mathrm{m}$, lg. : br. 4.20-6.79 $\times$.

Occur.: 7rr, 8rrr, 9rrr.

var. concavum Klebs (Plate 3, Fig. 1)

Lg. 197.9-240.7 $\mu \mathrm{m}$, br. 36.8-46.0 $\mu \mathrm{m}$, ap. 5.7-6.9 $\mu \mathrm{m}$, lg. : br. 5.11-5.38 $\times$.

Occur.: 7 rrr.

Closterium navicula (Bréb.) Lütkem.

var. navicula (Plate 2, Fig. 30)

Lg. 31.9-69.0 $\mu \mathrm{m}$, br. 9.2-16.1 $\mu \mathrm{m}$, ap. 4.6-6.9 $\mu \mathrm{m}$, lg.: br. 3.00-5.00 $\times$.

Occur.: 2rrr, 4rrr, 7rrr, 8rr, 9rr.

Closterium parvulum Näg.

var. parvulum f. parvulum (Plate 3, Fig. 15)

Lg. 105.8-156.4 $\mu \mathrm{m}$, br. 10.3-16.1 $\mu \mathrm{m}$, ap. 2.3-3.4 $\mu \mathrm{m}$, lg.: br. 8.43$12.60 \times$.

Occur.: 2rrr, 4r, 7rr, 8ce, 9rr.

f. maius Schmidle (Plate 3, Fig. 16)

According to Růžička (1973a), authorship of the form I confine to Schmidle and not to W. West as Kossinskaya (1960), Förster (1970) and others do.

Individuals of this form were found only in the water body which totally differed from others in habitat. In others only specimens of $f$. parvulum were encountered. Probably, ecological conditions of this water body determined a development of the first form. 


\section{PLATE 3}

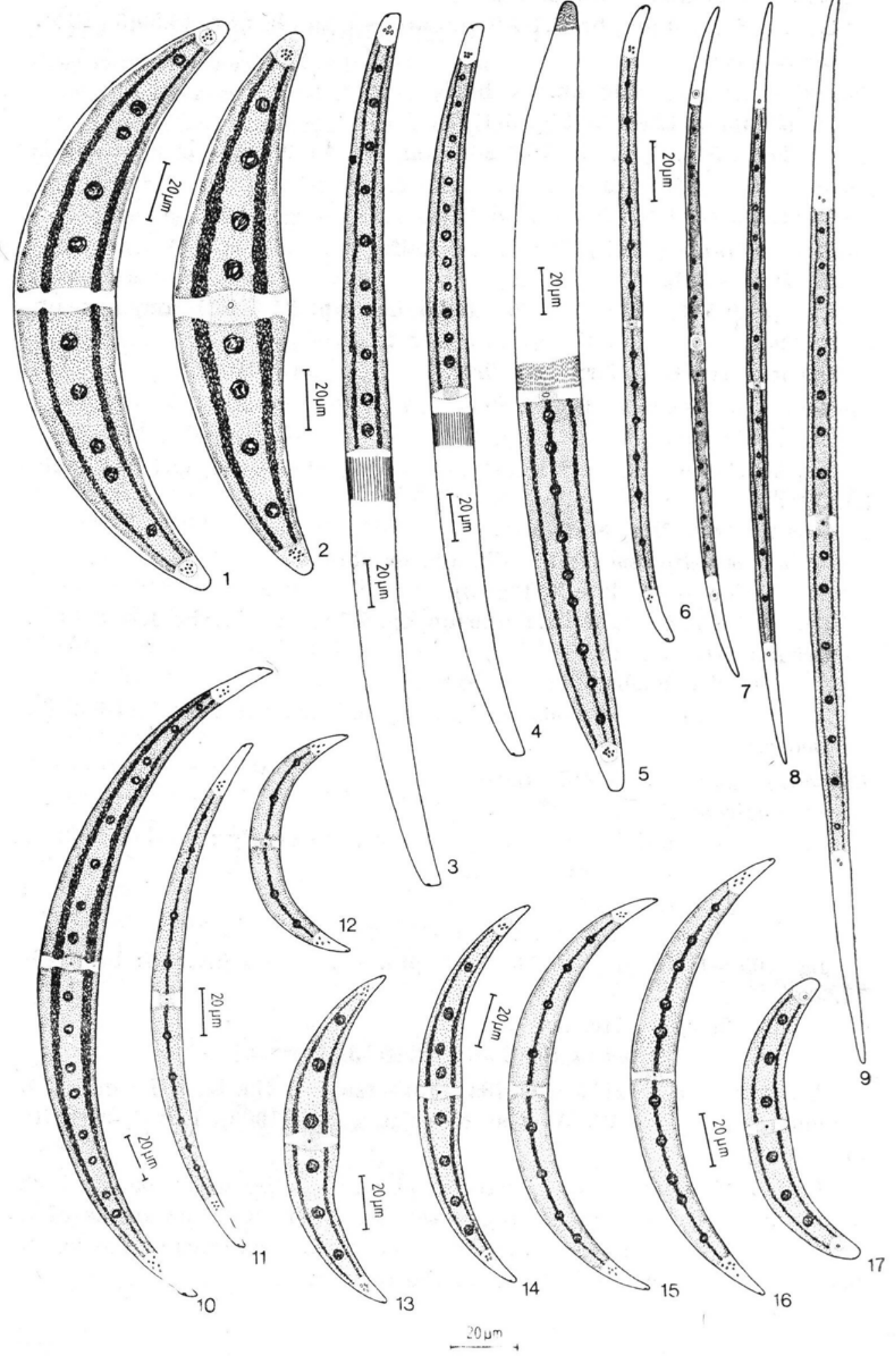




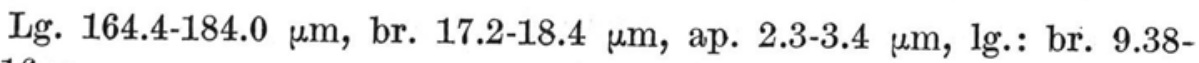
$10.16 \times$.

Occur.: 7rrr.

Closterium praelongum Bréb.

var. praelongum (Plate 4, Fig. 8-9)

Amongst encountered individuals two groups which differ in cell shape and dimensions can be distinguished:

a) cells narrow, slender, dorsal margin convex, ventral margin concave. They fit a description of typical individuals (Plate 4, Fig. 8).

Lg. 348.6-485.5 $\mu \mathrm{m}$, br. $11.5 \mu \mathrm{m}$, ap. 3.4-4.6 $\mu \mathrm{m}$, lg.: br. 30.31$42.22 \times$, str. $8 / 10 \mu \mathrm{m}$,

Occur.: $7 \mathrm{rr}$.

b) morpha (Plate 4, Fig. 9)

Cells long, thick, margins parallel at considerable length. Length-breadth ratio of some specimens smaller than that according to literature: West, West (1904), Krieger (1935), Hirano (1955), Kossinskaya (1960), Förster (1970), Růžička (1973a). It rather corresponds with that given for specimens of var. brevius. On the other hand, their length considerably exceeds upper limit of this parameter given for the taxon mentioned above by: West, West (1904), Krieger (1935), Rủžička (1955, 1957a, 1973a, 1977), Förster (1970), and Sarim, Faridi (1976). If cell length, and not length-breadth ratio, is taken into account as a differentiating character between two taxons, (var. praelongum and var. brevius), present specimens should be classified as belonging to the typical variety.

Lg. 481.4-564.4 $\mu \mathrm{m}$, br. 20.7-25.3 $\mu \mathrm{m}$, ap. 4.6-5.7 $\mu \mathrm{m}$, lg.: br. 19.98$26.46 \times$.

Occur.: $7 \mathrm{rrr}$.

var. brevius (Nordst.) W. Krieg. (Plate 4, Fig. 7)

Lg. 174.8-286.3 $\mu \mathrm{m}$, br, 11.5-12.6 $\mu \mathrm{m}$, ap. $3.4 \mu \mathrm{m}$, lg.: br. 15.20$22.72 \times$.

Occur.: 7rr.

Specimens identified by many as var. brevius (Nordst.) W Krieg. totally differ in shape from the holotype of f. brevius Nordst. 1888, draw-

\section{Plate 3}

Fig. 1. Closterium moniliferum var. concavum. Fig. 2. Cl. moniliferum var. moniliferum. Fig. 3-4. Cl. lineatum var. lineatum. Fig. 5. Cl. pritchardianum var. pritchardianum. Fig. 6. Ol. gracile var. gracile. Fig. 7. Cl. limneticum var. tenue. Fig. 8. Cl. pronum var. pronum f. brevius. Fig. 9. Ol. pronum var. pronum f. pronum. Fig. 10. Cl. dianae var. dianae. Fig. 11. Ol. dianae var. pseudodianae. Fig. 12. Ol. venus var. venus. Fig. 13. Ol. leibleinii var. leibleinii. Fig. 14. Cl. leibleinii var. leibleinii, morpha. Fig. 15. Cl. parvulum var. parvulum f. parvulum. Fig. 16. Cl. parvulum var. parvulum f. maius.

Fig. 17. Ol. jenneri var. jenneri 


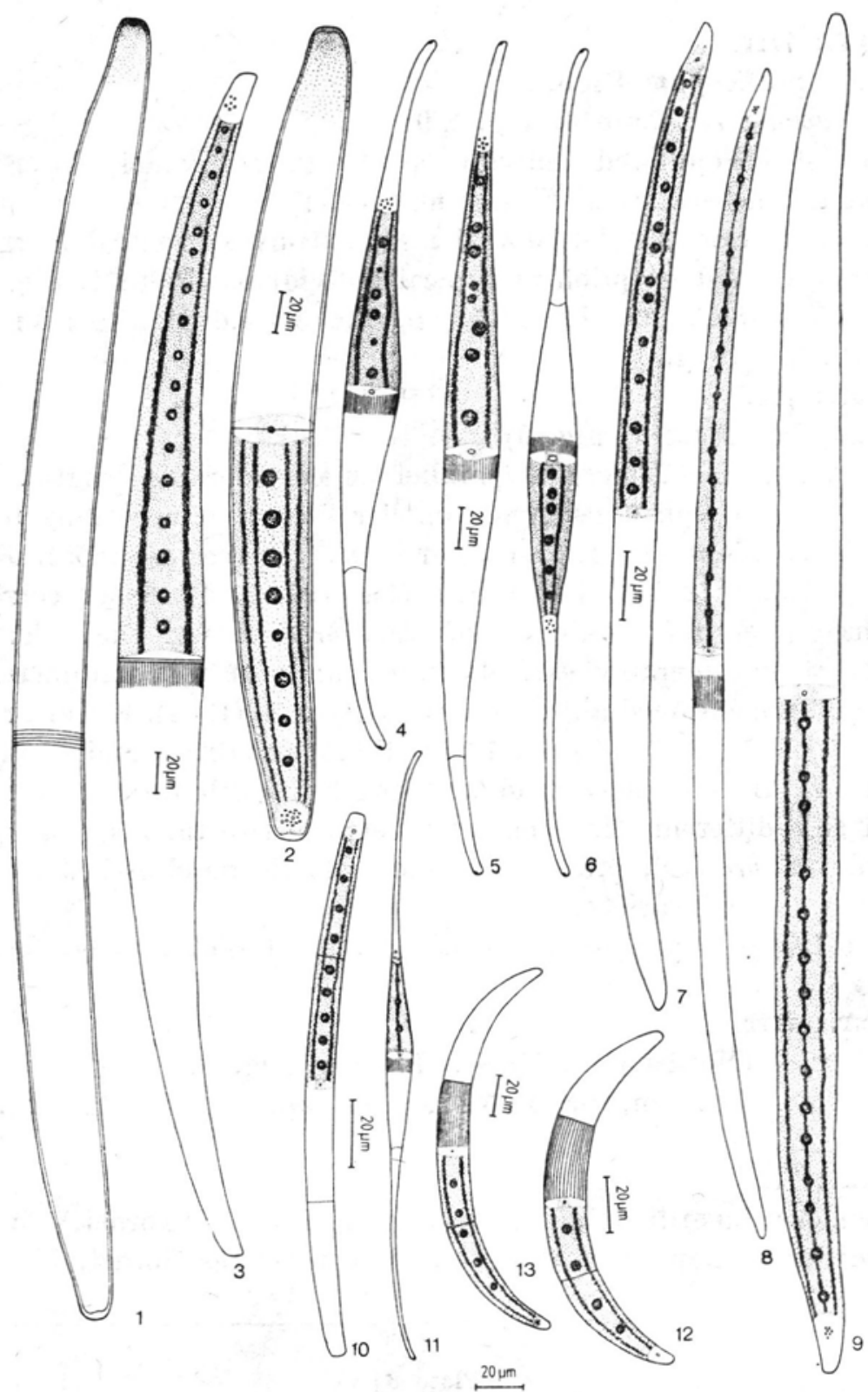

Fig. 1. Olosterium baillyanum var. alpinum. Fig. 2. Ol. baillyanum var. baillyanum. Fig. 3. Cl. ralfsii var. hybridum. Fig. 4. Cl. rostratum var. rostratum f. rostratum. Fig. 5. Cl. rostratum var. rostratum f. brevirostratum. Fig. 6. Cl. kuetzingii var. kuetzingii. Fig. 7. Cl. praelongum var. brevius. Fig. 8. Cl. praelongum var. praelongum. Fig. 9. Cl. praelongum var. praelongum, morpha. Fig. 10. Cl. abruptum var. abruptum f. angustissimum. Fig. 11. Cl. setaceum var. setaceum, morpha. Fig. 12. Cl. cynthia var. cynthia. Fig. 13. Cl. cynthia var. cynthia, morpha 
ing in the Růžička's work (1955). They rather correspond with specimens of var. praelongum and they differ from them merely in dimensions. It seems that taxonomy of $\mathrm{Cl}$. praelongum Bréb. should be slightly corrected. And individuals (according to Rưžička (1955) in addition to Nordstedt's diagnosis) with dorsal margin uniformly curved and ventral margin straight or slightly convex should belong to var. brevius (Nordst.) W. Krieg. Those specimens are usually small. Whereas, small individuals with parallel margins in the midregion, or dorsal margin convex and ventral margin concave should belong to var. praelongum with the widen taxon variation in dimensions. If any differences in ecological requirements are discovered between small and large specimens, the latter ought to be treated as var. praelongum f. brevius (in a new and not Nordstedt's sense).

Closterium pritchardianum Arch.

var. pritchardianum (Plate 3, Fig. 5)

Lg. 315.4-336.1 $\mu \mathrm{m}$, br. 27.6 $\mu \mathrm{m}$, ap, 6.9-8.0 $\mu \mathrm{m}$, lg.: br. 11.42-12.18 $\times$, str. $12 / 10 \mu \mathrm{m}$.

Occur.: 7rrr.

Closterium pronum Bréb.

var. pronum f. pronum (Plate 3, Fig. 9)

Lg. 298.8-385.9 $\mu \mathrm{m}$, br. 6.9-9.2 $\mu \mathrm{m}$, ap. $2.3 \mu \mathrm{m}$, lg.: br. 33.38-48.12 $\times$. Occur.: 1rrr, 2rrr, 3rrr, 4r, 5rr, 6rrr, 7rrr, 8rrr, 9rr.

f. brevius W. West (Plate 3, Fig. 8)

Lg. 186.3-290.5 $\mu \mathrm{m}$, br. 5.7-9.2 $\mu \mathrm{m}$, ap. 1.1-2.3 $\mu \mathrm{m}$, lg.: br. 22.25$39.74 \times$.

Occur.: 1r, 2rr, 3cc, 4cc, 5c, 6cc, 7rrr, 8ce, 9c.

Both forms are similar in shape, but differ in their dimensions. Krieger (1935) and Růžička (1977) treat them as one taxon.

In the present paper both forms have been distinguished. On the grounds of my observations they seem to occupy rather distinct ecological niches. Specimens of $\mathrm{f}$. brevius were more often found in samples than those of. f. pronum. Cells of f. brevius occured in large numbers mainly in plankton, while those of f. pronum - in squeezes from shallow basins and excavation margins.

Sometimes it was quite difficult to classify a specimen to one of these forms. Then cell length was assumed as a criterion, as cells did not differ in breadth.

Closterium ralfsii Bréb. ex Bréb.

var. hybridum Rabenh. (Plate 4, Fig. 3)

Lg. 340.3-572.7 $\mu \mathrm{m}$, br. 29.9-41.4 $\mu \mathrm{m}$, ap. 6.9-9.2 $\mu \mathrm{m}$, lg.: br. 9.86$18.32 \times$, str. $7-9 / 10 \mu \mathrm{m}$.

Occur.: 7rrr.

Closterium rostratum Ehrenb. ex Ralfs

var. rostratum f. rostratum (Plate 4, Fig. 4) 
Lg. 282.2-385.9 $\mu \mathrm{m}$, br. 18.4-25.3 $\mu \mathrm{m}$, ap. 3.4-4.6 $\mu \mathrm{m}$, lg.: br. 12.81$15.34 \times$, str. $10 / 10 \mu \mathrm{m}$.

Occur.: 7rr.

f. brevirostratum (W. West) Kossinsk. (Plate 4, Fig. 5)

Cell dimensions correspond with those of f. rostratum, whereas short apical processes indicate f. brevirostratum as the proper one.

Krieger (1935) considers this taxon as a synonym of f. rostratum, while Kossinskaya (1960) and Růžička (1977) distinguish it. Kossinskaya stresses the differences between this taxon and typical form, though some intermediate forms may be also found.

Lg. $402.5 \mu \mathrm{m}$, br. $26.4 \mu \mathrm{m}$, ap. $4.6 \mu \mathrm{m}$, lg.: br. $15.25 \times$, str. $8 / 10 \mu \mathrm{m}$.

Occur.: 7rrr.

Closterium setaceum Ehrenb. ex Ralfs

var. setaceum, morpha (Plate 4, Fig. 11)

Specimens differ in shape from those described by: Migula (1907), Krieger (1935), Hirano (1955), Ito (1974), R užička (1977) and Coesel (1979a). Cell ventral margin is more convex than dorsal, midregion is elongated and forms $1 / 3$ of the cell length. Similar drawings of this taxon may be found in works of: Kossinskaya (1960), Grönblad et al. (1968), Förster (1970) and others. Specimens described by Grönblad et al. (1968) have got smooth cell wall.

Lg. 207.5-307.1 $\mu \mathrm{m}$, br. 9.2-11.5 $\mu \mathrm{m}$, ap. 1.1-2.3 $\mu \mathrm{m}$, lg.: br. 23.10$30.67 \times$, str. $11-12 / 10 \mu \mathrm{m}$.

Occur.: 4rr, 5rrr, 6rrr, 7rrr, 8rrr, 9r. Closterium striolatum Ehrenb. ex Ralfs

var. striolatum (Plate 5, Fig. 5-6)

a) Cells stout, dorsal margin markedly convex, they fit description, of typical individuals (Plate 5, Fig. 5).

Lg. 219.9-365.2 $\mu \mathrm{m}$, br. 26.4-39.1 $\mu \mathrm{m}$, ap. 10.3-12.6 $\mu \mathrm{m}$, lg.: br. 7.23$11.94 \times$, str. 6-7/10 $\mu \mathrm{m}$.

Occur.: 1rrr, 2ce, 3ec, 4c, 5c, 6c, 7rrr, 8rr, 9rr.

b) morpha (Plate 5, Fig. 6)

Cells slender, slightly resemble cells of $\mathrm{Cl}$. striolatum var. erectum Klebs, but differ from them in slightly convex dorsal margin and less curved apices.

Plate 5

Fig. 1-2. Closterium angustatum var. angustatum. Fig. 3. Cl. costatum var. costatum. Fig. 4. Cl. costatum var. borgei. Fig. 5. Cl. striolatum var. striolatum. Fig. 6. Cl. striolatum var. striolatum, morpha. Fig. 7. Cl. striolatum var. erectum. Fig. 8. Cl. intermedium var. intermedium. Fig. 9. Pleurotaenium tridentulum var. tridentulum. Fig. 10. Pl. trabecula var. trabecula. Fig. 11. Pl. trabecula var. crassum. Fig. 12-14. Pl. mimutum var. minutum f. minutum. Fig. 15. Pl. minutum var. latum 
PLATE 5

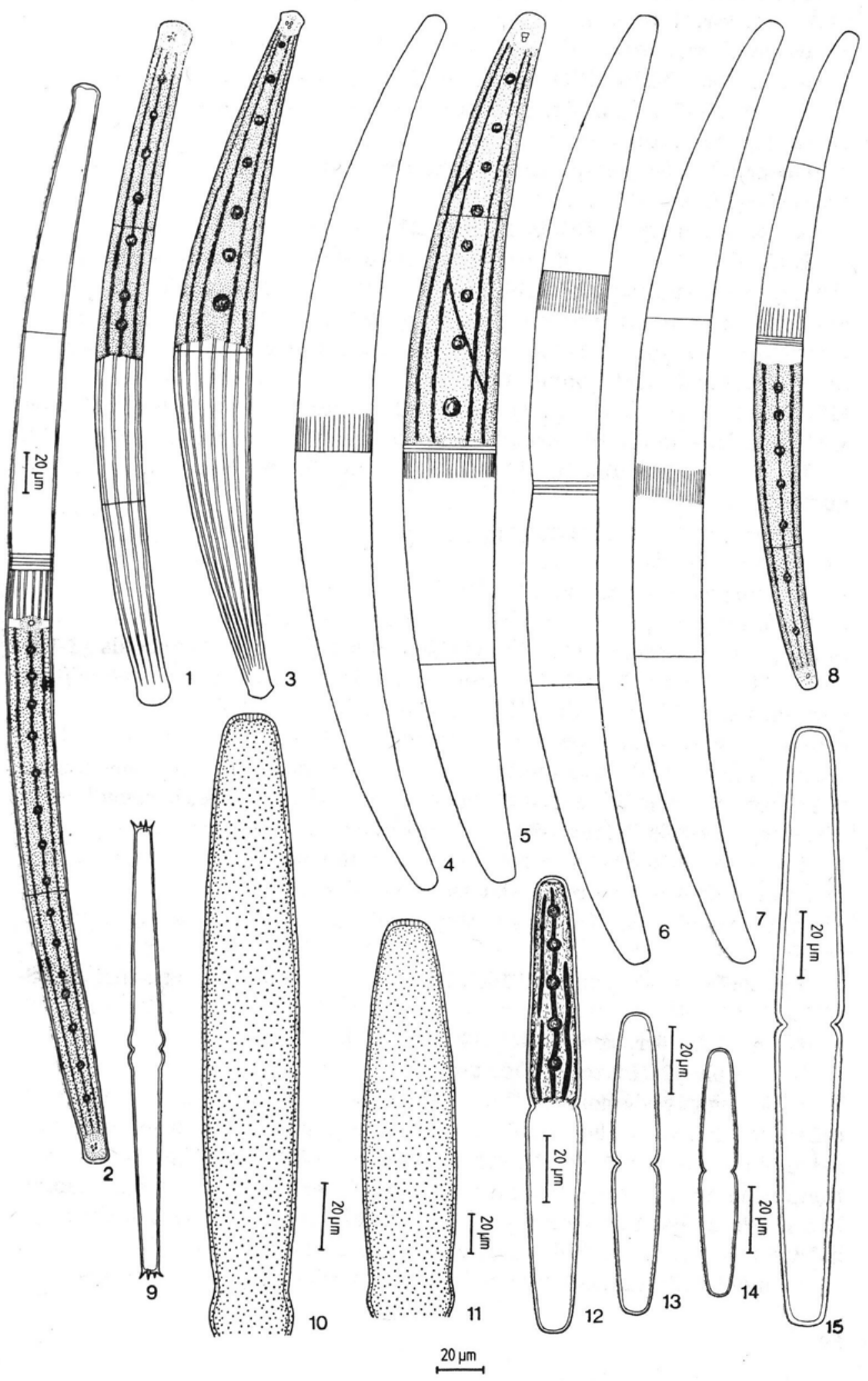




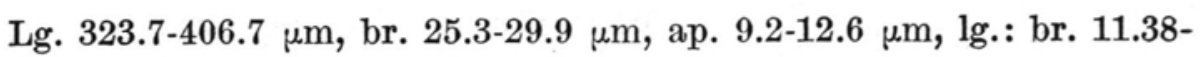
$14.58 \times$, str. $7 / 10 \mu \mathrm{m}$.

Occur.: 5rrr, 6rrr.

var. erectum Klebs (Plate 5, Fig. 7)

Lg. 286.3-473.1 $\mu \mathrm{m}$, br. 23.0-31.0 $\mu \mathrm{m}$, ap. 9.2-11.5 $\mu \mathrm{m}$, lg.: br. 11.10$15.82 \times$, str. $7 / 10 \mu \mathrm{m}$.

Occur.: 2c, 3ce, 4rr, 5c, 6c, 7rrr, 8rrr, 9rrr.

Closterium toxon W. West

var. toxon, morpha (Plate 2, Fig. 21)

Individuals slightly differ from those described by: West, West (1904), Kossinskaya (1960), Förster (1970), Lind, Brook (1980) and others. Cells margins are parallel at rather short distance, then narrowed towards apices. Some specimens are broader than those described in papers mentioned above. They are similar to drawing in Krieger's (1935) work (Plate 23, Fig. 4). They only differ in the length of cell part with parallel sides, it was usually very short in the specimens encountered.

Lg. 232.4-298.8 $\mu \mathrm{m}$, br. 11.5-19.5 $\mu \mathrm{m}$, ap. 5.7-6.9 $\mu \mathrm{m}$, lg.: br. 14.05$20.21 \times$.

Occur.: 5rrr, 6rrr, 8rrr, 9rrr.

Closterium tumidum Johns.

a) morpha 1 (Plate 2, Fig. 19)

Cells longer than those of var. tumidum, they also have got broader apices, are less tumid in the midregion and have more pyrenoids (8-10). They fit Förster's (1970) drawing of $\mathrm{Cl}$. tumidum var. nylandicum Grönbl. form (Plate 2, Fig. 17). If compared to the holotype var. nylandicum Grönbl. 1921, specimens are longer, broader for the whole cell length, more gently attenuated from median part towards apices, cell pole shorter (from the chromatophore end to the cell apex), vacuole with vibrating crystals is located nearer cell apex.

From the differences mentioned above it may be inferred that specimens described are more similar to those of var. tumidum, than to those of var. nylandicum. Therefore, they probably represent a form of this taxon.

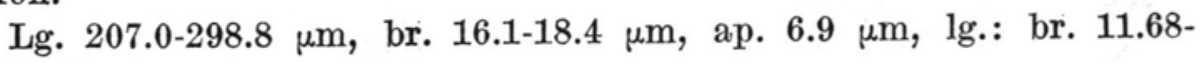
$18.56 \times$.

Occur.: 2r, 6rr, 8rrr.

b) morpha 2 (Plate 2, Fig. 20)

Cells slightly sigmoid at the considerable cell length. This is the only difference between them and those of morpha 1 . They also somewhat resemble encountered specimens of $C l$. toxon $\mathrm{W}$. West. They differ from them in more in curved median part, straight cell apices, and larger number of pyrenoids. Förster (1970, Plate 2, Fig. 16) has described the same individuals and classified them as Cl. tumidum var. nylandicum, form.

Sigmoid cells are not rare within genus Closterium. Such forms have 
been encountered, among others, in? Cl. venus Kütz. ex Ralfs (Wołoszyńska 1914), Cl. acerosum (Schr.) Ehrenb. ex Ralfs and Cl. leibleinii Kütz. ex Ralfs (Deflandre 1925), Cl. littorale Gay (Růžička 1973b). Lg. $249.0 \mu \mathrm{m}$, br. $18.4 \mu \mathrm{m}$, ap. $6.9 \mu \mathrm{m}$, lg.: br. $13.53 \times$.

Occur.: 2rrr.

var. tumidum (Plate 2, Fig. 18)

Lg. 115.0-171.3 $\mu \mathrm{m}$, br. 12.6-19.5 $\mu \mathrm{m}$, ap. 4.6-5.7 $\mu \mathrm{m}$, lg.: br. 7.70$10.29 \times$.

Occur.: 1rrr, 2rr, 3rrr, 4r, 5rrr, 6rrr, 8rr, 9rrr.

Closterium venus Kütz. ex Ralfs

var. venus (Plate 3, Fig. 12)

Lg. 48.3-85.1 $\mu \mathrm{m}$, br. 6.9-11.5 $\mu \mathrm{m}$, ap. $2.3 \mu \mathrm{m}$, lg.: br. 5.50-9.25 $\times$. Occur.: 2rrr, 4rrr, 5rrr, 7e, 8rrr, 9c.

\section{Suborder：DESMIDIINEAE}

Family: Desmidiaceae Ralfs 1848

Genus: Pleurotaenium Nägeli 1849

Pleurotaenium ehrenbergii (Bréb.) De Bary

var. ehrenbergii (Plate 6, Fig. 5)

Lg. 307.1-527.0 $\mu \mathrm{m}$, br. max. 25.3-31.0 $\mu \mathrm{m}$, isth. 21.8-25.3 $\mu \mathrm{m}$, ap. $18.4 \mu \mathrm{m}$, lg.: br. $11.13-18.70 \times$.

Occur.: 7rr.

Pleurotaenium minutum (Ralfs) Delp.

var. minutum f. minutum (Plate 5, Fig. 12-14)

Cell dimensions and proportions are main criteria in distinguishing. infraspecific taxa within $\mathrm{Pl}$. minutum. It has resulted in many small taxa which are sometimes very difficult to identify.

When mentioned-above criteria are taken into account, following forms may be distinguished in the material studied: typical individuals (Plate 5, Fig. 12), small individuals (Plate 5, Fig. 14) - according to those described f. minus (Racib.) Kossinsk., and individuals (Plate 5, Fig. 13) "intermediate" between these taxa. I think that all of them belong to one taxon only i.e. f. minutum, therefore, f. minus should be liquidated.

Lg. 73.6-167.9 $\mu \mathrm{m}$, br. $\max$. 10.3-17.2 $\mu \mathrm{m}$, isth. 9.2-16.1 $\mu \mathrm{m}$, ap. 6.9-11.5 $\mu \mathrm{m}$, lg.: br. 6.40-12.00 $\times$.

Occur.: 1rrr, 2rrr, 3rr, 4rr, 5rrr, 7rrr, 8rrr, 9rrr.

var. latum Kaiser (Plate 5, Fig. 15)

Lg. $172.5 \mu \mathrm{m}$, br. $\max .19 .5 \mu \mathrm{m}$, isth. $14.9 \mu \mathrm{m}$, ap. $11.5 \mu \mathrm{m}$, lg.: br. $8.85 \times$.

Occur.: 3rrr. 
Plerotaenium rectum Delp.

var. rectum (Plate 6, Fig. 6-7)

Lg. 232.4-324.5 $\mu \mathrm{m}$, br. $\max$ 18.4-20.7 $\mu \mathrm{m}$, isth. 12.6-18.4 $\mu \mathrm{m}$, ap. 12.6-16.1 $\mu \mathrm{m}$, lg.: br. 11.23-17.64 $\times$.

Occur.: 1rrr, 2rrr, 3rr, 4rrr, 7rrr, 9rrr.

Plerotaenium trabecula Ehrenb. ex Näg.

var. trabecula (Plate 5, Fig. 10)

Lg. 276.0-373.5 $\mu \mathrm{m}$, br. $\max .25 .3-32.2 \mu \mathrm{m}$, isth. 20.7-25.3 $\mu \mathrm{m}$, ap. 16.1-18.4 $\mu \mathrm{m}$, lg.: br. 8.57-13.01 $\times$.

Occur.: 4rrr, 7r, 8rrr, 9rrr.

var. crassum Wittr. (Plate 5, Fig. 11)

Lg. 373.5-432.4 $\mu \mathrm{m}$, br. $\max$. 48.3-50.6 $\mu \mathrm{m}$, isth. 39.1-43.7 $\mu \mathrm{m}$, ap. 23.0-25.3 $\mu \mathrm{m}$, lg.: br. 7.38-8.95 $\times$.

Occur.: 4rrr, 7rrr.

Pleurotaenium tridentulum (Wolle) W. West

var. tridentulum (Plate 5, Fig. 9)

Lg. 192.0-209.3 $\mu \mathrm{m}$, br. $\max$. 14.9-16.1 $\mu \mathrm{m}$, isth. 12.6-13.8 $\mu \mathrm{m}$, ap. 6.9-8.0 $\mu \mathrm{m}$, lg.: br. 12.40-14.05 $\times$.

Occur.: 1rrr, 4ri, 9rrr.

\section{Genus: Tetmemorus Ralfs ex Ralfs 1848}

Tetmemorus brebissonii (Menegh.) Ralfs ex Ralfs

var. brebissonii (Plate 6, Fig. 9)

Lg. 105.8-138.0 $\mu \mathrm{m}$, br. max. 20.7-25.3 $\mu \mathrm{m}$, isth. 16.1-18.4 $\mu \mathrm{m}$, ap. 16.1-20.7 $\mu \mathrm{m}$, lg.: br. $4.60-6.00 \times$.

Occur.: 1rr, 2rrr, 3rrr, 4r, 5rrr, 6rrr, 7rrr, 9rr.

var. minor De Bary (Plate 6, Fig. 8)

Lg. 67.8-97.7 $\mu \mathrm{m}$, br. max. 16.1-21.8 $\mu \mathrm{m}$, isth. 13.8-18.4 $\mu \mathrm{m}$, ap. 16.1-21.8 $\mu \mathrm{m}, \mathrm{lg} .:$ br. $3.50-4.48 \times$.

Occur.: 1rrr, 2rrr, 3rrr, 4rrr, 6rrr.

Tetmemorus granulatus (Bréb.) Ralfs ex Ralfs

var. granulatus (Plate 6, Fig. 15-17)

In the study area two groups of individuals which differ in dimensions may be distinguished within this taxon:

a) Individuals of average size (Plate 6, Fig. 15)

Lg. 115.0-133.4 $\mu \mathrm{m}$, br. $\max$. 25.3-28.7 $\mu \mathrm{m}$, isth. 21.8-26.4 $\mu \mathrm{m}$, ap. 11.5-16.1 $\mu \mathrm{m}, \mathrm{lg} .:$ br. 4.00-5.18 $\times$.

Occur.: 1rrr, 2rrr, 3rr, 4rr, 5rrr, 8rrr, 9rrr.

A specimen with abnormally formed apex of one semicell - teratologic form was encountered (Plate 6, Fig. 16)

b) large individuals (Plate 6, Fig. 17) 
Lg. 207.0-240.7 $\mu \mathrm{m}$, br. max. 41.4-47.1 $\mu \mathrm{m}$, isth. 39.1-41.4 $\mu \mathrm{m}$, ap. 20.7-23.0 $\mu \mathrm{m}$, lg.: br. 5.00-5.11 $\times$.

Occur.: 3rrr.

var. attenuatus W. West (Plate 6, Fig. 13-14)

In two different sites specimens of different size were found:

a) morpha 1 (Plate 6, Fig. 13)

Cells small, their dimensions oscillate around the lower limit noted for the individuals of var. attenuatus.

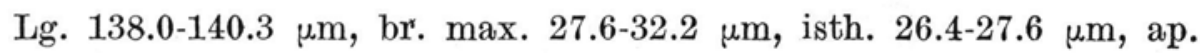
13.8-17.2 $\mu \mathrm{m}$, lg.: br. 4.36-5.00 $\times$.

Occur.: 5rrr.

b) morpha 2 (Plate 6, Fig. 14)

Cells big, slightly wider than those described in the relevant literature.

Lg. 262.2-265.6 $\mu \mathrm{m}$, br. max. 50.6-51.7 $\mu \mathrm{m}$, isth. 41.4-43.7 $\mu \mathrm{m}$, ap. $19.5 \mu \mathrm{m}$, lg.: br. 5.14-5.18 $\times$.

Occur.: 3rrr.

Tetmemorus laevis Kütz. ex Ralfs

var. laevis (Plate 6, Fig. 10)

Lg. 64.4-80.5 $\mu \mathrm{m}$, br. $\max$. 17.2-21.8 $\mu \mathrm{m}$, isth. 14.9-20.7 $\mu \mathrm{m}$, ap.

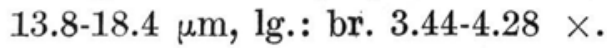

Occur.: 1rrr, 2rrr, 3rrr, 4rr, 5rr, 6rrr, 9rrr.

var. indermedius (Voron.) Rủž. (Plate 6, Fig. 11)

Lg. 58.6-69.0 $\mu \mathrm{m}$, br. max. $19.5 \mu \mathrm{m}$, isth. $18.4 \mu \mathrm{m}$, ap. $16.1 \mu \mathrm{m}$, lg.: br. 3.01-3.54 $\times$.

Occur.: 1rrr, 9rrr.

var. minutus (De Bary) W. Krieg. (Plate 6, Fig. 12)

Lg. 55.2-69.0 $\mu \mathrm{m}$, br. max. 18.4-20.7 $\mu \mathrm{m}$, isth. 17.2-18.4 $\mu \mathrm{m}$, ap. 10.3-13.8 $\mu \mathrm{m}$, lg.: br. $3.00-3.33 \times$.

Occur.: 3rrr, 4rrr.

Systematic affiliation of both taxa of Tetmemorus laevis (var. intermedius and var. minutus) has not been, hitherto, elucidated. Firstly, they were treated as units of the species rank, next they were included in Tetmemorus laevis as varieties. Recently, Förster (1970) has considered them as synonymes of var. laevis. He believes that differences between them fall within the variation limits of the typical variety. Simultaneously, he reminds according to Rủžička (1959) differences between these taxa, although distinguished by the latter, and var. laevis are of little taxonomic value.

In this paper I have decided to distinguish both taxa within var. laevis, as distinct morphological differences between representatives of these three taxa enable to identify them easily (a size of individuals, a cell shape, a number of pyrenoids, cell side view). In the material studied I have not found any intermediate forms. 


\section{PLATE 6}

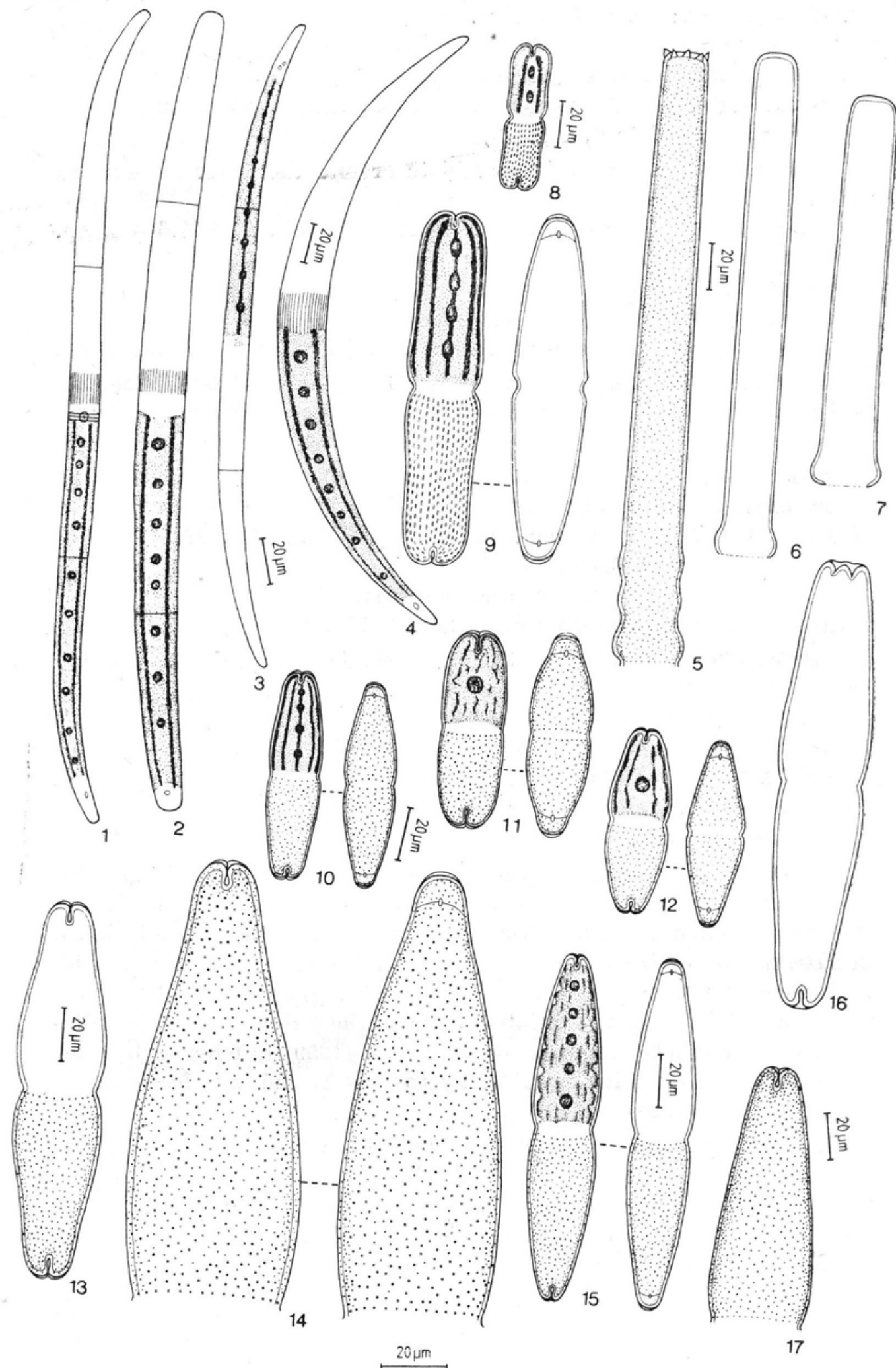


Euastrum affine Ralfs ex Ralfs

var. affine (Plate 7, Fig. 1)

Lg. 105.8-117.3 $\mu \mathrm{m}$, br. 55.2-66.7 $\mu \mathrm{m}$, isth. 16.1-17.2 $\mu \mathrm{m}$, lob. pol. br. 25.3-34.5 $\mu \mathrm{m}$, th. $41.4 \mu \mathrm{m}$, lg.: br. 1.76-2.00 $\times$.

Occur.: 2rrr, 3rrr, 4rr.

Euastrum ampullaceum Ralfs

var. ampullaceum (Plate 7, Fig. 2)

Lg. 87.4-98.9 $\mu \mathrm{m}$, br. 50.6-59.8 $\mu \mathrm{m}$, isth. 16.1-20.7 $\mu \mathrm{m}$, lob. pol. br. 23.0-29.9 $\mu \mathrm{m}$, th. $34.5 \mu \mathrm{m}$, lg.: br. 1.65-1.75 $\times$.

Occur.: 3rrr.

Euastrum ansatum Ehrenb. ex Ralfs

var. ansatum (Plate 7, Fig. 3)

Lg. 80.5-96.6 $\mu \mathrm{m}$, br. 42.5-48.3 $\mu \mathrm{m}$, isth. 11.5-13.8 $\mu \mathrm{m}$, lob. pol. br. 19.5-23.0 $\mu \mathrm{m}$, th. $27.6 \mu \mathrm{m}$, lg.: br. 1.75-2.16 $\times$.

Occur.: 4rrr, 5rrr, 6rrr, 7rrr, 8rrr, 9rr.

Euastrum bidentatum Näg.

var. speciosum (Boldt) Schmidle (Plate 7, Fig. 10)

Granules on the central protuberance of more or less the same size. Specimen appearance according to Wasylik's (1961a) drawing.

Some authors such as: West, West (1905), Kossinskaya (1960), Förster (1970) consider this taxon as a synonym of the typical variety, whereas the others (Krieger 1937b, Wasylik 1961a, Dubois-Tylski 1969) distinguish it.

Lg. 48.3-50.6 $\mu \mathrm{m}$, br. $32.2 \mu \mathrm{m}$, isth. 6.9-9.2 $\mu \mathrm{m}$, lob. pol. br. 23.0$26.4 \mu \mathrm{m}$, lg.: br. $1.50-1.57 \times$.

Occur.: 2rrr, 7rrr, 8rrr, 9rrr.

Euastrum binale (Turp.) Ehrenb. ex Ralfs

var. binale f. binale (Plate 7, Fig. 13-14)

Lg. 18.4-23.0 $\mu \mathrm{m}$, br. 13.8-18.4 $\mu \mathrm{m}$, isth. 3.4-4.6 $\mu \mathrm{m}$, lob. pol. br. 9.2-11.5 $\mu \mathrm{m}$, lg.: br. 1.25-1.42 $\times$.

Occur.: 1rrrr, 3rrr, 4r, 5rrr, 8rrrr, 9rrr.

f. gutwinskii Schmidle (Plate 7, Fig. 15-22)

The specimens found express high morphological variation. Some have only biundulate basal angles of semicells while the third lower undu-

\section{Plate 6}

Fig. 1. Closterium juncidum var. juncidum. Fig. 2. Cl. directum var. directum. Fig. 3. Cl. macilentum var. macilentum. Fig. 4. Ol. archerianum var. archerianum. Fig. 5. Pleurotaenium ehrenbergii var. ehrenbergii. Fig. 6-7. Pl. rectum var. rectum. Fig. 8. Tetmemorus brebissonii var. minor. Fig. 9. T. brebissonii var. brebissonii. Fig. 10. T. laevis var. laevis. Fig. 11. T. laevis var. intermedius. Fig. 12. T. laevis var. minutus. Fig. 13. T. granulatus var. attenuatus, morpha 1. Fig. 14. T. granulatus var. attenuatus, morpha 2. Fig. 15-17. T. granulatus var. granulatus 
lation is lacking (Plate 7, Fig. 17). In others all undulations are slightly marked (Plate 7, Fig. 18). Similar individuals are described by Růžička (1954).

In Biały tug A bog I found numerous specimens with triundulate basal angles of one semicell (it corresponds to f. gutwinskii), and entire basal angles of another semicell (it corresponds with typical taxon f. binale, Plate 7, Fig. 19). Also intermediate forms with biundulate basal angles of one semicell (Plate 7, Fig. 20) were encountered. Therefore, distinguishment of the typical taxon is disputable. It seems to constitute only a developmental stage of. f. gutwinskii. Then taxon Eu. binale var. binale f. gutwinskii should be raised in rank to a species and, at the same time it should represent the typical taxon.

Sometimes I observed cells in the course of their division (Plate 7, Fig. 21), as well as those whose division was slightly interrupted (Plate 7, Fig. 22). Abnormal division in this taxon is noted fairly often (Kossinskaya 1960, Förster 1970, Palamar-Mordvintseva 1975a and others).

Lg. 24.1-27.6 $\mu \mathrm{m}$, br. 18.4-20.7 $\mu \mathrm{m}$, isth. 4.6-5.7 $\mu \mathrm{m}$, lob. pol. br. 11.5-13.8 $\mu \mathrm{m}$, th. $12.6 \mu \mathrm{m}$, lg.: br. 1.22-1.43 $\times$.

Occur.: 1r, 2rr, 3rr, 4ce, 5rrr, 6c, 7rrr, 8rr, 9rrr.

Euastrum denticulatum (Kirchn.) Gay

var. denticulatum (Plate 7, Fig. 11)

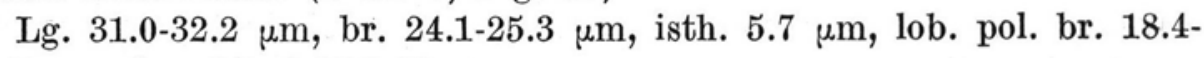
$20.7 \mu \mathrm{m}, \mathrm{lg} .:$ br. $1.27-1.29 \times$.

Occur.: 4rrr, 9rrr.

Euastrum elegans (Bréb.) Kütz. ex Ralfs

var. elegans (Plate 7, Fig. 23)

Lg. 25.3-42.5 $\mu \mathrm{m}$, br. 18.4-27.6 $\mu \mathrm{m}$, isth. 3.4-6.9 $\mu \mathrm{m}$, lob. pol. br. 13.8-18.4 $\mu \mathrm{m}$, lg.: br. 1.38-1.56 $\times$.

Occur.: 4rr, 7rrr, 9rrr.

Euastrum gayanum De Toni

var. gayanum (Plate 7, Fig. 7)

Lg. 11.5-16.1 $\mu \mathrm{m}$, br. 10.3-12.6 $\mu \mathrm{m}$, isth. 2.3-4.6 $\mu \mathrm{m}$, lob. pol. br.

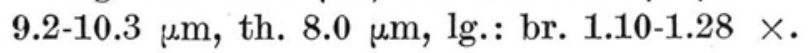

Occur.: 7rrr, 8rrr, 9rrr.

\section{Plate 7}

Fig. 1. Euastrum affine var. affine. Fig. 2. E. ampullaceum var. ampullaceum. Fig. 3. $E$. ansatum var. ansatum. Fig. 4. E. insulare var. insulare. Fig. 5-6. E. insulare var. silesiacum. Fig. 7. E. gayanum var. gayanum. Fig. 8. E. sublobatum var. sublobatum. Fig. 9. E. oblongum var oblongum. Fig. 10. E. bidentatum var. speciosum. Fig. 11. E. denticulatum var. denticulatum. Fig. 12. E. insigne var. insigne. Fig. 13-14. E. binale var. binale f. binale. Fig. 15-22. E. binale var. binale f. gutwinskii. Fig. 23. E. elegans var. elegans. Fig. 24. Micrasterias crux-melitensis var. crux-melitensis 
PLATE 7

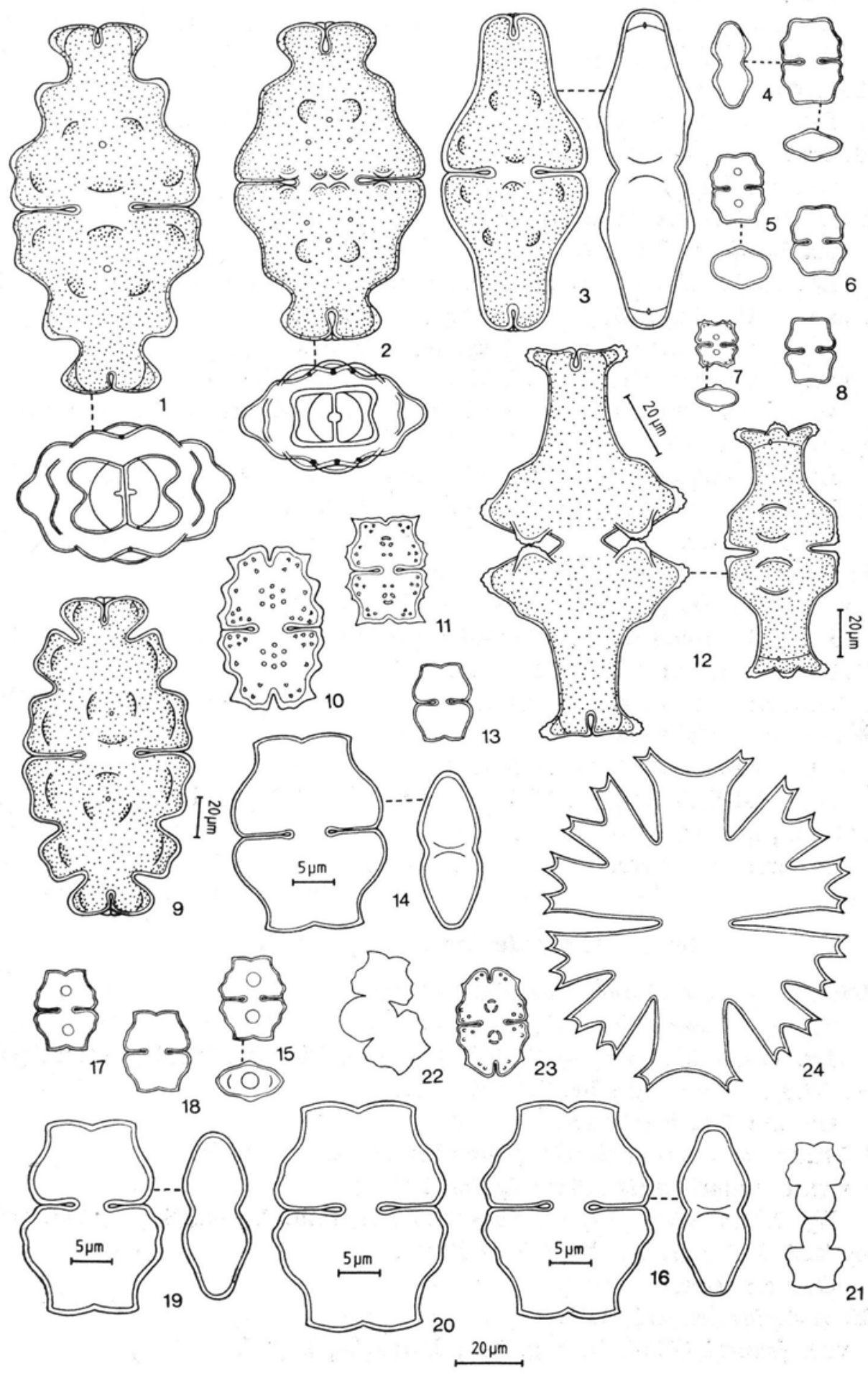


Euastrum insigne Hass. ex Ralfs

var. insigne (Plate 7, Fig. 12)

Lateral lobes in midregion with distinct depression. The specimens fit those found by Ryppowa (1927) and Grönblad (1921), who treats them as a form.

Lg. 102.3-133.4 $\mu \mathrm{m}$, br. 50.6-64.4 $\mu \mathrm{m}$, isth. 13.8-20.7 $\mu \mathrm{m}$, lob. pol. br. 27.6-35.6 $\mu \mathrm{m}$, lg.: br. 1.79-2.15 $\times$.

Occur.: 3rrr.

Euastrum insulare (Wittr.) Roy

var. insulare (Plate 7, Fig. 4)

Lg. 23.0-25.3 $\mu \mathrm{m}$, br. 16.1-18.4 $\mu \mathrm{m}$, isth. $4.6 \mu \mathrm{m}$, lob. pol. br. 11.5$13.8 \mu \mathrm{m}$, th. $9.2-10.3 \mu \mathrm{m}$, lg.: br. 1.34-1.50 $\times$.

Occur.: 1rr, 2rrr, 3r, 4ce, 5rrr, 6c, 8rrr, 9rrr.

var. silesiacum (Grönbl.) W. Krieg. (Plate 7, Fig. 5-6)

In some specimens lateral lobes of each semicell are differently formed. (Plate 7, Fig. 6)

Lg. 20.7-23.0 $\mu \mathrm{m}$, br. 14.9-18.4 $\mu \mathrm{m}$, isth. 3.4-4.6 $\mu \mathrm{m}$, lob. pol. br. 10.3-13.8 $\mu \mathrm{m}$, th. $12.6 \mu \mathrm{m}$, lg.: br. $1.20-1.29 \times$.

Occur.: 1rrr.

Euastrum oblongum (Grev.) Ralfs ex Ralfs

var. oblongum (Plate 7, Fig. 9)

Lg. 144.9-159.8 $\mu \mathrm{m}$, br. 75.9-86.2 $\mu \mathrm{m}$, isth. 20.7-26.4 $\mu \mathrm{m}$, lob. pol. br. 41.4-46.0 $\mu \mathrm{m}$, lg.: br. 1.80-1.97 $\times$.

Occur.: 3rrr, 7rrr, 8rrr, 9rrr.

Euastrum sublobatum Bréb.

var. sublobatum (Plate 7, Fig. 8)

Lg. 18.4-19.5 $\mu \mathrm{m}$, br. 13.8-16.1 $\mu \mathrm{m}$, isth. $4.6 \mu \mathrm{m}$, lob. pol. br. 11.5 $12.6 \mu \mathrm{m}$, lg.: br. 1.21-1.33 $\times$.

Occur.: 8rrr, 9rrr.

\section{Genus: Micrasterias Agardh ex Ralfs 1848}

Miorasterias americana Ehrenb. ex Ralfs

var. americana (Plate 11, Fig. 17)

Lg. 126.5-159.8 $\mu \mathrm{m}$, br. 112.7-132.2 $\mu \mathrm{m}$, isth. 23.0-27.6 $\mu \mathrm{m}$, lob. pol. br. 59.8-73.6 $\mu \mathrm{m}, \mathrm{lg}$.: br. 1.10-1.25 $\times$.

Occur.: 7rr, 8rrr, 9rrr.

Micrasterias crux-melitensis (Ehrenb.) Hass. ex Ralfs

var. crux-melitensis (Plate 7, Fig. 24)

Lg. 102.3-105.8 $\mu \mathrm{m}$, br. 90.8-96.6 $\mu \mathrm{m}$, isth. 13.8-21.8 $\mu \mathrm{m}$, lob. pol. br. 34.5-39.1 $\mu \mathrm{m}$, lg.: br. 1.10-1.12 $\times$.

Occur.: 7rrr.

Micrasterias jenneri Ralfs

var. jenneri (Plate 9, Fig. 1-5; Plate 10, Fig. 1) 
Shape of semicells in vertical view fits Grönblad's (1924; Plate 2, Fig. 21) drawing. It is similar to Ralfs's (1848) drawing (Plate 11, Fig. 1d), on the other hand, it differs from the data of West, West (1905; Plate 43, Fig. 2)

From the observations on relatively numerous population of $M$. jenneri it may be inferred that the distinguished taxon - var. simplex $\mathrm{W}$. West (Plate 9, Fig. 1) is a developmental stage of the cell of var. jenneri, derived from generative reproduction. Therefore, it should be treated as a synonym of the typical variety (Plate 10, Fig. 1). Thorough motivation of this statement may be found in Tomaszewiez's (1977) work. In this respect the diagnosis of $M$. jenneri Ralfs var. jenneri should be completed with a note that lateral lobes at early developmental stages may be divided to the second or, third order, whereas cell wall may be smooth or punctate.

Lobi laterales cellularum in stadiis juvenalibus oum 2 vel 4 lobuletis sunt. Membrana cellularis glabra vel punctata est.

In some specimens apices of polar lobes have got not only median retuse, but also two distinct lateral ones (Plate 9, Fig. 5). Similar specimens have been reported by Krieger (1933a).

Lg. 135.7-172.5 $\mu \mathrm{m}$, br. 92.0-118.4 $\mu \mathrm{m}$, isth. 16.1-25.3 $\mu \mathrm{m}$, lob. pol. br. $55.2-74.8 \mu \mathrm{m}$, th. $51.7 \mu \mathrm{m}$, lg.: br. 1.33-1.50 $\times$.

Occur.: 1rir, 2rrr, 3rir.

Micrasterias papillifera Bréb.

var. papillifera (Plate 8, Fig. 4)

Lg. 121.9-125.4 $\mu \mathrm{m}$, br. 115.0-116.1 $\mu \mathrm{m}$, isth. 18.4-20.7 $\mu \mathrm{m}$, lob. pol. br. $36.8-46.0 \mu \mathrm{m}$, lg.: br. 1.06-1.08 $\times$.

Occur.: 7rrr, 8rrr, 9rrr.

var. glabra Nordst. (Plate 8, Fig. 5)

Lg. $123.0 \mu \mathrm{m}$, br. $119.6 \mu \mathrm{m}$, isth. $19.5 \mu \mathrm{m}$, lob. pol. br. $36.8 \mu \mathrm{m}$, lg.: br. $1.03 \times$.

Occur.: 8rir, 9rrr.

Micrasterias rotata (Grev.) Ralfs ex Ralfs

var. rotata f. rotata (Plate 8, Fig. 1)

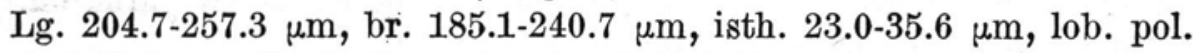
br. $44.8-59.8 \mu \mathrm{m}$, th. $51.7 \mu \mathrm{m}$, lg.: br. 1.00-1.26 $\times$.

Occur.: 4rrr, 5rrr, 6rrr, 7rrr, 8rrr, 9rrr.

f. evoluta Turn. (Plate 8, Fig. 3)

Lg. 244.8-265.6 $\mu \mathrm{m}$, br. 224.1-244.8 $\mu \mathrm{m}$, isth. 27.6-36.8 $\mu \mathrm{m}$, lob. pol. br. 57.5-70.1 $\mu \mathrm{m}$, lg.: br. 1.08-1.15 $\times$.

Occur.: 4rrr, 7rrr, 8rrr, 9rrr.

f. granulata W. West (Plate 8, Fig. 2)

A cell smaller with slightly wider polar lobes than those given by Roll (1924, after Kossinskaya 1960). 
Lg. $278.0 \mu \mathrm{m}$, br. $249.0 \mu \mathrm{m}$, isth. $26.4 \mu \mathrm{m}$, lob. pol. br. $72.4 \mu \mathrm{m}$, lg.: br. $1.12 \times$.

\section{Occur.: 9rri.}

Micrasterias thomasiana Arch.

var. thomasiana (Plate 8, Fig. 6)

Lg. 197.8-236.5 $\mu \mathrm{m}$, br. 177.1-209.3 $\mu \mathrm{m}$, isth. 20.7-26.4 $\mu \mathrm{m}$, lob. pol. br. 43.7-52.9 $\mu \mathrm{m}$, lg.: br. 1.09-1.14 $\times$.

Occur.: 1rrr, 5rrr, 6rr, 7rrr, 8rrr, 9rrr.

var. notata (Nordst.) Grönbl. (Plate 8, Fig. 7-8)

Individuals morphologicly varied. Two groups may be distinguished among them:

a) Cells usually fit the description of var. notata. Incisions between polar and lateral lobes and between lateral lobes of the second and third order are closed and only slightly open in the ends. At the semicells base three distinct papillae are visible. Number of the end lobules -16 . Such specimens are described by: Krieger (1939), Lenzenweger (1965) and Förster (1970), (Plate 8, Fig. 7).

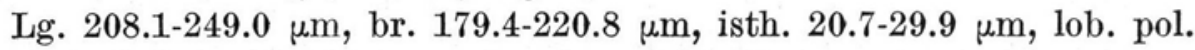
br. $46.0-50.6 \mu \mathrm{m}$, th. $46.0 \mu \mathrm{m}$, lg.: br. 1.10-1.19 $\times$.

Occur.: 5rrr, 6r, 7rrr, 8rrr, 9rrr.

b) morpha (Plate 8 , Fig. 8)

Incisions between polar lobe and lateral lobes halfly open, those between lateral lobes of the second order open in the ends, whereas those between lobes of the third order wholly open. No papillae were observed. Number of the end lobules - 15. Specimens similar to those described by West, West (1905). However, their incisions between polar and lateral lobes are differently formed.

Lg. 218.5-232.4 $\mu \mathrm{m}$, br. 189.7-199.2 $\mu \mathrm{m}$, isth. $27.6 \mu \mathrm{m}$, lob. pol. br. $54.0 \mu \mathrm{m}$, lg.: br. 1.15-1.17 $\times$.

Occur.: 8rrr.

In the studies no numerous populations were found. Only single specimens were usually encountered. Therefore, it is difficult to decide whether two groups distinguished above are two distinct taxonomical units, or difference in their shape lies in the intrapopulational variation.

Micrasterias truncata Corda ex Bréb.

var. truncata (Plate 10, Fig. 2-4)

On the grounds of Tomaszewicz's (1973b) work I have included all found representatives of var. crenata (Bréb.) Grönbl. (Table 10, Fig. 3)

\section{Plate 8}

Fig. 1. Micrasterias rotata var. rotata f. rotata. Fig. 2. M. rotata var. rotata f. granulata. Fig. 3. M. rotata var. rotata f. evoluta. Fig. 4. M. papillifera var. papillifera. Fig. 5. M. papillifera var. glabra. Fig. 6. M. thomasiana var. thomasiana. Fig. 7. M. thomasiana var. notata. Fig. 8. M. thomasiana var. notata, morpha 
PLATE 8
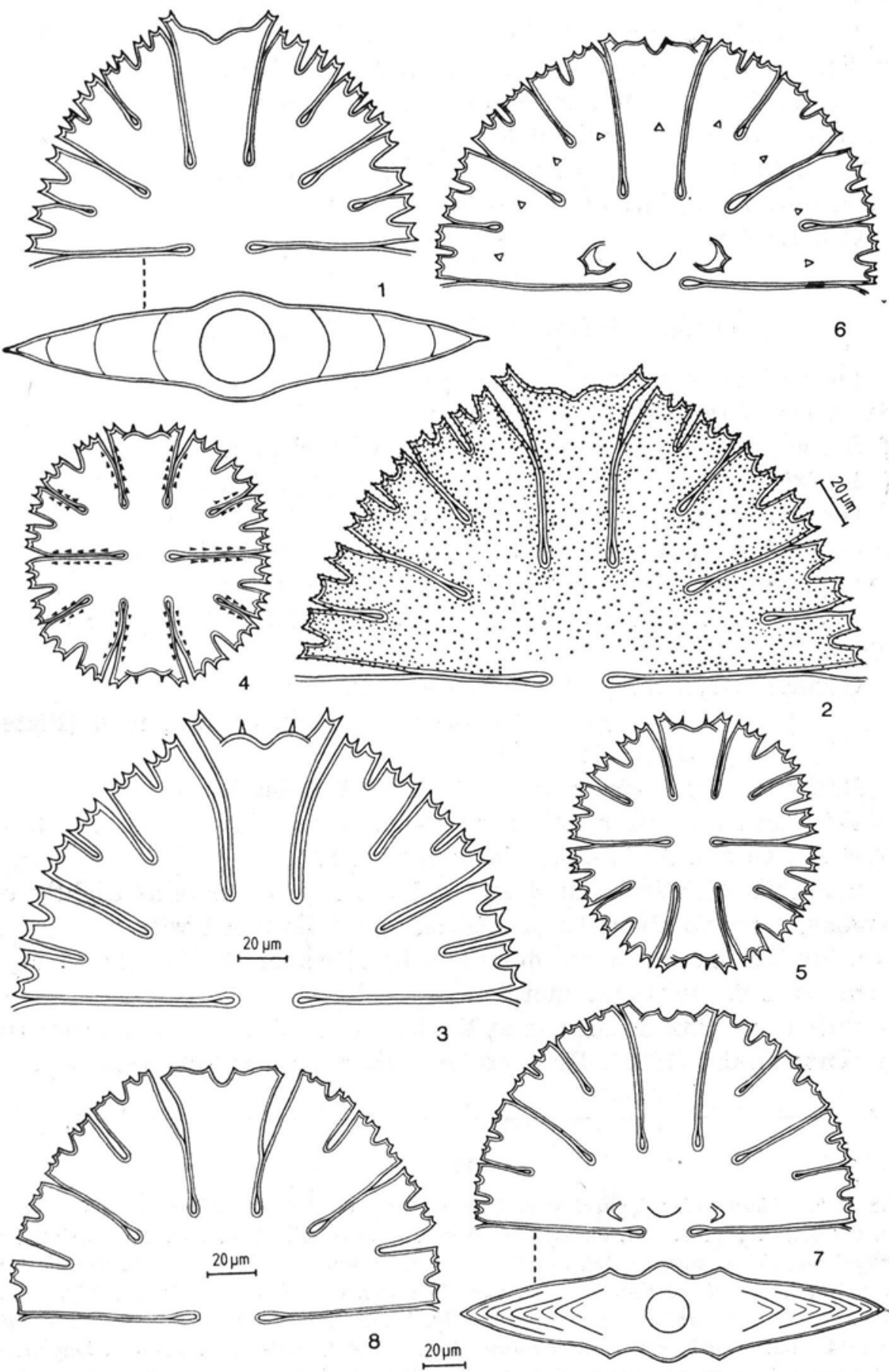

2
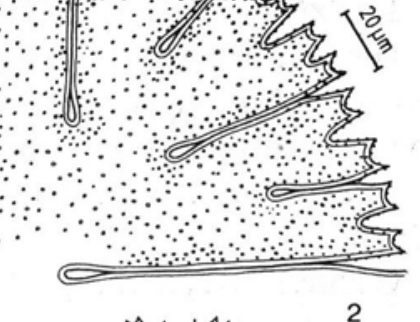
and var. neodamensis (A. Braun) W. Krieg. (Plate 10, Fig. 4,) in the typical variety.

Lg. 87.4-109.2 $\mu \mathrm{m}$, br. 79.3-98.9 $\mu \mathrm{m}$, isth. 13.8-25.3 $\mu \mathrm{m}$, lob. pol. br. 52.9-73.6 $\mu \mathrm{m}$, th. $35.6 \mu \mathrm{m}$, lg.: br. $1.00-1.18 \times$.

Occur.: 1rr, 2rr, 3rr, 4c, 5rr, 6ce, 7rr, 8rr, 9rr.

var. bahusiensis Wittr. (Plate 10, Fig. 5)

Lg. 90.8-119.6 $\mu \mathrm{m}$, br. 72.4-92.0 $\mu \mathrm{m}$, isth. 18.4-23.0 $\mu \mathrm{m}$, lob. pol. br. 57.5-69.0 $\mu \mathrm{m}$, lg.: br. 1.21-1.30 $\times$.

Occur.: 4rr.

\section{Genus: Actinotaenium (Nägeli) Teiling 1954}

Actinotaenium cruciferum (De Bary) Teil.

var. cruciferum (Plate 9, Fig. 9)

Specimens with a shallow median constriction.

Lg. $25.3 \mu \mathrm{m}$, br. $11.5 \mu \mathrm{m}$, isth. $10.3 \mu \mathrm{m}$, lg.: br. $2.20 \times$.

Occur.: 8rrr.

Actinotaenium cucurbita (Bréb. ex Ralfs) Teil. ex Růž. et Pouz

var. cucurbita f. cucurbita (Plate 9, Fig. 10)

Lg. 39.1-43.7 $\mu \mathrm{m}$, br. 19.5-23.0 $\mu \mathrm{m}$, isth. 17.2-20.7 $\mu \mathrm{m}$, lg.: br. 1.85$2.00 \times$.

Occur.: 1rrr, 2rrr, 3c, 4c, 5rrr, 6cc, 8rrr, 9rrr.

f. inflatum (Gutw.) comb. nov. et stat. nov. (Plate 9, Fig. 11)

Basion.: Disphinctium cucurbita (Bréb.) Reinsch var. inflatum Gutwiński (1909), Flora algarum montium Tatrensium, Bull. Acad. Sci. Cracovie, Cl Sci. Math.-Nat., p. 448, pl. $7: 11$.

Semicells slightly inflated at the base. Lateral margins slightly convergent, concave above basal angles. Apices flattened with rounded angles. Similar specimens are described by Förster (1970), who identified them as Act. cucurbita, morpha.

Specimens with a inflation at the base of semicells were first described by Gutwiński (1909). He considered them as a variety (var. inflatum)

\section{Plate 9}

Fig. 1-5. Micrasterias jenneri var. jenneri. Fig. 6. Actinotaenium cucurbitinum var. cucurbitinum. Fig. 7. Act. cucurbitinum var. minutum. Fig. 8. Act. cucurbitinum var. subpolymorphum. Fig. 9. Act. cruciferum var. cruciferum. Fig. 10. Act. cucurbita var. cucurbita f. cucurbita. Fig. 11. Act. cucurbita var. cucurbita f. inflatum. Fig. 12. Act. cucurbita var. cucurbita f. rotundatum. Fig. 13. Act. cucurbita var. cucurbita f. latius. Fig. 14. Act. cucurbita var. attenuatum. Fig. 15. Act. wollei var. wollei f. longior. Fig. 16. Cosmarium quadratulum. Fig. 17. Co. impressulum var. cosandeyi. Fig. 18. Co. tinctum var. tinctum. Fig. 19. Co. globosum var. globosum f. minor. Fig. 20. Co. exiguum 
PLATE 9

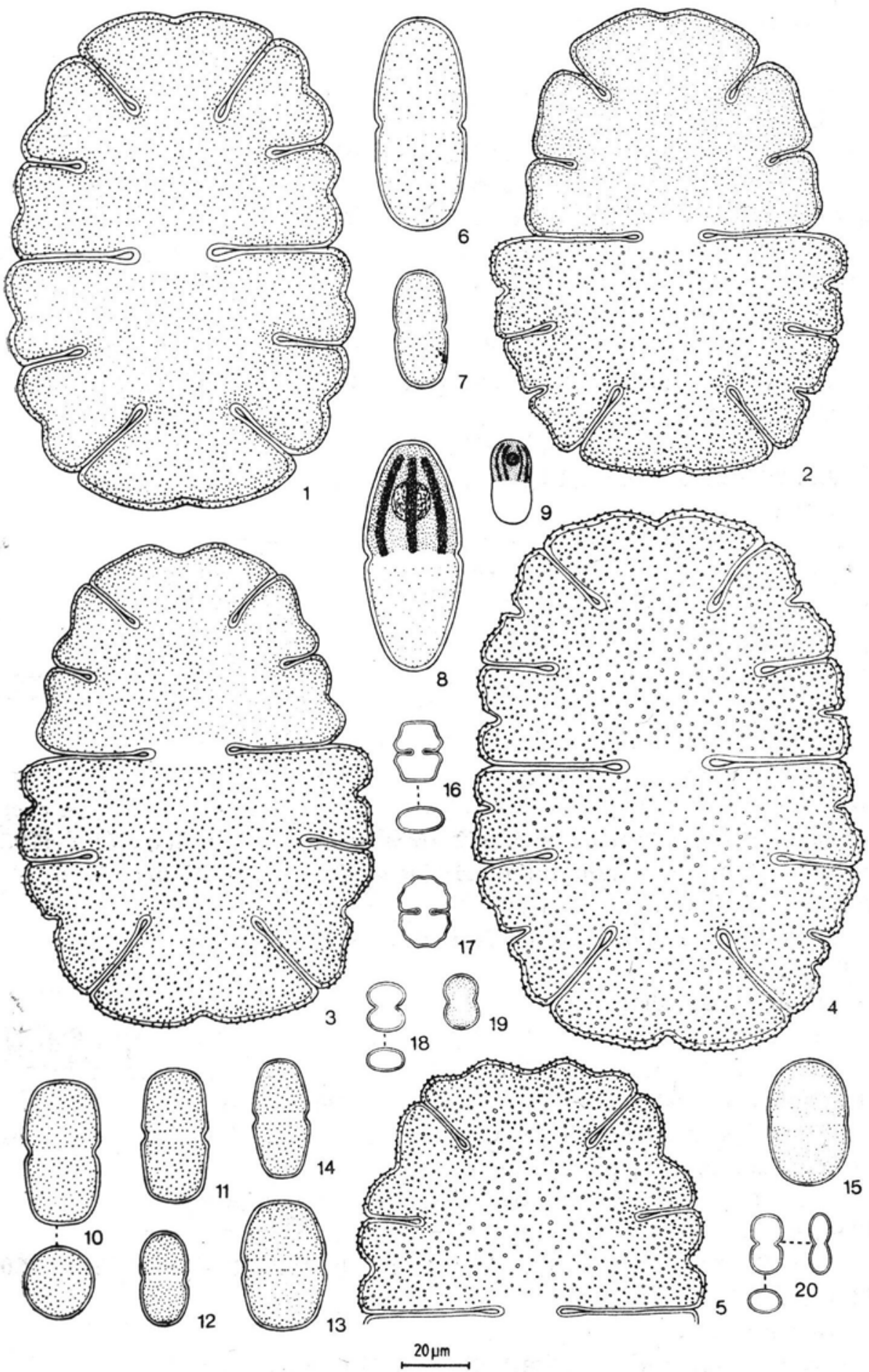


of Disphinctium cucurbita (Bréb.) Reinsch. On the other hand, Teiling (1954) did not classify them as a separate taxon, but only as a synonym of var. attenuatum (G. S. West) Teil.

In the encountered specimens shapes of semicells are more close to that of typical variety and not to var. attenuatum. As it has been already mentioned, lateral margins are only slightly convergent, which also corresponds to Gutwiński's (1909) and Förster's (1970) drawings.

I think that specimens described above should be classified as a separate taxon, but of the rank of form and not variety.

Lg. $34.5-43.7 \mu \mathrm{m}$, br. $19.5-23.0 \mu \mathrm{m}$, isth. 17.2-18.4 $\mu \mathrm{m}$, lg.: br. $1.67-$ $2.00 \times$.

Occur.: 2rrr, 3rrr, 4rrr, 6rrr.

f. latius (W. et G. S. West) Teil. (Plate 9, Fig. 13)

Lg. $39.1 \mu \mathrm{m}$, br. $24.1-25.3 \mu \mathrm{m}$, isth. 23.0-24.1 $\mu \mathrm{m}$, lg.: br. 1.55-1.62 ×. Occur.: 4rrr.

f. rotundatum (W. Krieg.) Teil. (Plate 9, Fig. 12)

Lg. 26.4-27.6 $\mu \mathrm{m}$, br. $13.8 \mu \mathrm{m}$, isth. 11.5-12.6 $\mu \mathrm{m}$, lg.: br. 1.91-2.00 $\times$. Occur.: 3rrrr.

var. attenuatum (G. S. West) Teil. (Plate 9, Fig. 14)

Lg. 33.3-35.6 $\mu \mathrm{m}$, br. 16.1-19.5 $\mu \mathrm{m}$, isth. 13.8-18.4 $\mu \mathrm{m}$, lg.: br. 1.71$-2.14 \times$.

Occur.: 3rrr.

Actinotaenium cucurbitinum (Biss.) Teil.

var. cucurbitinum (Plate 9, Fig. 6)

Specimens of medium size. They correspond to the upper dimension limit of Penium cucurbitinum Biss. f. minor W. et G. S. West. Krieger, Gerloff (1969) treat the above taxon as a synonym of Cosmarium cucurbitinum (Biss.) Lütkem. var. minutum Presc., while Rủžička (1981) includes it in the synonyms of Actinotaenium cucurbitinum (Biss.) Teil. var. cucurbitinum.

Lg. $55.2-62.1 \mu \mathrm{m}$, br. 25.3-26.4 $\mu \mathrm{m}$, isth. 20.7-24.1 $\mu \mathrm{m}$, lg.: br. 2.18$2.35 \times$.

Occur.: 1rrr, 3rrr.

var. minutum (Presc.) Teil. comb. nov. et stat. nov (Plate 9, Fig. 7)

Basion.: Penium cucurbitinum Biss. f. minutum Prescott in Prescott et Magnotta (1935), Notes on Michigan Desmids with descriptions of some species and varieties new to science, Pap. Mich. Acad. Sci. Arts Letters, 20, p. 158, pl. $25: 9$.

Actinotaenium cucurbitinum (Biss.) Teil. var. minutum (Presc.) Teiling 1954, p. 400; combination unvalidly published, Art. 33 Int. Code.

Lg. 33.3-34.5 $\mu \mathrm{m}$, br. 14.9-16.1 $\mu \mathrm{m}$, isth. 12.6-13.8 $\mu \mathrm{m}$, lg.: br. 2.07$2.23 \times$.

Occur.: 4rrr.

var. subpolymorphum (Nordst.) Teil. (Plate 9, Fig. 8) 
Semicells with markedly convergent lateral margins, rounded apices and rarely porous cell wall. Similar specimens described by: Krieger (1933a), Teiling (1954), Krieger, Gerloff (1969) and Förster (1972).

Lg. 66.7-67.8 $\mu \mathrm{m}$, br. 27.6-28.7 $\mu \mathrm{m}$, isth. 25.3-26.4 $\mu \mathrm{m}$, lg.: br. 2.36 $2.42 \times$.

Occur.: 4rrr.

Actinotaenium wollei (W. et G. S. West) Teil. ex Růž. et Pouz.

var. wollei f. longior (Grönbl.) comb. nov. (Plate 9, Fig. 15)

Basion.: Cosmarium wollei (W. et G. S. West) Grönbl. f. longior Grönblad (1947), Desmidiaceen aus Salmi, Acta Soc. pro Fauna et Flora Fenn., 66,1 , p. 25 , pl. $1: 8-9$.

Krieger, Gerloff (1969) quote inadequate Grönblad's work (1947), in which a holotype of this taxon is supposed to be given.

Lg. $36.8 \mu \mathrm{m}$, br. $24.1-25.3 \mu \mathrm{m}$, isth. 23.0-24.1 $\mu \mathrm{m}$, lg.: br. 1.45-1.53 $\times$. Occur.: 3rrr.

\section{Genus: Cosmarium Corda ex Ralfs 1848}

Cosmarium amoenum Bréb.

var. amoenum (Plate 11, Fig. 10-11)

a) Individuals with regularly granulate cell wall, according to the descriptions in the relevant literature (Plate 11, Fig. 10)

Lg. $42.5-57.5 \mu \mathrm{m}$, br. 20.7-32.2 $\mu \mathrm{m}$, isth. 13.8-20.7 $\mu \mathrm{m}$, th. $18.4 \mu \mathrm{m}$, lg.: br. 1.79-2.11 $\times$.

Occur.: 1rrr, 2rrr, 4r, 5rrr, 7rrr, 8rrr, 9rr.

b) morpha (Plate 11, Fig. 11)

Cell wall in the poles with unregular granules. Across a base of semicells a series of longitudinal granules. Specimens similar to Co. pseudamoenum Wille var. basilare Nordst. They differ in a number of granule series across a semicell base and in their shape.

Lg. 41.4-44.8 $\mu \mathrm{m}$, br. $24.1-25.3 \mu \mathrm{m}$, isth. 14.9-17.2 $\mu \mathrm{m}$, lg.: br. 1.72$1.77 \times$.

Occur.: 5rrr.

Cosmarium angulosum Bréb.

var. angulosum (Plate 10, Fig. 18)

Lg. 19.5-21.8 $\mu \mathrm{m}$, br. 12.6-13.8 $\mu \mathrm{m}$, isth. 3.4-5.7 $\mu \mathrm{m}$, lg.: br. 1.50$1.58 \times$.

Occur.: 7rrr, 8rrr, 9rrr.

Cosmarium asphaerosporum Nordst.

var. strigosum Nordst. (Plate 10, Fig. 8)

Lg. $11.5 \mu \mathrm{m}$, br. $10.3 \mu \mathrm{m}$, isth. $4.6 \mu \mathrm{m}$, th. $5.7 \mu \mathrm{m}$, lg.: br. $1.12 \times$.

Occur.: 1rrr.

Cosmarium bioculatum Bréb.

var. bioculatum f. bioculatum (Plate 10, Fig. 11) 
Lg. 14.9-18.4 $\mu \mathrm{m}$, br. 13.8-17.2 $\mu \mathrm{m}$, isth. $4.6-6.9 \mu \mathrm{m}$, th. $6.9 \mu \mathrm{m}$, lg.: br. $1.00-1.08 \times$.

Occur.: 2rrr, 4r, 7rr, 8rrr, 9rrr.

f. depressum Schaarschm. (Plate 10, Fig. 10)

Lg. 12.6-13.8 $\mu \mathrm{m}$, br. 13.8-14.9 $\mu \mathrm{m}$, isth. 3.4-4.6 $\mu \mathrm{m}$, th. $5.7 \mu \mathrm{m}$, lg.: br. $0.91-1.00 \times$.

\section{Occur.: 9rr.}

Cosmarium blyttii Wille

var. novae-sylvae W. et G. S. West (Plate 11, Fig. 8)

Cells with two series of granules within each marginal crenation. Drawings similar to those of Hirano (1972) and R ůžička (1973a).

Lg. 20.7-21.8 $\mu \mathrm{m}$, br. 17.2-19.5 $\mu \mathrm{m}$, isth. 4.6-5.7 $\mu \mathrm{m}$, lg.: br. 1.12-1.20 $\times$.

Occur.: 7rrr, 9rr.

Cosmarium contractum Kirchn.

var. contractum (Plate 11, Fig. 1)

Semicells in vertical view more widely elliptic than those of West, West (1905) and rather similar to those of Grönblad (1960; Plate 3, Fig. 48).

Lg. 39.1-42.5 $\mu \mathrm{m}$, br. 25.3-27.6 $\mu \mathrm{m}$, isth. 6.9-9.2 $\mu \mathrm{m}$, th. $23.0 \mu \mathrm{m}$, lg.: br. $1.42-1.55 \times$.

Occur.: 4r.

var. minutum (Delp.) W. et G. S. West (Plate 11, Fig. 2)

Semicells in vertical view narrowly elliptic.

Lg. 23.0-25.3 $\mu \mathrm{m}$, br. 18.4-20.7 $\mu \mathrm{m}$, isth. 4.6-6.9 $\mu \mathrm{m}$, th. $12.6 \mu \mathrm{m}$, lg.: br. $1.22-1.25 \times$.

Occur.: 7rrr, 9rrr.

Cosmarium elegantissimum Lund.

var. simplicius W. et G. S. West (Plate 11, Fig. 12)

Cell wall sculpture fits Růžička's (1973a; Plate 10, Fig. 5) drawing. Förster (1970), on the grounds of Grönblad's (1931) work, treats this taxon as a unit of the species rank - Cosmarium simplicius (W. et G. S. West) Grönbl.

Lg. 43.7-48.3 $\mu \mathrm{m}$, br. 20.7-23.0 $\mu \mathrm{m}$, isth $18.4-20.7 \mu \mathrm{m}$, lg.: br. 2.04$2.11 \times$.

Occur.: 7rrr, 9rrr.

Cosmarium exiguum Arch.

var. exiguum (Plate 9, Fig. 20)

Semicell apices markedly convex. In face view the specimens fit the drawing of Co. exiguum, form in Grönbland's et al. (1968; Plate 6, Fig. 87) work. They differ in somewhat smaller dimensions and narrower semicells in side view.

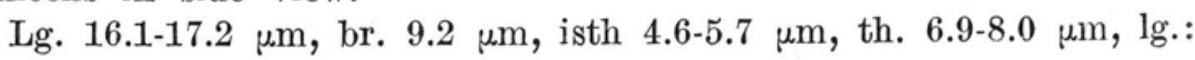
br. $1.75-1.87 \times$.

Occur.: 9rrr. 
Cosmarium formosulum Hoff

var. formosulum (Plate 11, Fig. 9)

Lg. 40.2-43.7 $\mu \mathrm{m}$, br. $36.8 \mu \mathrm{m}$, isth. 9.2-11.5 $\mu \mathrm{m}$, ap. 14.9-16.1 $\mu \mathrm{m}$, lg.: br. 1.09-1.19 $\times$.

Occur.: 5rrr.

Cosmarium globosum Bulnh.

var. globosum f. minor Boldt (Plate 9, Fig. 19)

Lg. 16.1-20.7 $\mu \mathrm{m}$, br. 10.3-12.6 $\mu \mathrm{m}$, isth. 8,0-10.3 $\mu \mathrm{m}$, lg.: br. 1.56$1.64 \times$.

Occur.: 9rrr.

Cosmarium impressulum Elfv.

var. cosandeyi Růž. (Plate 9, Fig. 17)

Cells smaller than in the typical variety, they fit those of var. cosandeyi in R užička's $(1972,1973 a)$ works.

Specimens with dimensions below lower limit of those for var. impressulum (West, West 1908) are described by many authors (Taylor 1934, Hirano 1957a, Palamar 1960, Islam 1970, Mix 1970).

I think if big (that correspond to var. impressulum) and small (that correspond to var. cosandeyi) individuals are found in the same habitat, they occupy the same ecological niche and the difference between them lies (according to $R$ ůžička 1972) only in size ( $\mathrm{I}$ do not have neither original diagnosis, nor holotype - var. minor Cosandey (1934), then var. cosandeyi ( = var. minor Cosandey 1934) ought to be treated as a synonym of var. impressulum.

In the area studied I observed only small individuals, and scarce.

Lg. $20.7 \mu \mathrm{m}$, br. 14.9-16.1 $\mu \mathrm{m}$, isth. 3.4-4.6 $\mu \mathrm{m}$, lg.: br. 1.29-1.39 $\times$.

Occur.: 7rrr.

Cosmarium margaritiferum Menegh. ex Ralfs

var. margaritiferum, morpha (Plate 11, Fig. 13)

Individual size slightly smaller than literature data. Semicells more pyramidate than those in drawings of: West, West (1912), Wasylik (1961a), Prescott (1966), Förster (1970), Růžička (1973a) and Coesel (1979b). On the other hand, they fit descriptions of: Migula (1907), Hirano (1957b) and Lind, Brook (1980).

Lg. 40.2-47.1 $\mu \mathrm{m}$, br. 29.9-35.6 $\mu \mathrm{m}$ isth. 9.2-11.5 $\mu \mathrm{m}$, th. $21.8 \mu \mathrm{m}$, lg.: br. 1.31-1.34 $\times$.

Occur.: 4r.

Cosmarium minimum W. et G. S. West

var. minimum (Plate 10, Fig. 12-13)

a) morpha 1 (Plate 10, Fig. 12)

Cells relatively longer than those described in the literature by: West, West (1908), Hirano (1957a, 1974), and Krieger, Gerloff (1969). Length-breadth ratio fits rather that of var. subrotundatum W. et G. S. West, whereas shape that of var. minimum. 
Lg. 11.5-14.9 $\mu \mathrm{m}$ br. $9.2-12.6 \mu \mathrm{m}$, isth. $3.4 \mu \mathrm{m}$, th. $5.7 \mu \mathrm{m}$, lg.: br. 1.18-1.25 $\times$.

Occur.: 7rrr, 9rrr.

b) morpha 2 (Plate 10, Fig. 13)

Individuals closest to the encountered specimens of Co. minimum var. minimum, morpha 1 . They differ from them in more open, acute-angled sinus and wider isthmus. In their appearance they resemble the drawing of Co. coarctatum W. West in Grönblad's (1942; Plate 2, Fig. 17) work. I consider this drawing to differ from the diagnosis of above taxon given by West, West (1908) and Krieger, Gerloff (1965).

Lg. 9.2-13.8 $\mu \mathrm{m}$, br. 10.3-12.6 $\mu \mathrm{m}$, isth. $5.7 \mu \mathrm{m}$, th. $5.7 \mu \mathrm{m}$, lg.: br. $0.89-1.12 \times$.

Occur.: 4rr, $7 \mathrm{rr}$.

Cosmarium moniliforme Turp. ex Ralfs

var. moniliforme (Plate 10, Fig. 17)

Lg. $32.2 \mu \mathrm{m}$, br. 17.2-18.4 $\mu \mathrm{m}$, isth. $9.2 \mu \mathrm{m}$, th. 17.2-18.4 $\mu \mathrm{m}$, lg.: br. $1.75-1.87 \times$.

Occur.: 4rrr.

var. panduriforme (Heimerl) Schmidle (Plate 10, Fig. 16)

Lg. 18.4-20.7 $\mu \mathrm{m}$, br. 10.3-11.5 $\mu \mathrm{m}$, isth. 6.9-9.2 $\mu \mathrm{m}$, th. 10.3-11.5 $\mu \mathrm{m}$, lg.: br. $1.79-1.80 \times$.

Occur.: 8rrr.

Cosmarium praemorsum Bréb.

var. praemorsum (Plate 11, Fig. 14)

Cell wall sculpture according to drawings of: West, West (1908), Förster (1970) and Lind, Brook (1980).

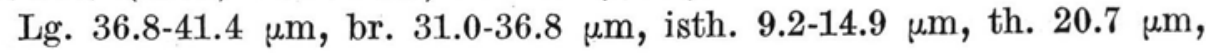
lg.: br. 1.12-1.19 $\times$.

Occur.: 9rrr.

Cosmarium pseudoornatum Eichl. et Gutw.

var. pseudoornatum (Plate 11, Fig. 16)

Lg. 29.9-34.5 $\mu \mathrm{m}$, br. 31.0-32.2 $\mu \mathrm{m}$, isth. 9.2-10.3 $\mu \mathrm{m}$, th. $16.1 \mu \mathrm{m}$, lg.: br. $0.96-1.07 \times$.

Occur.: 5rrr, 7rrr.

\section{Plate 10}

Fig. 1. Micrasterias jenneri var. jenneri. Fig. 2-4. M. truncata var. truncata. Fig. 5. M. truncata var. bahusiensis. Fig. 6. Cosmarium regnellii var. regnellii. Fig. 7. Co. regnellii var. pseudoregnellii. Fig. 8. Co. asphaerosporum var. strigosum. Fig. 9. Co. subarctoum var. subarctoum, morpha. Fig. 10. Co. bioculatum var. bioculatum f. depressum. Fig. 11. Co. bioculatum var. bioculatum f. bioculatum. Fig. 12. Co. minimum var. minimum, morpha 1. Fig. 13. Co. minimum var. minimum, morpha 2. Fig. 14-15. Co. spec. Fig. 16. Co. moniliforme var. panduriforme. Fig. 17. Co. moniliforme var. moniliforme. Fig. 18. Co. angulosum var. angulosum 
PLATE 10

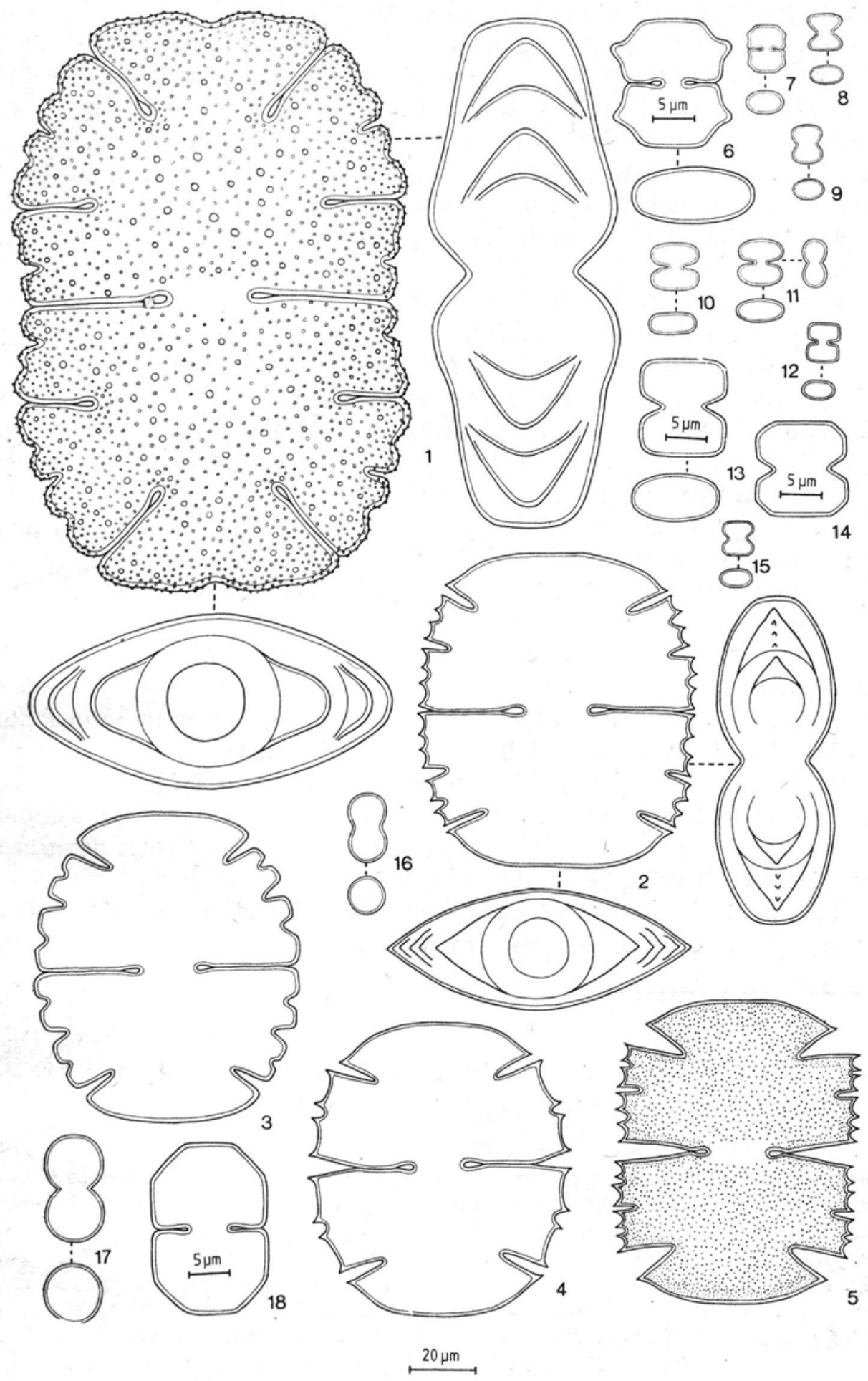


Cosmarium pseudopyramidatum Lund.

var. pseudopyramidatum, morpha (Plate 11, Fig. 5)

Cells relatively longer than typical specimens. Semicells widened toward a base, sides straight, apices and angles rounded. Cell shape fits that of specimens found by Förster (1970) and Oleksowicz (1978). Individual appearance is nearly identical with drawings of Ito (1965a) and Watanabe et al. (1979), the only difference lies in the apex shape truncate not rounded as in my specimens.

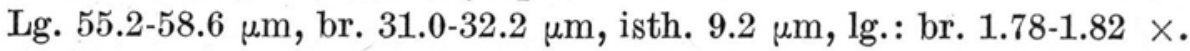

Occur.: 3rrr.

Cosmarium pyramidatum Bréb.

var. pyramidatum (Plate 11, Fig. 6)

Semicell shape in a side view differs from that described by West, West (1905). Semicells are widely not longitudionally elliptic and fit Hirano's (1957a) drawing. Its appearance fits also the drawing of Co. pyramidatum Bréb. var. maximum (Borge) W. Krieg. et Gerloff by Borge published in the work of Krieger and Gerloff (1965).

Lg. 64.4-73.6 $\mu \mathrm{m}$, br. 42.5-47.1 $\mu \mathrm{m}$, isth. 11.5-16.1 $\mu \mathrm{m}$, th. $25.3 \mu \mathrm{m}$, lg.: br. $1.47-1.62 \times$.

Occur.: 1rrr, 2rrr, 3rrr, 4rr, 5rrr, 6rrr, 7rrr, 8rrr, 9rrr. Cosmarium quadratulum (Gay) De Toni (Plate 9, Fig. 16)

West, West (1908) drew various drawings of Co. quadratulum (Plate 72, Fig. 33 and Plate 93, Fig. 4). According to Grönblad (1921) only specimens in Plate 72, Fig. 33 fit Gay's holotype, whereas those in Plate 93, Fig. 4 differ from it especially in the lack of open sinus. The specimens encounterd by me are similar to a drawing from Plate 93, Fig. 4 (West, West 1908). They differ in longer cells, apical angles rather rounded and somewhat convex apices, without retuse. Similar specimens are described by Hirano (1957a, 1974).

Probably, individuals of Co. quadratulum, described by Wests that differ from the Gay's holotype are its variety. It is possible that both

\section{Plate 11}

Fig. 1. Cosmarium contractum var. contractum. Fig. 2. Co. contractum var. minutum. Fig. 3. Co. subtumidum var. subtumidum. Fig. 4. Co. quadratum var. guadratum, morpha. Fig. 5. Co. pseudopyramidatum var. pseudopyramidatum, morpha. Fig. 6. Co. pyramidatum var. pyramidatum. Fig. 7. Co. ralfsii var. ralfsii. Fig. 8. Co. blyttii var. novae-sylvae. Fig. 9. Co. formosulum var. formosulum. Fig. 10. Co. amoenum var. amoenum. Fig. 11. Co. amoenum var. amoenum, morpha. Fig. 12. Co. elegantissimum var. simplicius. Fig. 13. Co. margaritiferum var. margaritiferum, morpha. Fig. 14. Co. praemorsum var. praemorsum. Fig. 15. Co. tetraophthalmum var. tetraophthalmum. Fig. 16. Co. pseudoornatum var. pseudoornatum. Fig. 17. Micrasterias americana var. americana. Fig. 18. Arthrodesmus tenuissimus var. tenuissimus. Fig. 19. ?Art. trispinatus var. trispinatus. Fig. 20. Art. octocornis var. octocornis. Fig. 21. Xanthidium concinnum var. boldtianum. Fig. 22. X. fasciculatum var. basidentatum 

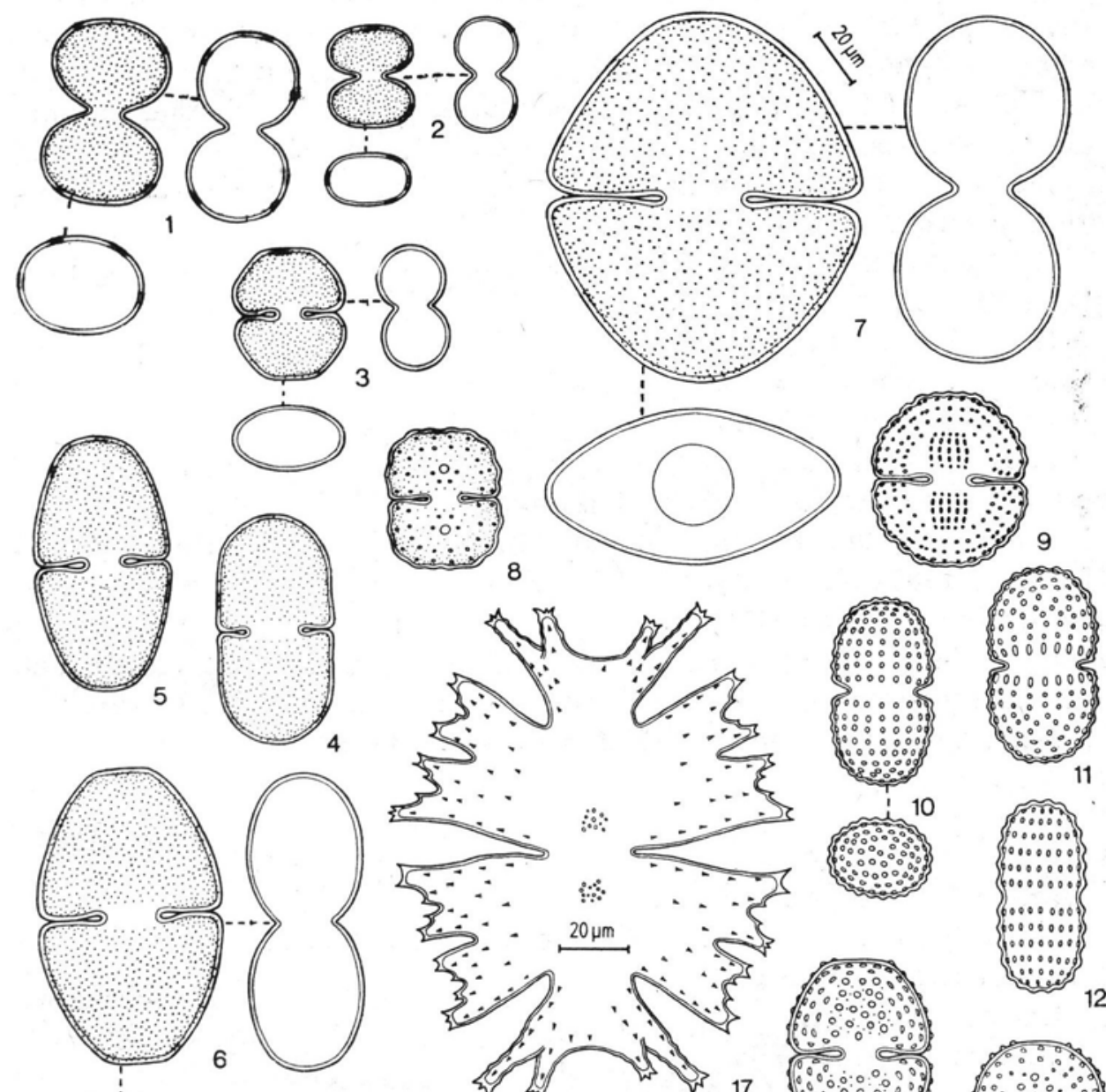

3:- $20 \mathrm{~mm}$

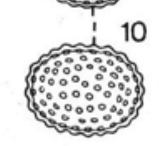

11

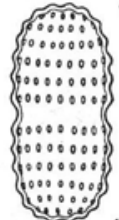

这
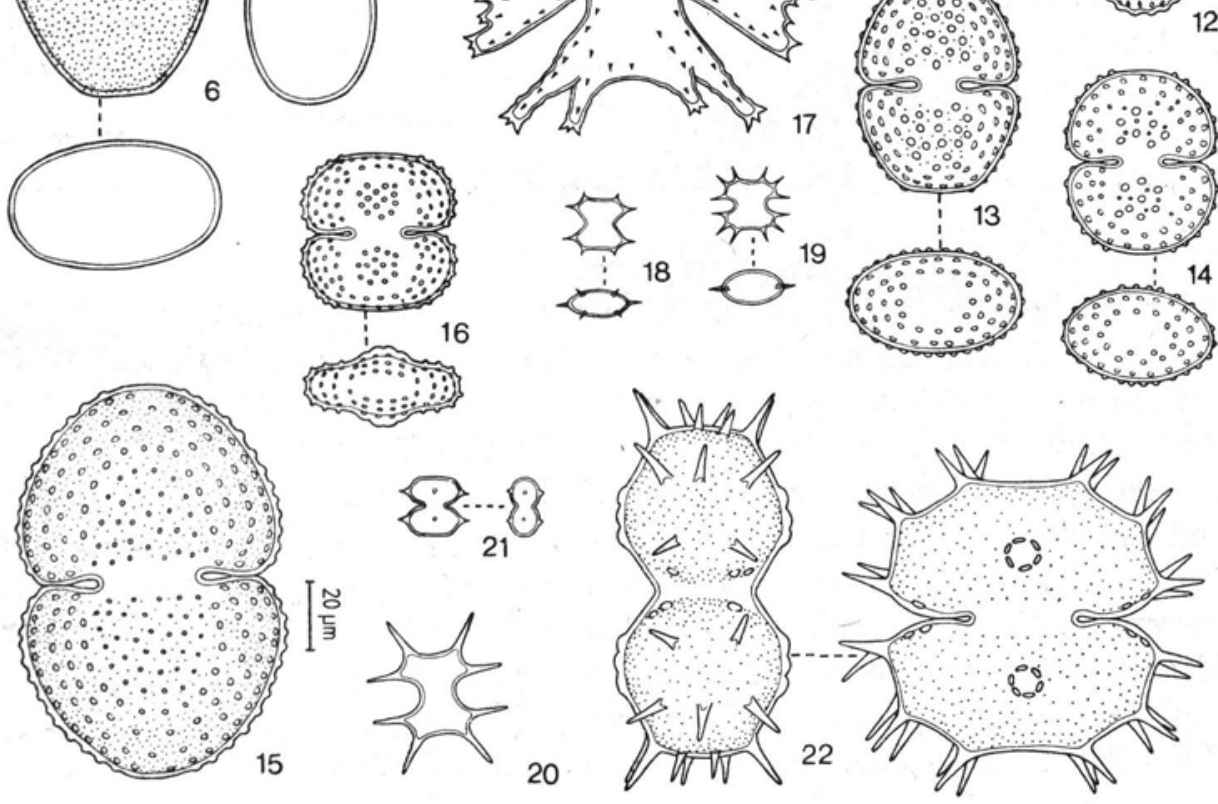
them and those found by me should be classified as var. applanatum Insam et W. Krieg. 1936 (I found its drawings in the works of: Grönblad et al. (1964), and Krieger and Gerloff (1965)). However, as I do not have original diagnosis and drawing, I am not justified, on the grounds of descriptions and drawings mentioned above, to decide whether encountered specimens represent the taxon discussed. I am only sure they cannot be classified as representatives of the typical variety. Therefore, they are classified only to the species rank.

Lg. 16.1-17.2 $\mu \mathrm{m}$, br. $13.8 \mu \mathrm{m}$, isth. 3.4-4.6 $\mu \mathrm{m}$, th. $8.0 \mu \mathrm{m}$, lg.: br. 1.17-1.25 $\times$.

Occur.: 7rrr, 9rrr.

Cosmarium quadratum Ralfs ex Ralfs

var. quadratum, morpha (Plate 11, Fig. 4)

Cells longitudionally elliptic, apex convex, sides straight, but above rounded basal angles slightly depressed.

Semicell outline fits that of individuals found by: Růžička (1956), Hirano (1957a; Plate 24, Fig. 5) and Ito (1968). They are similar to the drawings of Ito (1966a), Růžička (1972: Plate 61, Fig. 5, 1973a; Plate 9, Fig. 9), Agarker, Agarkar (1977), and Hinode (1977). Cell apex of these specimens is less convex than of those found by me.

Lg. 50.6-55.2 $\mu \mathrm{m}$, br. 27.6-31.0 $\mu \mathrm{m}$, isth. 12.6-16.1 $\mu \mathrm{m}$, lg.: br. 1.69$1.92 \times$.

Occur.: 9rrr.

Cosmarium ralfsii Bréb.

var. ralfsii (Plate 11, Fig. 7)

Lg. 110.4-123.0 $\mu \mathrm{m}$, br. 95.4-104.6 $\mu \mathrm{m}$, isth. 19.5-27.6 $\mu \mathrm{m}$, th. 51.7$53.0 \mu \mathrm{m}$, lg.: br. 1.16-1.21 $\times$.

Occur.: 2rrr, 3rrr.

Cosmarium regnellii Wille

var. regnellii (Plate 10, Fig. 6)

Lg. 13.8-16.1 $\mu \mathrm{m}$, br. 12.6-14.9 $\mu \mathrm{m}$, isth. 3.4-4.6 $\mu \mathrm{m}$, th. $6.9 \mu \mathrm{m}$, lg.: br. 1.08-1.10 $\times$.

Occur.: 5rrr, 6rrr, 7rrr, 8rrr, 9rrr.

var. pseudoregnellii (Messik.) W. Krieg. (Plate 10, Fig. 7)

Specimen appearance fits the drawing of Co. braunii (Reinsch) Wolle var. pseudoregnellii Messik. in Laporte's (1931; Plate 11, Fig. 132-134) work. Krieger, Gerloff (1965) classify this drawing as belonging to Co. meneghinii Bréb. var. meneghinii. If compared to the descriptions and drawings of this taxon in works of: West, West (1908), Grönblad (1921), Hirano (1957a) and Messiko mmer (1971a), cells of the specimens encountered and those of Laporte are relatively shorter, the upper part of their sides is wider and shorter, apices are straight. Thus, they differ from the typical specimens of Co. meneghinii. Therefore, after Laporte, I classify them as Co. regnellii (=Co. braunii) var. pseudoregnellii. 
Lg. 13.8-16.1 $\mu \mathrm{m}$, br. 11.5-13.8 $\mu \mathrm{m}$, isth. 3.4-4.6 $\mu \mathrm{m}$, th. $6.9 \mu \mathrm{m}$, lg.: br. $1.17-1.20 \times$.

Occur.: 7rr, 8rrr, 9r.

Cosmarium subarctoum (Lagerh.) Racib.

var. subarctoum, morpha (Plate 10, Fig. 9)

Cell apex flattened, sinus widely open. They are similar to those described by Grönblad (1960), Suxena, Venkateswarlu (1968). Apex in the specimens described by Suxena and Venkateswarlu (1968) had a shallow, hardly visible depression.

Lg. 11.5-12.6 $\mu \mathrm{m}$, br. $9.2-10.3 \mu \mathrm{m}$, isth. $6.9 \mu \mathrm{m}$, lg.: br. 1.22-1.25 $\times$.

Occur.: 5rrr, 7rr.

Cosmarium subtumidum Nordst.

var. subtumidum (Plate 11, Fig. 3)

Lg. 27.6-34.5 $\mu \mathrm{m}$, br. $23.0-29.9 \mu \mathrm{m}$, isth. 6.9-11.5 $\mu \mathrm{m}$, th. $16.1 \mu \mathrm{m}$, lg.: br. $1.15-1.23 \times$.

Occur.: 2rrr, 4r, 6ce, 7rrr, 8rrr, 9rrr.

Cosmarium tetraophthalmum Bréb.

var. tetraophthalmum (Plate 11, Fig. 15)

Lg. 105.8-113.7 $\mu \mathrm{m}$, br. $73.6-78.2 \mu \mathrm{m}$, isth. $23.0-29.9 \mu \mathrm{m}$, lg.: br. 1.44-1.47 $\times$.

Occur.: 1rrr, 7rrr.

Cosmarium tinctum Ralfs

var. tinctum (Plate 9, Fig. 18)

Lg. 13.8-16.1 $\mu \mathrm{m}$, br. $11.5 \mu \mathrm{m}$, isth 6.9-8.0 $\mu \mathrm{m}$, th. $6.9 \mu \mathrm{m}$, lg.: br. 1.20-1.40 $\times$.

Occur.: 2rrr, 7rrr, 8rrr, 9rrr.

Cosmarium sp. (Plate 10, Fig. 14-15)

Semicells rectangular, apical angles markedly truncate, apices straight, sides straight parallel, sinus open, acute-angled, in vertical view semicells elliptic.

Cell shape similar to specimens of Co. minimum W. et G. S. West var. minimum, morpha 2 , encountered by me. They differ only in the truncate apical angles. They are also similar to individuals of $C o$. asphaerosporum Nordst. var. asphaerosporum, but their outline is more straight and cells are different in vertical view.

Lg. 10.3-12.6 $\mu \mathrm{m}$, br. 9.2-11.5 $\mu \mathrm{m}$, isth. $5.7-6.9 \mu \mathrm{m}$, th. $5.7 \mu \mathrm{m}$, lg.: br. $1.10-1.12 \times$.

Occur.: 1rrr, 4rrr.

Genus: Xanthidium Ehrenberg ex Ralfs 1848

Xanthidium antilopaeum Bréb. ex Kütz.

var. antilopaeum (Plate 12, Fig. 1-6)

a) fac. 2 (Plate 12, Fig. 1-2) 
Cell shape in vertical view differs from that of Migula (1907), West, West (1912) and Hirano (1957b). Semicells six-angled, poles straight or faintly concave, they fit descriptions of Grönblad, Rủžička (1959), Bourrelly (1966) and Růžička (1973a, 1973b). Sometimes encountered specimens immersed in gelatinous envelope (Plate 12, Fig. 2).

Lg. 62.1-73.6 $\mu \mathrm{m}$, br. 51.7-62.1 $\mu \mathrm{m}$, isth. 13.8-23.0 $\mu \mathrm{m}$, sp. 10.3-14.9 $\mu \mathrm{m}$, th. 34.5-39.1 $\mu \mathrm{m}$, lg.: br. 1.08-1.25 $\times$.

Occur.: 1rrr, 2rr, 3rrr, 4r, 5rr, 6rrr, 7rrr, 8rrr, 9rrr.

b) fac. 2 morpha (Plate 12, Fig. 3-5)

Cell shape fits that of encountered specimens of var. antilopaeum, fac. 2, but spines at all (Plate 12, Fig. 3-4), or three (Plate 12, Fig. 5) angles are reduced. Cell size oscillates around lower limit of dimensions for var. antilopaeum, fac. 2 . I think all these specimens may be classified as reduced individuals of the typical variety, fac. 2. Moreover, they were found only once and in a small number.

Reduced spines and processes are not rare in desmids. They have been described: for Xanthidium armatum Bréb. ex Bréb. (West, West 1912; Kondo 1975), for Xanthidium subhastiferum W. West (Rosenberg 1944) and for Staurastrum furcatum (Ehrenb. ex Ralfs) Bréb. (Palamar-Mordvintseva 1970).

Lg. 54.0-66.7 $\mu \mathrm{m}$, br. 46.0-52.9 $\mu \mathrm{m}$, isth. 10.3-20.7 $\mu \mathrm{m}$, sp. 2.3-3.4 $\mu \mathrm{m}$, th. $32.2-35.6 \mu \mathrm{m}, \mathrm{lg}$.: br. $1.13-1.30 \times$.

Occur.: 1rrr, 2rrr.

c) fac. 3 (Plate 12, Fig. 6)

Cells smaller than those of West, West (1912), Wasylik (1961b) and Oleksowicz (1978). Even smaller specimens were described by Eichler (1892).

The rank of a taxon is not established yet. Migula (1907) classifies it as a form - f. triquetrum Lund., West, West (1912), Tomaszewicz $(1973 \mathrm{a}, 1974)$ and Oleksowicz (1978) as a variety - var. triquetrum Lund., whereas Růžička (1973b) - as facies 3. These discrepancies arise from the question whether differential character - triangular cells in vertical view - is constant (hereditary) or labile.

Lg. 50.6-57.5 $\mu \mathrm{m}$, br. 46.0-50.6 $\mu \mathrm{m}$, isth. 11.5-19.5 $\mu \mathrm{m}$, sp. 10.3-16.1 $\mu \mathrm{m}$, lg.: br. 1.07-1.22 $\times$.

Occur.: 4rr, 5rrr, 6rrr, 7rrr, 8rrr, 9rrr.

Xanthidium armatum Bréb. ex Bréb.

var. armatum (Plate 12, Fig. 7)

Lg. 105.8-126.5 $\mu \mathrm{m}$, br. 62.1-78.2 $\mu \mathrm{m}$, isth. 28.7-36.8 $\mu \mathrm{m}$, sp. 5.7-11.5 $\mu \mathrm{m}$, th. 52.9-55.2 $\mu \mathrm{m}$, lg.: br. 1.53-1.83 $\times$.

Occur.: 1rrr, 2rrr, 3rr, 4r, 6ce, 7rrr, 8rrr, 9rrr.

Xanthidium concinnum Arch.

var. boldtianum W. West (Plate 11, Fig. 21) 
Lg. $11.5 \mu \mathrm{m}$, br. $11.5-13.8 \mu \mathrm{m}$, isth. $4.6 \mu \mathrm{m}$, sp. $\sim 1 \mu \mathrm{m}$, lg.: br. $0.83-1.00 \times$.

Occur.: $7 \mathrm{r}$.

Xanthidium fasciculatum Ehrenb. ex Ralfs

var. basidentatum (Börges.) Růž. (Plate 11, Fig. 22)

Lg. 55.2-66.7 $\mu \mathrm{m}$, br. 54.0-62.1 $\mu \mathrm{m}$, isth. 16.1-21.8 $\mu \mathrm{m}$, sp. 9.2-10.3 $\mu \mathrm{m}$, lg.: br. 1.02-1.07 $\times$.

Occur.: 7 rrr.

Genus: Arthrodesmus Ehrenberg ex Ralfs 1848

Arthrodesmus octocornis Ehrenb. ex Arch.

var. octocornis (Plate 11, Fig. 20)

Lg. 16.1-23.0 $\mu \mathrm{m}$, br. 12.6-18.4 $\mu \mathrm{m}$, isth. 4.6-6.9 $\mu \mathrm{m}$, sp. 5.7-9.2 $\mu \mathrm{m}$, lg.: br. $1.00-1.43 \times$.

Occur.: 2rrr, 4rr, 7rrr, 8rr, 9rr.

Arthrodesmus tenuissimus Arch.

var. tenuissimus (Plate 11, Fig. 18)

Lg. 9.2-10.3 $\mu \mathrm{m}$, br. 9.2-11.5 $\mu \mathrm{m}$, isth. $5.7 \mu \mathrm{m}$, sp. $2.3 \mu \mathrm{m}$, th. $5.7 \mu \mathrm{m}$, lg.: br. $0.90-1.00 \times$.

Occur.: 8rrr.

? Arthrodesmus trispinatus W. et G. S. West

var. trispinatus (Plate 11, Fig. 19)

Specimens found are symmetrical, spines located regularly. They fit the description and drawing of Arthrodesmus trispinatus of West, West (1912; Plate 117, Fig. 7). The authors mentioned that they did not find any living cell and they could not determine a kind of a chromatophore. Therefore, the above taxon may also belong to genus Tetraëdron.

Teiling (1946) has described asymmetrical individuals with unregularly distributed spines, which fit the drawing of Art. trispinatus by Gutwiński (1909), but with chromatophore of Tetraëdron type. He classifies them to Tetraëdron trispinatum (W. et G. S. West) Hub.-Pestal. At the same time he questions affiliation of West's specimens to genus Arthrodesmus, because the diagnosis was formed on the grounds of empty cells with the incidental symmetry.

On the grounds of two specimens found, moreover without chromatophores it is difficult to affiliate them properly. I classify them in genus Arthrodesmus as their appearance fits the description and drawing of Wests and differs from that of Teiling. Taxa described by Wests and Teiling belong to two different orders. Merely on the grounds of cell shape one cannot decide about its affiliation and about synonymity of both taxa, moreover as some Tetraëdron species have cells of similar shape as Arthrodesmus species. 
Lg. 9.2-11.5 $\mu \mathrm{m}$, br. $11.5 \mu \mathrm{m}$, isth. $6.9 \mu \mathrm{m}$, sp. 2.3-3.4 $\mu \mathrm{m}$, th. $6.9 \mu \mathrm{m}$, lg.: br. $0.80-1.00 \times$.

Occur.: 8rrr.

\section{Genus: Staurodesmus Teiling 1948}

Staurodesmus connatus (Lund.) Thom.

var. connatus (Plate 12, Fig. 14)

Lg. $20.7 \mu \mathrm{m}$, br. 19.5-20.7 $\mu \mathrm{m}$, isth. $6.9 \mu \mathrm{m}$, sp. $6.9 \mu \mathrm{m}$, lg.: br. 1.00$1.06 \times$.

Occur.: 7rrr, 9rrr.

Staurodesmus dejectus (Bréb. ex Bréb.) Teil.

var. dejectus (Plate 12, Fig. 15-16)

Sometimes individuals with nearly straight apices or very short spines were observed (Plate 12, Fig. 16). They fit drawing of West et al. (1923; Plate 129, Fig. 7), while their dimensions, according to Teiling (1967), fit formae majores.

Lg. 18.4-27.6 $\mu \mathrm{m}$, br. 18.4-25.3 $\mu \mathrm{m}$, isth. 4.6-6.9 $\mu \mathrm{m}$, sp. 1.1-2.3 $\mu \mathrm{m}$, lg.: br. 1.00-1.09 $\times$.

Occur.: 1rrr, 2rrr, 7rrr, 8rr, 9rir.

var. apiculatus (Bréb.) Teil. (Plate 12, Fig. 10)

Lg. 17.2-24.1 $\mu \mathrm{m}$, br. 17.2-23.0 $\mu \mathrm{m}$, isth. $5.7 \mu \mathrm{m}$, sp. 1.1-2.3 $\mu \mathrm{m}$, lg.: br. 0.93-1.07 $\times$.

Occur.: 7rrr, 8rrr, 9rr.

Staurodesmus dickiei (Ralfs) Lillier.

var. dickiei (Plate 13, Fig. 18)

Lg. 20.7-24.1 $\mu \mathrm{m}$, br. 18.4-24.1 $\mu \mathrm{m}$, isth. 6.9-8.0 $\mu \mathrm{m}$, sp. 2.3-3.4 $\mu \mathrm{m}$, lg.: br. 0.95-1.13 $\times$.

Occur.: 2rrr.

var. rhomboideus (W. et G. S. West) Lillier. (Plate 13, Fig. 19)

Cells with more flattened apices and wider open sinus than given by Teiling (1967). They correspond to specimens found by: Bicudo (1969), Mix (1970), Lind (1971) and Rủžička (1972).

Lg. 23.0-27.6 $\mu \mathrm{m}$, br. 25.3-29.9 $\mu \mathrm{m}$, isth. 6.9-9.2 $\mu \mathrm{m}$, sp. 5.7-6.9 $\mu \mathrm{m}$, lg.: br. 0.91-0.96 $\times$.

Occur.: 7rrr, 8rrr, 9rrr.

\section{Plate 12}

Fig. 1-2. Xanthidium antilopaeum var. antilopaeum fac. 2. Fig. 3-5. X. antilopaeum var. antilopaeum fac. 2, morpha. Fig. 6. X. antilopaeum var. antilopaeum fac. 3. Fig. 7. X. armatum var. armatum. Fig. 8. Staurodesmus patens var. patens. Fig. 9. Std. mamillatus var. mamillatus. Fig. 10. Std. dejectus var. apiculatus. Fig. 11-12. Std. o'mearae var. o'mearae fac. 3. Fig. 13. Std. o'mearae var. o'mearae fac. 2. Fig. 14. Std. connatus var. connatus. Fig. 15-16. Std. dejectus var. dejectus 
PLATE 12
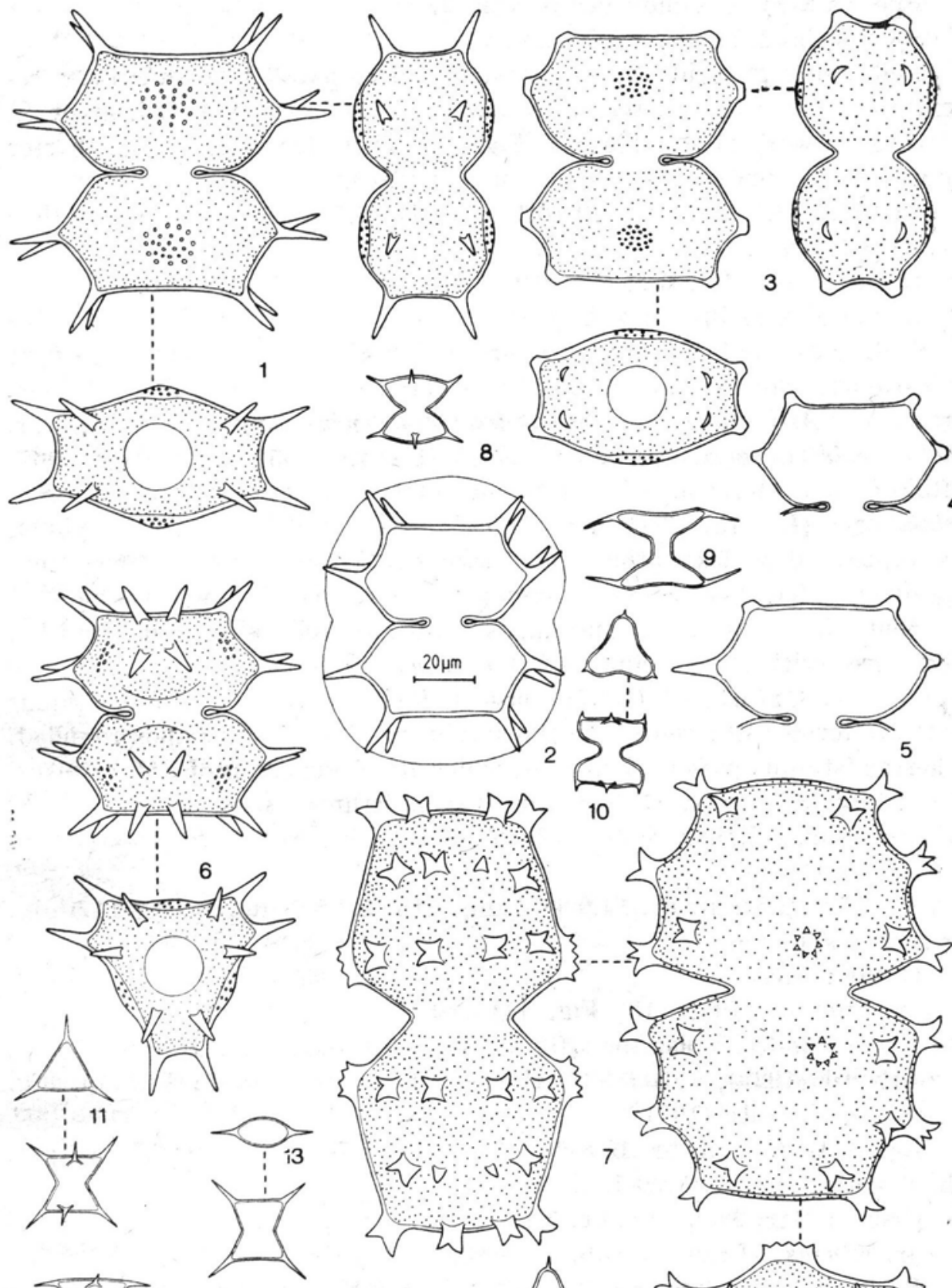

$\{-5-5]$
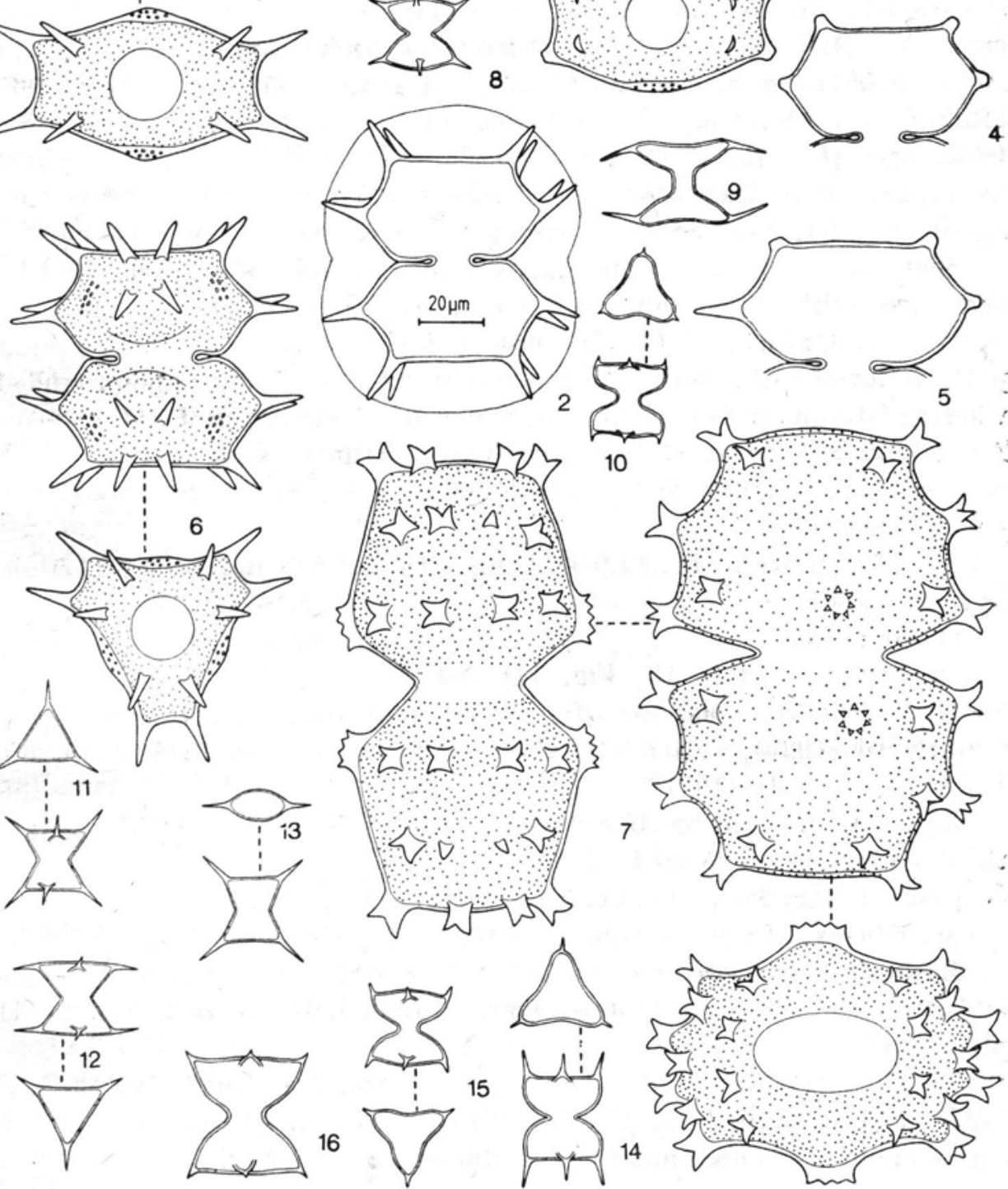

16

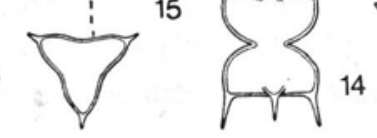
$\sqrt{3}$ 
Staurodesmus extensus (Anderss.) Teil.

Species very morphologically varied.

a) morpha 1 (Plate 13, Fig. 9)

Cells with straight apices, concave sides, parallel spines. They resemble the drawing of std. extensus var. joshuae (Gutw.) Teil. given in Teiling's work (1967; Plate 5, Fig. 23). They differ from it in shorter spines, more concave sides and rounded sinus.

Lg. 17.2-19.5 $\mu \mathrm{m}$, br. 14.9-16.1 $\mu \mathrm{m}$, isth. $5.7 \mu \mathrm{m}$, sp. 8.0-9.2 $\mu \mathrm{m}$, lg.: br. $1.15-1.21 \times$.

Oceur.: 3rrr, 4rr, 6rr, 7rr, 9rrr.

b) morpha 2 (Plate 13, Fig. 10)

Cells with slightly convex, depressed in the middle apices, concave sides and parallel spines. Semicells appearance fits the drawing and description of Arthrodesmus ( = Staurodesmus) extensus (Anderss.) Hirano var. malaccensis (Bernard) Hirano given by Hirano (1957b). Teiling (1967) affiliates the above-mentioned taxon holotype to the synonyms of $s t d$. triangularis (Lagerh.) Teil. var. triangularis, fac. 2. I consider this opinion as unjustified as description and drawing in Hirano's work differs from specimens identified as Std. triangularis.

Semicells shape in the specimens found, as well as spine distribution also agree with the specimen of Art. incus var. extensus, form given by Scott and Grönblad (1957; Plate 9, Fig. 13), but sinus and isthmus part are formed differently. In the specimens studied sinus apex rounded, whereas isthmus part is elongated, which fits description of Std. extensus. According to Scott, Grönblad (1957), isthmus part is cup-shaped, whereas sinus slightly notch which agrees with Std. indentatus (W. et G. S. West) Teil.

Lg. 17.2-21.8 $\mu \mathrm{m}$, br. 14.9-18.4 $\mu \mathrm{m}$, isth. 5.7-8.0 $\mu \mathrm{m}$, sp. 5.7-11.5 $\mu \mathrm{m}$, lg.: br. $1.00-1.29 \times$.

Occur.: $7 \mathrm{ec}$.

var. extensus (Plate 13, Fig. 11)

Typical individuals, they fit drawings in works of: Borge (1913), Grönblad (1920), Taft (1937) given in Teiling's (1967; Plate 5, Fig. 18) work, Hinode (1971) and others.

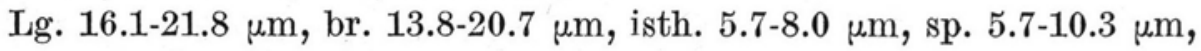
th. $6.9 \mu \mathrm{m}$, lg.: br. $1.00-1.17 \times$.

Occur.: 1rrr, 2rrr, 3rrr, 4r, 5rrr, 6e, 7rrr, 8ce, 9rrr.

var. retusus (Hirano) comb. nov. (Plate 13, Fig. 12)

Basion.: Arthrodesmus extensus (Anderss.) Hirano var. retusus Hirano (1957), Flora Desmidiarum Japonicarum, Biol. Lab. Kyoto Univ., p. 211, pl. $29: 38$.

Teiling (1967) includes this taxon in Std. indentatus (W. et G. S. West) Teil. I think it should be affiliated to Std. extensus as shape of the isthmus part and sinus fits description of the latter. 
Lg. 16.1-20.7 $\mu \mathrm{m}$, br. 13.8-16.1 $\mu \mathrm{m}$, isth. 4.6-6.9 $\mu \mathrm{m}$, sp. 4.6-6.9 $\mu \mathrm{m}$, th. $8.0 \mu \mathrm{m}$, lg.: br. 1.17-1.29 $\times$.

Occur.: 7e, 8rrr, 9rrr.

var. vulgaris (Eichl. et Racib.) Croas. (Plate 13, Fig. 13)

Messikommer (1971b) quotes specimens with longer spines.

Lg. 13.8-19.5 $\mu \mathrm{m}$, br. 11.5-16,1 $\mu \mathrm{m}$, isth. 4.6-8.0 $\mu \mathrm{m}$, sp. 2.3-4.6 $\mu \mathrm{m}$, lg.: br. $1.00-1.25 \times$.

Occur.: 4r, 6rr, 7rr, 9rrr.

Specimens of morpha 1 and 2, as well as var. retusus were the most abundant in planktonic samples in Grabicz A bog. Ecological conditions of this site differ from the other bogs (see p. 5). Probably, they favour development of such Std. extensus forms.

Individuals of morpha 1 and 2, as well as var. retusus were observed three-fold in the same samples. Therefore, one may suppose that differences between them are of no essential taxonomical value and lie in the intrapopulational variation.

Amongst individuals of the above species I encountered some specimens with only one small spine (Plate 13, Fig. 14).

Staurodesmus glabrus (Ehrenb. ex Ralfs) Teil.

var. glabrus (Plate 13, Fig. 7).

Lg. 18.4-21.8 $\mu \mathrm{m}$, br. 17.2-20.7 $\mu \mathrm{m}$, isth. 6.9-8.0 $\mu \mathrm{m}$, sp. 4.6-6.9 $\mu \mathrm{m}$, lg.: br. 0.89-1.13 $\times$.

Occur.: 2rrr, 4rr, 6rrr, 7rr, 8rrr, 9rrr.

var. debaryanus (Nordst.) Teil. (Plate 13, Fig. 5-6)

a) Individual appearance agrees with drawings of this variety in works of: Teiling (1967; Plate 14, Fig. 3) and Förster (1970), (Plate 13, Fig. 5).

Lg. 18.4-19.5 $\mu \mathrm{m}$, br. 16.1-17.2 $\mu \mathrm{m}$, isth. 5.7-6.9 $\mu \mathrm{m}$, sp. 5.7-6.9 $\mu \mathrm{m}$, lg.: br. 1.06-1.14 $\times$.

Occur.: 6rr, 7rr, 8rr, 9rrr.

b) morpha (Plate 13, Fig. 6)

Cells small with very short spines. Their shape and size similar to small specimens of Std. dickiei (Ralfs) Lillier. var. dickiei, but their ventral margin is less convex than dorsal margin.

Lg. 14.9-16.1 $\mu \mathrm{m}$, br. $14.9 \mu \mathrm{m}$, isth. $6.9 \mu \mathrm{m}$, sp. $2.3 \mu \mathrm{m}$, lg.: br. $1.00-$ $1.08 \times$.

Occur.: 7rrr.

var. limnophilus (Teil.) Teil. (Plate 13, Fig. 8)

Specimens with spines shorter than those given by Teiling (1967). Lg. 18.4-19.5 $\mu \mathrm{m}$, br. $18.4 \mu \mathrm{m}$, isth. $6.9 \mu \mathrm{m}$, sp. 10.3-11.5 $\mu \mathrm{m}$, lg.: br. $1.00-1.07 \times$.

\section{Occur.: 8rrr.}

Staurodesmus incus (Bréb. ex Ralfs) Teil.

var. incus f. incus (Plate 13, Fig. 1) 
Lg. 16.1-18.4 $\mu \mathrm{m}$, br. 13.8-16.1 $\mu \mathrm{m}$, isth. 6.9-9.2 $\mu \mathrm{m}$, sp. 10.3-11.5 $\mu \mathrm{m}$, th. $6.9-8.0 \mu \mathrm{m}$, lg.: br. $1.14-1.17 \times$.

Occur.: 1rrr, 2rrr, 4r, 6rrr, 9rrr.

f. minor (W. et G. S. West) Teil. (Plate 13, Fig. 2)

Lg. 13.8-14.9 $\mu \mathrm{m}$, br. 13.8-14.9 $\mu \mathrm{m}$, isth. 4.6-8.0 $\mu \mathrm{m}$, sp. $6.9 \mu \mathrm{m}$, th. $6.9 \mu \mathrm{m}$, lg.: br. $1.00 \times$.

Occur.: 4rrr, 9rrr.

? Staurodesmus indentatus (W. et G. S. West) Teil., morpha (Plate 13, Fig. 23)

Specimens smaller than those described in the relevant literature. Their shape agrees with the drawing of Std. indentatus in Teiling's (1967; Plate 7, Fig. 12) work, but slightly differs from that in work of Croasdale, Scott (1976). Specimens encountered by Croasdale and Scott and identified as Std. indentatus are characterized by apices very slightly elevated, horizontal spines and less widen sinus. The shape and size of individuals also fit Std. extensus, but isthmus part and sinus are formed differently. One cannot be sure which character to consider as more taxonomically valuable - size or shape of isthmus part and sinus. Some authors such as Scott, Grönblad (1957), Rủžička (1973a), Coesel, Hoogendijk (1975) and Lenzenweger (1977) all specimens with isthmus and sinus formed as in Std. indentatus, but with different distribution and size of spines classify as Std. extensus.

Therefore, I am not sure whereas the specimens encounterd are representatives of a small form of std. indentatus, or wherher they should be classified as Std. extensus.

Lg. 16.1-19.5 $\mu \mathrm{m}$, br. 13.9-17.2 $\mu \mathrm{m}$, isth. 4.6-5.7 $\mu \mathrm{m}$, sp. 4.6-9.2 $\mu \mathrm{m}$, lg.: br. 1.13-1.17 $\times$.

Occur.: 7rr.

Staurodesmus isthmosus (Heimerl) Croas.

var. isthmosus (Plate 13, Fig. 15-17)

In some specimens apices slightly depressed, spines somewhat divergent (Plate 13, Fig. 16). In Biały Lug B bog a specimen with an additional spine at a base of one semicell was encountered (Plate 13, Fig. 17).

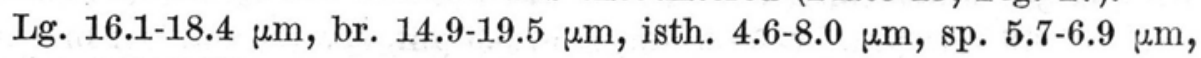
lg.: br. 0.93-1.07 $\times$.

Occur.: 1rrr, 2rr, 3rrr, 4c, 6r, 7rr, 8r, 9rr.

Staurodesmus mamillatus (Nordst.) Teil.

var. mamillatus (Plate 12, Fig. 9)

Lg. 18.4-23.0 $\mu \mathrm{m}$, br. 18.4-21.8 $\mu \mathrm{m}$, isth. 5.7-6.9 $\mu \mathrm{m}$, sp. 9.2-11.5 $\mu \mathrm{m}$, lg.: br. $0.84-1.25 \times$.

Occur.: 8rir, 9rrr.

Staurodesmus mucronatus (Ralfs ex Bréb.) Croas.

var. mucronatus (Plate 13, Fig. 20) 


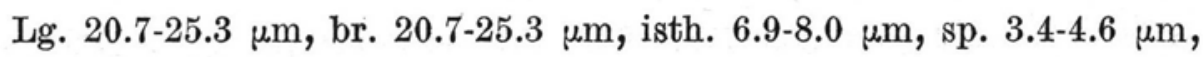
lg.: br. 0.91-1.00 $\times$.

Occur.: 6rrr.

var. subtriangularis (W. et G. S. West) Croas. (Plate 13, Fig. 21)

A shape and size of individuals are very close to, or completely agree with drawings of Std. mucronatus (= Staurastrum) var. subtriangularis in works of: Borge (1913), Teiling (1967; Plate 18, Fig. 13) and Hirano (1968). Apices are more convex than in specimens described by West et al. (1923) and Hirano (1972). They also fit drawings of Staurodesmus (= Staurastrum) megacanthus (Lund.) Thunm. var. megacanthus given by Scott, Prescott (1961) and Förster (1970). Teiling (1967) classifies a drawing in Scott, Prescott's (1961) work both to the typical variety of Std. megacanthus and to Std. mucronatus var. subtriangularis (p. 553 and 570).

Appearance and size of the specimens encountered differ from typical individuals of Std. megacanthus, described by: West et al. (1923), Hirano (1959b), Teiling (1967) and others. Cells are smaller, spines shorter and less massive.

I think that the specimens encountered have more characters in common with those of Std. mucronatus var. subtriangularis, than with those of Std. megacanthus var. megacanthus.

Lg. 25.3-26.4 $\mu \mathrm{m}$, br. $25.3 \mu \mathrm{m}$, isth. $6.9 \mu \mathrm{m}$, sp. $3.4-4.6 \mu \mathrm{m}$, lg.: br. 1.00-1.04 $\times$.

Occur.: 7rr.

Staurodesmus o'mearae (Arch.) Teil.

var. o'mearae (Plate 12, Fig. 11-13)

a) fac. 2 (Plate 12, Fig. 13)

Lg. 13.8-16.1 $\mu \mathrm{m}$, br. 11.5-13.8 $\mu \mathrm{m}$, isth. 8,0-9.2 $\mu \mathrm{m}$, sp. 6.9-8.0 $\mu \mathrm{m}$, lg.: br. 1.17-1.20 $\times$.

Occur.: 6cc.

b) fac. 3 (Plate 12, Fig. 11-12)

Amongst individuals of fac. 3 specimens with parallel spines (Plate 12, Fig. 12) were encountered. They agree with a drawing of Staurastrum o'mearae f. parallela Ström. from Hirano's (1959b) work. Teiling (1967) considers the taxon mentioned above as a synonym of the typical variety.

Lg. 14.9-18.4 $\mu \mathrm{m}$, br. 13.8-18.4 $\mu \mathrm{m}$, isth. 6.9-9.2 $\mu \mathrm{m}$, sp. 4.6-6.9 $\mu \mathrm{m}$, lg.: br. 0.88-1.08 $\times$.

Occur.: 4r, 6rr, 7rrr, 8rrr, 9rrir.

Staurodesmus patens (Nordst.) Croas.

var. patens (Plate 12, Fig. 8)

Lg. $18.4 \mu \mathrm{m}$, br. $17.2 \mu \mathrm{m}$, isth. $6.9 \mu \mathrm{m}$, sp. $3.4 \mu \mathrm{m}$, lg.: br. $1.07 \times$. Occur.: 7rrr. 
? Staurodesmus quiriferus (W. et G. S. West) Teil., morpha (Plate 13, Fig. 3-4)

In specimens apices are straight or slightly convex, spines short, isthmus elongated, sinus widely open, rounded at the extremity. Cell size and shape similar to those of Arthrodesmus quiriferus W. et G. S. West var. brevispinis Okada from Hirano's (1957b) drawing. They differ from var. brevispinis in an apex shape, which in this variety and in other taxa of Staurodesmus (= Arthrodesmus) quiriferus are concave.

Specimen appearance also agrees with drawings of Arthrodesmus incus (Bréb.) Hass. ex Ralfs var. incus f. minor W. et G. S. West from Wests' (1912) work (Plate 113, Fig. 16, 18). The only difference lies in the fact that isthmus is more elongated and sinus with distinctly rounded apex in the encountered specimens. In specimens identified by the author as Std. incus var. incus f. minor, which fit drawings of present taxon in works of West, West (1912; Plate 113, Fig. 17) and Lenzenweger (1967), isthmus is not elongated and sinus acuminate at the apex. I suppose that drawings of Art. incus var. incus f. minor by Wests represent two different taxa.

It has not been learned yet which morphological character studied isthmus and sinus shape or apices shape - is more stabile thus more valuable in the taxonomy of both taxa (Std. incus and Std. quiriferus). I assumed the first one as the more important and therefore, I identify specimens as Std. quiriferus.

Hirano in Yamagishi, Hirano's (1973) work distingushes within Arthrodesmus (= Staurodesmus) quiriferus a new taxon - var. minus. Unfortunately, he does not give any drawing or more detailed description. Encountered specimens might be treated as representatives of this taxon.

Lg. 11.5-14.9 $\mu \mathrm{m}$, br. 10.3-13.8 $\mu \mathrm{m}$, isth. 4.6-5.7 $\mu \mathrm{m}$, sp. 3.4-8.0 $\mu \mathrm{m}$, th. $3.4-5.7 \mu \mathrm{m}$, lg.: br. 1.00-1.20 $\times$.

Occur.: $4 \mathrm{e}, 6 \mathrm{c}$.

Staurodesmus ralfsii (W. West) comb. nov.

Basion.: Arthrodesmus ralfsii $\mathrm{W}$. West (1892), A contribution to the freshwater algae of West Ireland, J. Linn. Soc. Bot., 29, p. 168.

var. ralfsii, morpha (Plate 13, Fig. 22)

I think the present taxon should be treated as an unit of the species rank, given it by W. West and not as a variety of Std. incus according to Teiling (1967)

In the specimens encountered semicells are trapeziform, their sides are convergent, apices convex, spines short slightly divergent. Size and setting of spines differ from those in the drawings of this taxon in work of West, West (1912). The shape of specimens encountered totally agrees with a drawing of Arthrodesmus incus (Bréb.) Hass. ex Ralfs f. Ralfs from Turner's (1892) (Plate 12, Fig. 6a). Teiling (1967) considers this taxon as a synonym of Staurodesmus (=Arthrodesmus) incus 
var. ralfsii. However, West, West (1912) do not quote Turner's work in the lists of synonyms of Arthrodesmus incus (Bréb.) Hass. ex Ralfs var. ralfsii W. et G. S. West. Cells also fit the drawing of Arthrodesmus incus f. isthmosa Heimerl with short spines given by Huber-Pestalozzi (1928; Plate 13, Fig. 2).

Lg. 16.1-18.4 $\mu \mathrm{m}$, br. 12.6-14.9 $\mu \mathrm{m}$, isth. 5.7-6.9 $\mu \mathrm{m}$, sp. 3.4-8.0 $\mu \mathrm{m}$, lg.: br. 1.23-1.28 $\times$.

Occur.: 6rr.

Staurodesmus spencerianus (Mask.) Teil.

var. spencerianus (Plate 13, Fig. 24-25)

a) Typical specimens, their character agrees with data from relevant literature (Plate 13, Fig. 24)

Lg. 17.2-23.0 $\mu \mathrm{m}$, br. 16.1-20.7 $\mu \mathrm{m}$, isth. $6.9 \mu \mathrm{m}$, sp. 8.0-9.2 $\mu \mathrm{m}, \mathrm{lg}$.: br. 1.06-1.11 $\times$.

Occur.: 2rr, 3rr, 4c, 5rrr, 6ec, 7rrr, 8ce, 9rr.

b) morpha (Plate 13, Fig. 25)

Individuals differ from typical specimens in longer and parallel spines. Cells shape similar to that described by Krieger (1933a; Plate 14, Fig. 11) as Staurastrum dejectum Bréb. ex Bréb., form and which has been described by Teiling (1967) as Std. spencerianus - typical variety. If compared, in specimens encountered sides and apices are more convex.

Lg. $20.7 \mu \mathrm{m}$, br. 18.4-19.5 $\mu \mathrm{m}$, isth. 5.7-6.9 $\mu \mathrm{m}$, sp. 11.5-13.8 $\mu \mathrm{m}$, lg.: br. 1.06-1.12 $\times$.

Occur.: 8rr.

\section{Staurodesmus triangularis (Lagerh.) Teil.}

In the area studied the species is highly morphologically varied. The following taxa and morphological groups have been distinguished:

morpha (Plate 13, Fig. 26)

Cells small, with markedly elongated isthmus and convergent spines. A shape of specimens close to that in drawings of Arthrodesmus (= Staurodesmus) triangularis Lagerh. in Růžička's (1972) work. They differ from it in elongated isthmus. Isthmus shape resembles that of Std. cuspidatus (Bréb. ex Bréb.) Teil., but encountered specimens are smaller with concave apices and semicells triangular in face view.

Lg. 12.6-13.8 $\mu \mathrm{m}$, br. $11.5 \mu \mathrm{m}$, isth. $3.4 \mu \mathrm{m}$, sp. 4.6-6.9 $\mu \mathrm{m}$, lg.: br. 1.10-1.20 $\times$.

Occur.: 7rr, 8rrr, 9rrr.

var. triangularis (Plate 13, Fig. 27-29)

a) Cells fit the described specimens of typical variety with the exception of spines shorter than those given by: West, West (1912), Teiling (1967), Thomasson (1973), and Lenzenweger (1974) (Plate 13, Fig. 27-28).

Lg. 18.4-21.8 $\mu \mathrm{m}$, br. 16.1-20.7 $\mu \mathrm{m}$, isth. 4.6-6.9 $\mu \mathrm{m}$, sp. 6.9-8.0 $\mu \mathrm{m}$, th. $6.9 \mu \mathrm{m}, \mathrm{lg} .:$ br. $1.05-1.14 \times$. 
Occur.: 2rrr, 7rrr, 9rr.

b) morpha (Plate 13, Fig. 29)

Cells small, without elongated isthmus part. Their size slightly above upper limit of dimensions given by Teiling (1967) for Std. triangularis, formae minores.

Lg. 12.6-13.8 $\mu \mathrm{m}$, br. 11.5-13.8 $\mu \mathrm{m}$, isth. $4.6 \mu \mathrm{m}$, sp. $4.6-5.7 \mu \mathrm{m}$, lg.: br. $1.00-1.10 \times$.

Occur.: 6rrr, 7rr.

var. subhexagonus (W. et G. S. West) Teil. (Plate 13, Fig. 30)

Lg. 11.5-16.1 $\mu \mathrm{m}$, br. 10.3-14.9 $\mu \mathrm{m}$, isth. 4.6-6.9 $\mu \mathrm{m}$, sp. 2.3-3.4 $\mu \mathrm{m}$, th. 5.7-6.9 $\mu \mathrm{m}, \mathrm{lg} .:$ br. 1.08-1.22 $\times$.

Occur.: 2rrr.

\section{Genus: Cosmoastrum Palamar-Mordvintseva 1976}

Cosmoastrum alternans (Bréb.) Pal.-Mordv.

var. alternans (Plate 13, Fig. 38)

Specimens with straight apices, widely open sinus and densely granulate cell wall. They fit drawings in works of: Grönblad (1960), Grönblad, Croasdale (1971) and Růžička (1973a).

Lg. 21.8-25.3 $\mu \mathrm{m}$, br. 24.1-27.6 $\mu \mathrm{m}$, isth. 9.2-10.3 $\mu \mathrm{m}$, lg.: br. 0.86$0.95 \times$.

Occur.: 7rrr, 9rrr.

Cosmoastrum brebissonii (Arch.) Pal.-Mordv.

var. brebissonii (Plate 13, Fig. 40)

Lg. 32.2-48.3 $\mu \mathrm{m}$, br. 32.2-46.0 $\mu \mathrm{m}$, isth. 11.5-13.8 $\mu \mathrm{m}$, sp. 2.3-3.4 $\mu \mathrm{m}$, lg.: br. 1.00-1.07 $\times$.

Occur.: 1rrr, 7rrr, 9rirr.

Cosmoastrum gladiosum (Turn.) Pal.-Mordv.

var. gladiosum (Plate 13, Fig. 41)

Lg. 33.3-35.6 $\mu \mathrm{m}$, br. 28.7-31.0 $\mu \mathrm{m}$, isth. 9.2-11.5 $\mu \mathrm{m}$, sp. 4.6-5.7 $\mu \mathrm{m}$, lg.: br. 1.11-1.15 $\times$.

Occur.: 2rrrr, 8rrri, 9rrr.

Cosmoastrum hirsutum (Ehrenb. ex Bréb.) Pal.-Mordv.

var. hirsutum (Plate 13, Fig. 37)

Lg. 34.5-41.4 $\mu \mathrm{m}$, br. 27.6-33.3 $\mu \mathrm{m}$, isth. 8.0-13.8 $\mu \mathrm{m}$, sp. 1.1-2.3 $\mu \mathrm{m}$, lg.: br. 1.15-1.28 $\times$.

Occur.: 1rrr, 2rrr, 3rrr, 4r, 5rr, 6r, 7rrr, 8rr, 9rr.

Cosmoastrum hystrix (Ralfs) Pal.-Mordv.

var. pannonicum (Lütkem.) comb. nov. (Plate 14, Fig. 9)

Basion.: Staurastrum hystrix Ralfs var. pannonicum Lütkemüller (1900), Desmidiaceen aus der Umgebung des Millstättersees in Kärnten, Verh. k. k. zool.-bot. Ges. Wien, 50, p. 80, pl. 1 : 52-53.

Spines longer less curved, besides it fits Grönblad's (1920) drawing. 
Lg. $21.8-25.3 \mu \mathrm{m}$, br. 18.4-23.0 $\mu \mathrm{m}$, isth. $9.2 \mu \mathrm{m}$, sp. $5.7 \mu \mathrm{m}$, lg.: br. 1.10-1.18 $\times$.

Occur.: 1rrr, 2rrr, 3rrr, 6rrr.

Cosmoastrum lapponicum (Schmidle) Pal.-Mordv. comb. nov.

Basion.: Staurastrum punctulatum Bréb. var. muricatiforme Schmidle f. lapponica Schmidle (1898), Über einige von Knut Bohlin in Pite Lappmark und Vesterbotten gesammelten Süsswasseralgen, Bih. K. Sv. Vet. Akad. Handl., 24, p. 57, pl. $3: 5$.

Cosmoastrum lapponicum (Șchmidle) Palamar-Mordvintseva 1982, p. 94 and 579; combination not validly published, Art. 33 Int. Code.

var. lapponicum (Plate 13, Fig. 46)

Lg. $31.0-33.3 \mu \mathrm{m}$, br. $32.2 \mu \mathrm{m}$, isth. $9.2-11.5 \mu \mathrm{m}$, lg.: br. 0.96-1.03 $\times$.

Occur: 4rrr, 9rrr.

Cosmoastrum muricatiforme (Schmidle) comb. nov.

Basion.: Staurastrum muricatiforme Schmidle (1895), Beiträge zur alpinen Algenflora, Österr. bot. Zeitschr., 45, p. 162, pl. 16 : 14-15.

var. muricatiforme (Plate 13, Fig. 35)

A shape of specimens and cell wall sculpture agree with description of this taxon in works of: Migula (1907) and West, West (1912), but totally differs from that of Hirano (1968). Size of most individuals exceeds dimension upper limit given in the above-mentioned works. They fit specimens described by Rủžička (1954).

I think that species rank given by Schmidle (1895; after West, West 1912 ) should be maintained. It may be also treated as a variety of Cosmoastrum (= Staurastrum) muricatum. It definitely should not be considered as a variety of Cosmoastrum (= Staurastrum) punctulatum, some years after taxon description Schmidle (1898; after West, West 1912) affiliated it in this way.

Both taxa differ morphologically:

1) In Cos. muricatiforme semicells are elliptic to nearly semicircular, in Cos. punctulatum - nearly rhomboid-elliptic;

2) Sinus open in both taxa, but in Cos. murcatiforme acute-angle of 10-30 ${ }^{\circ}$, while in Cos. punctulatum - of about $70^{\circ}$;

3) In Cos. muricatiforme cell wall is coarsely granulate, in Cos. punctulatum - finely granulate.

Lg. 34.5-50.6 $\mu \mathrm{m}$, br. 29.9-43.7 $\mu \mathrm{m}$, isth. 9.2-16.1 $\mu \mathrm{m}$, lg. : br. 1.05-1.31 ×.

Occur.: 1e, 2rr, 3rr, 4e, 5r, 6rr, 7rrr, 8rr, 9rrr.

Cosmoastrum muricatum (Bréb. ex Ralfs) Pal.-Mordv.

var. muricatum (Plate 13, Fig. 36)

Förster (1970) classifies the taxon present as a variety of Staurastrum hirsutum Ehrenb. ex Bréb.

Lg. 39.1-48.3 $\mu \mathrm{m}$, br. 32.2-43.7 $\mu \mathrm{m}$, isth. 11.5-16.1 $\mu \mathrm{m}$, lg.: br. 1.06$1.29 \times$.

Occur.: 1rrr, 2rrr, 3rrr, 4r, 5r, 6r, 7rrr, 8r, 9rrr. 
Cosmoastrum muticum (Bréb. ex Bréb.) Pal.-Mordv.

Basion.: Staurastrum muticum Bréb. ex Brébisson in Ralfs (1848), The British Desmidieae, London, p. 125, pl. 21:4.

var. muticum (Plate 13, Fig. 31)

Lg. 20.7-21.8 $\mu \mathrm{m}$, br. 20.7-23.0 $\mu \mathrm{m}$, isth. 5.7-6.9 $\mu \mathrm{m}$, lg.: br. 0.95-1.00 $\times$.

Occur.: 7rrr.

Cosmoastrum orbiculare (Ralfs ex Ralfs) Pal.-Mordv. comb. nov.

Basion.: Staurastrum orbiculare Ralfs ex Ralfs (1848), The British Desmidieae, London, p. 125, pl. $21: 5$.

Cosmoastrum orbiculare (Ralfs) Palamar-Mordvintseva 1982, p. 83 and 577; combination not validly published, Art. 33 Int. Code.

var. depressum (Roy et Biss.) Pal.-Mordv. (Plate 13, Fig. 33)

Cells less flattened than those described in the relevant literature.

Lg. $26.4 \mu \mathrm{m}$, br. $24.1 \mu \mathrm{m}$, isth. 6.9-8.0 $\mu \mathrm{m}$, lg.: br. $1.10 \times$.

Occur.: 7rrr, 9rrr.

Cosmoastrum punctulatum (Bréb.) Pal.-Mordv.

var. punctulatum (Plate 13, Fig. 44)

Lg. 25.3-29.9 $\mu \mathrm{m}$, br. 23.0-27.6 $\mu \mathrm{m}$, isth. 9.2-11.5 $\mu \mathrm{m}$, lg.: br. 1.04$1.18 \times$.

Occur.: 3c, 6ce, 7rrr, 9rrr.

var. kjellmanii (Wille) Pal.-Mordv. (Plate 13, Fig. 45)

Basion.: Staurastrum kjellmanii Wille (1879), Ferskvandsalger fra Novaja Semlja samlede af Dr F. Kjellman paa Nordenskiölds Expedition 1875, Öfvers. K. Vet.-Akad. Förh., 5, p. 50, pl. 13: 50-53.

\section{Plate 13}

Fig. 1. Staurodesmus incus var. incus f. incus. Fig. 2. Std. incus var. incus f. minor. Fig. 3-4. ? Std. quiriferus, morpha. Fig. 5. Std. glabrus var. debaryanus. Fig. 6. Std. glabrus var. debaryanus, morpha. Fig. 7. Std. glabrus var. glabrus. Fig. 8. Std. glabrus var. limnophilus. Fig. 9. Std. extensus, morpha 1. Fig. 10. Std. extensus, morpha 2. Fig. 11. Std. extensus var. extensus. Fig. 12. Std. extensus var. retusus. Fig. 13. Std. extensus var. vulgaris. Fig. 14. Std. extensus (reduced form). Fig. 15-17. Std. isthmosus var. isthmosus. Fig. 18. Std. dickiei var. dickiei. Fig. 19. Std. dickiei var. rhomboideus. Fig. 20. Std. mucronatus var. mucronatus. Fig. 21. Std. mucronatus var. subtriangularis. Fig. 22. Std. ralfsii var. ralfsii, morpha. Fig. 23. ? Std. indentatus, morpha. Fig. 24. Std. spencerianus var. spencerianus. Fig. 25. Std. spencerianus var. spencerianus, morpha. Fig. 26. Std. triangularis, morpha. Fig. 27-28. Std. triangularis var. triangularis. Fig. 29. Std. triangularis var. triangularis, morpha. Fig. 30. Std. triangularis var. subhexagonus. Fig. 31. Cosmoastrum muticum var. muticum. Fig. 32. Cos. wellipticum var. wellipticum. Fig. 33. Cos. orbiculare var. depressum. Fig. 34. Cos. turgescens var. sparsigranulatum. Fig. 35. Cos. muricatiforme var. muricatiforme. Fig. 36. Cos. muricatum var. muricatum. Fig. 37. Cos. hirsutum var. hirsutum. Fig. 38. Cos. alternans var. alternans. Fig. 39. Cos. scabrum var. scabrum. Fig. 40. Cos. brebissonii var. brebissonii. Fig. 41. Cos. gladiosum var. gladiosum. Fig. 42. Cos. teliferum var. teliferum. Fig. 43. Cos. teliferum var. pecten. Fig. 44. Cos. punctulatum var. punctulatum. Fig. 45. Cos. punctulatum var. kjellmanii. Fig. 46. Cos. lapponicum var. lapponicum. Fig. 47. Raphidiastrum avicula var. avicula, morpha 
PLATE 13
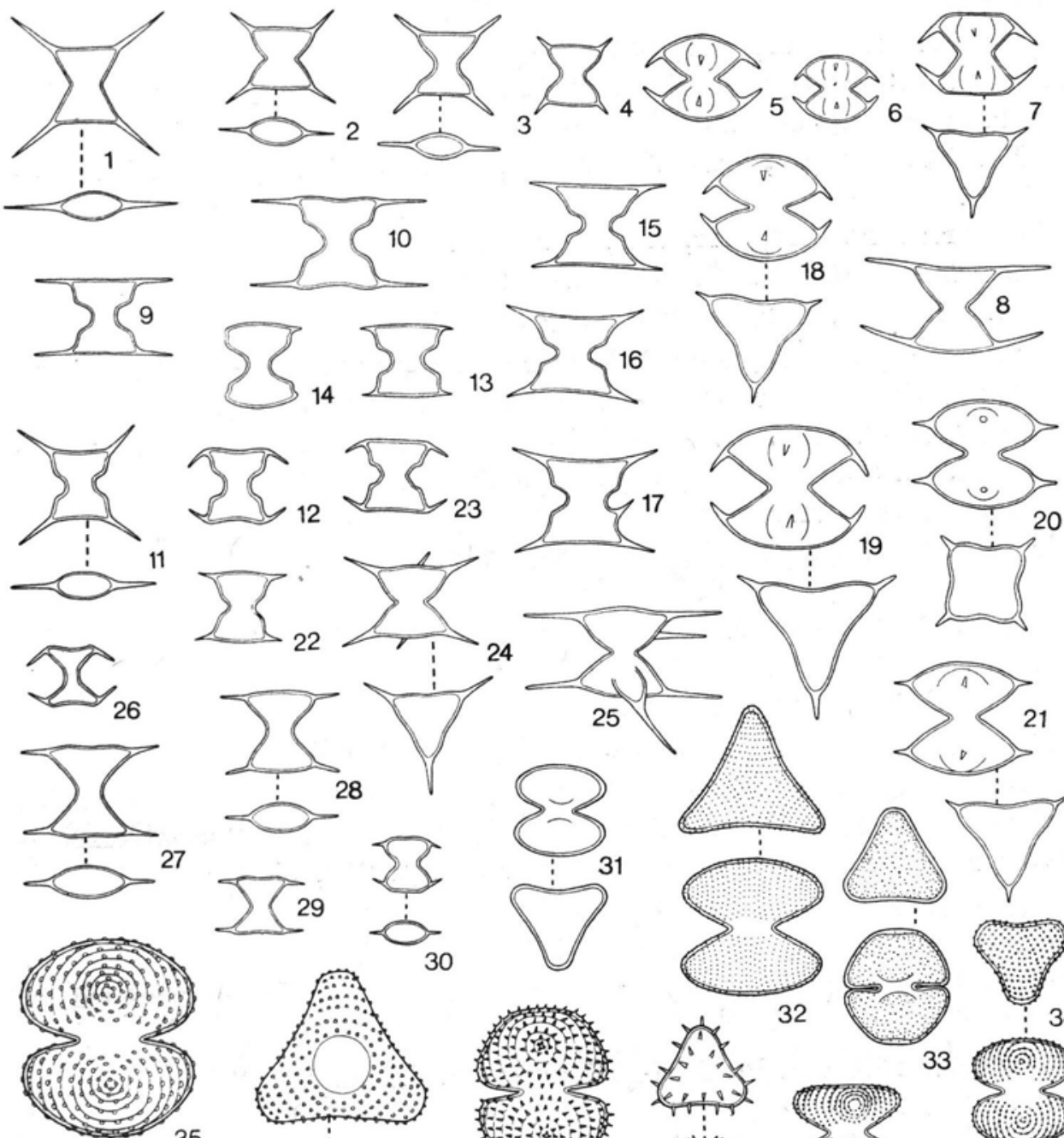

\section{9}
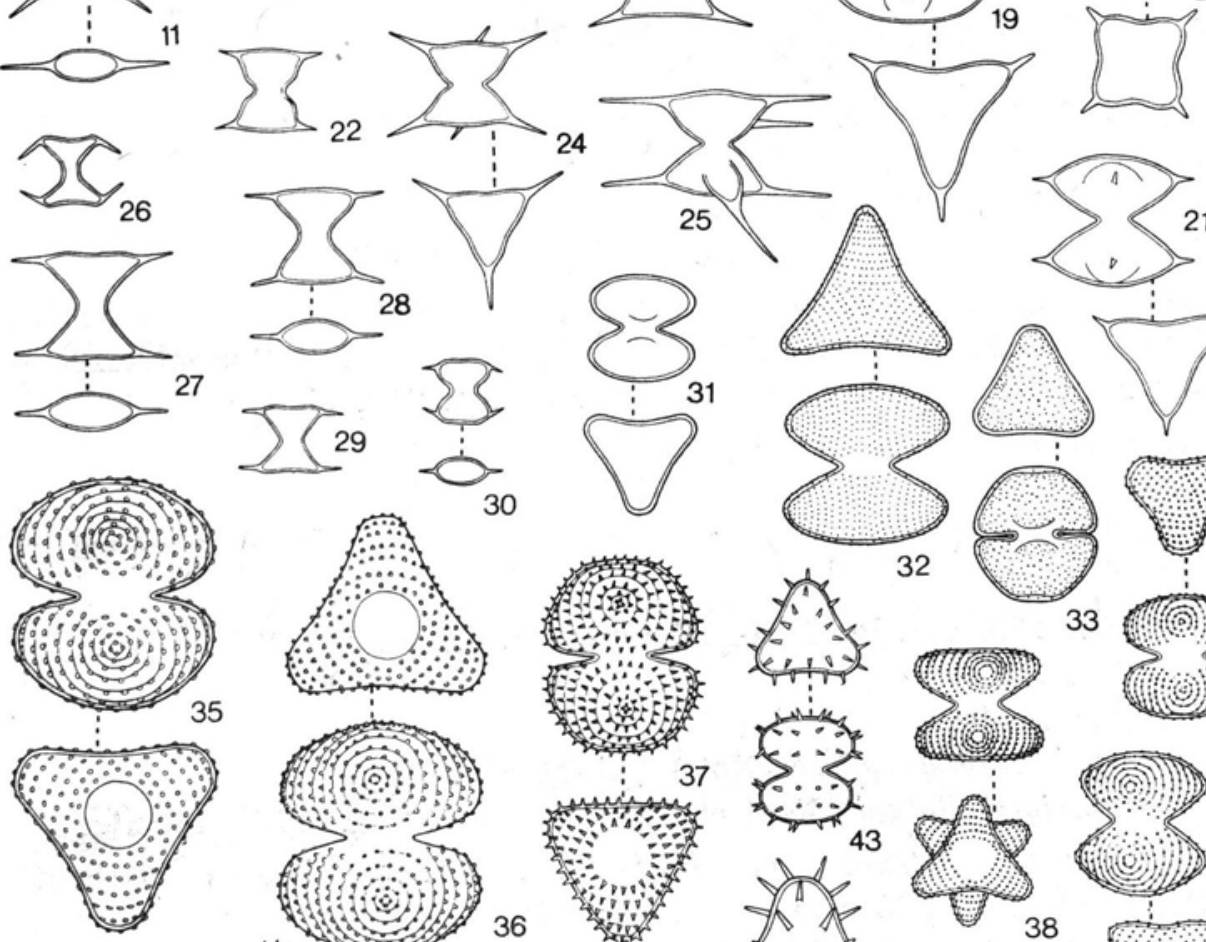

5
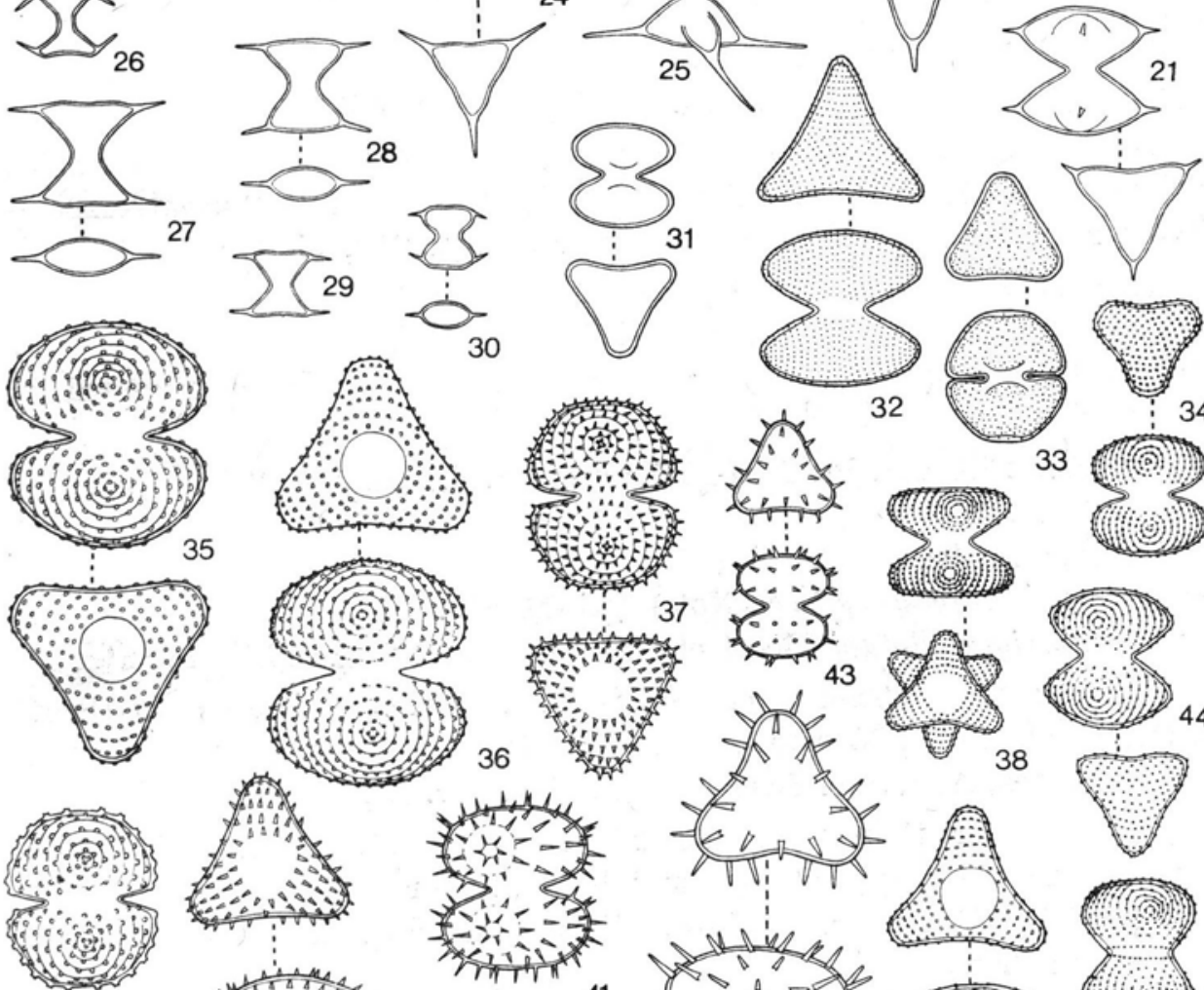

: $\therefore \therefore==$

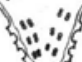
39
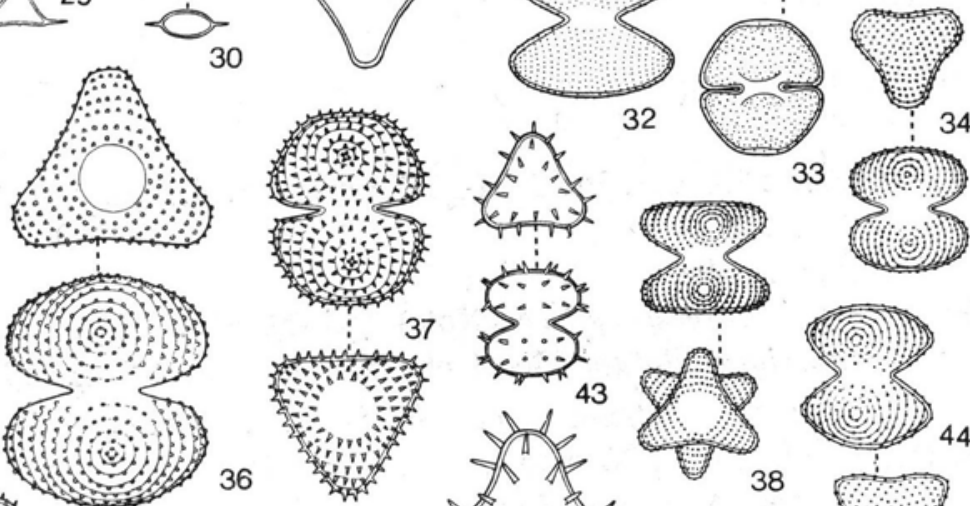

37

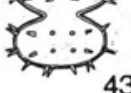

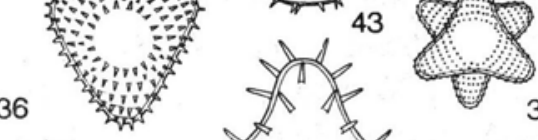
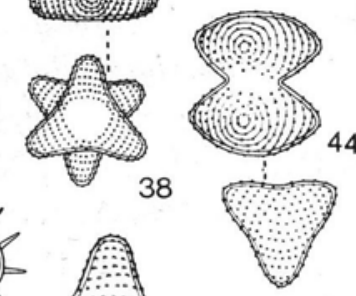

$7=019$

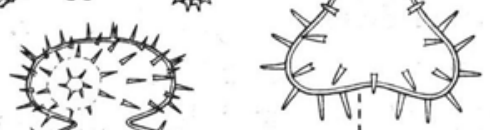

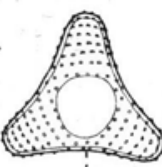

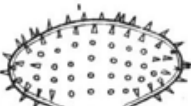

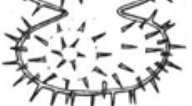

41 on is बालm $\gg$ (1)

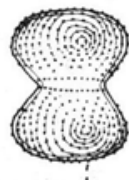
S.... 我: 40 24ifitios 47 \& 42

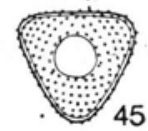




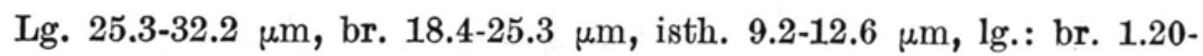
$1.44 \times$.

Occur.: 1rrr, 2rrr, 4rrr, 8rrr.

Cosmoastrum scabrum (Bréb.) Pal.-Mordv.

var. scabrum (Plate 13, Fig. 39)

Lg. 33.3-35.6 $\mu \mathrm{m}$, br. $31.0-32.2 \mu \mathrm{m}$, isth. 11.5-14.9 $\mu \mathrm{m}$, lg.: br. 1.07$1.15 \times$.

Occur.: 2rrr, 3rrr, 6rrr, 7rrr, 8rrr, 9rrr.

Cosmoastrum setigerum (Cleve) Pal.-Mordv.

var. setigerum, morpha (Plate 14, Fig. 1)

Angular spines only slightly longer and more massive than the others. Similar individuals are described by Scott, Prescott (1961).

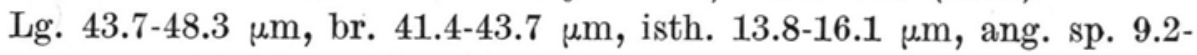
$10.3 \mu \mathrm{m}$, sp. 8.0-9.2 $\mu \mathrm{m}$, lg.: br. 1.06-1.07 $\times$.

Occur.: 7rrr, 9rrr.

Cosmoastrum teliferum (Ralfs) Pal.-Mordv.

var. teliferum (Plate 13, Fig. 42)

Lg. 39.1-41.4 $\mu \mathrm{m}$, br. 34.5-37.9 $\mu \mathrm{m}$, isth. 12.6-14.9 $\mu \mathrm{m}$, sp. 5.7-6.9 $\mu \mathrm{m}$, lg.: br. 1.06-1.13 $\times$.

Occur.: 2rrr, 7rrr, 8rir, 9rrr.

var. pecten (Perty) comb. nov. (Plate, 13, Fig. 43)

Basion.: Phycastrum pecten Perty (1852), Zur Kenntniss kleinster Lebensformen nach Bau, Funktionen, Systematik, mit Specialverzeichniss der in der Schweiz beobachteten, Bern, p. 210, pl. 16: 32.

Specimens fit some of Grönblad's (1945) drawings (Plate 14, Fig. 295), but differ from other (Plate 14, Fig. 294) and from those of Förster (1972). In the specimens encountered cell sides are more rounded in a frontal view.

Lg. 21.8-23.0 $\mu \mathrm{m}$, br. 18.4-19.5 $\mu \mathrm{m}$, isth. $6.9 \mu \mathrm{m}$, sp. $2.3 \mu \mathrm{m}$, lg.: br. $1.18-1.25 \times$.

Occur.: $7 \mathrm{e}, 9 \mathrm{r}$.

Cosmoastrum turgescens (De Not.) Pal.-Mordv.

var. sparsigranulatum (Scott et Grönbl.) comb. nov. (Plate 13, Fig. 34)

Basion.: Staurastrum turgescens De Not. var. sparsigranulatum Scott et Grönblad (1957), New and interesting Desmids from the Southeastern United States, Acta Soc. Scien. Fenn., Nov. Ser. B., 2, 8, p. 48, pl. 25: 8.

Cells smaller than those described by Scott, Grönblad (1957), but they fit some specimens found by Förster (1970).

Lg. 24.1-25.3 $\mu \mathrm{m}$, br. $23.0 \mu \mathrm{m}$, isth. 9.2-10.3 $\mu \mathrm{m}$, lg.: br. 1.05-1.10 $\times$.

Occur.: 2rrr.

Cosmoastrum wellipticum (Wille) comb. nov. et nom. nov.

Basion.: Staurastrum bieneanum Rabenh. var. ellipticum Wille (1879), Ferskvandsalger fra Novaja Semlja samlede af Dr F. Kjellman paa Nor- 
denskiölds Expedition 1875, Öfvers K. Vet.-Akad. Förh., 5 p. 50, pl. 13: 49.

var. wellipticum (Plate 13, Fig. 32)

Lg. $32.2-36.8 \mu \mathrm{m}$, br. $31.0-33.3 \mu \mathrm{m}$, isth. 9.2-11.5 $\mu \mathrm{m}$, lg.: br. 0.97$1.14 \times$.

Occur.: 7rrr, 8rrr, 9rrr.

Genus: Raphidiastrum (Turner) Palamar-Mordvintseva 1976

Raphidiastrum avicula (Bréb.) Pal.-Mordv.

var. avicula, morpha (Plate 13, Fig. 47)

Cells shape and length-breadth ratio differ from those of var. avicula given by West et al. (1923), Hirano (1959b, 1969), Förster (1970) and Coesel (1979b), but they agree with those of var. subarcuatum (Wolle) Pal.-Mordv. Grönblad (1948) stresses an important taxonomical character differentiating both mentioned above taxa i.e. cell wall sculpture, which has been omitted in the work of West et al. (1923). In var. avicula it is markedly granulate, in var. subarcuatum - verrucose.

In the encountered specimens the cell wall was granulate. Therefore, in spite of differences they were classified as var. avicula. Found individuals fit drawings of var. avicula in works of: Migula (1907, 1911), Thomasson (1972) and Růžička (1973a).

Lg. 23.0-24.1 $\mu \mathrm{m}$, br. $25.3 \mu \mathrm{m}$, isth. 6.9-8.0 $\mu \mathrm{m}$, sp. 3.4-4.6 $\mu \mathrm{m}$, lg.: br. $0.91-0.95 \times$.

\section{Occur.: 7rrr.}

Raphidiastrum denticulatum (Näg.) Pal.-Mordv.

var. denticulatum (Plate 14, Fig. 10)

The specimens smaller than those described by Migula (1907, 1911), Gutwiński (1909), West et al. (1923) and Hirano (1959b),

Lg. 20.7-23.0 $\mu \mathrm{m}$, br. 20.7-23.0 $\mu \mathrm{m}$, isth. 6.9-9.2 $\mu \mathrm{m}$, sp. $2.3 \mu \mathrm{m}, \mathrm{lg}$.: br. 0.95-1.00 $\times$.

Occur.: 4rrr, 8rrr, 9rrr.

Raphidiastrum granulosum (Ehrenb. ex Ralfs) Pal.-Mordv.

var. granulosum (Plate 14, Fig. 8)

Cell wall sculpture slightly differs from that in the drawing of West, West (1912). Each angle only with four rows of concentric granules.

Lg. 28.7-29.9 $\mu \mathrm{m}$, br. 26.4-27.6 $\mu \mathrm{m}$, isth. 9.2-10.3 $\mu \mathrm{m}$, sp. 1.1-2.3 $\mu \mathrm{m}$, lg.: br. 1.08-1.09 $\times$.

Occur.: 6rrr.

Raphidiastrum lunatum (Ralfs) Pal.-Mordv.

var. lunatum (Plate 14, Fig. 7)

Lg. 34.5-40.2 $\mu \mathrm{m}$, br. 34.5-42.5 $\mu \mathrm{m}$, isth. 13.8-14.9 $\mu \mathrm{m}$, sp. 3.4-4.6 $\mu \mathrm{m}$, lg.: br. $0.95-1.00 \times$.

Occur.: 7rrr, 9rrr. 
Raphidiastrum simonyi (Heimerl) Pal.-Mordv.

var. simonyi (Plate 14, Fig. 2)

Lg. 19.5-25.3 $\mu \mathrm{m}$, br. 17.2-23.0 $\mu \mathrm{m}$, isth. 6.9-9.2 $\mu \mathrm{m}$, sp. $2.3 \mu \mathrm{m}, \mathrm{lg}$. br. 1.00-1.16 $\times$.

Occur.: 1rrr, 2rrr, 3rrr, 4c, 5rrr, 6c, 8rrr, 9rrr.

Genus: Staurastrum Meyen ex Ralfs emend. Palamar-Mordvintseva 1976

Staurastrum aciculiferum (W. West) Anderss.

var. pulchrum (W. et G. S. West) Först. (Plate 14, Fig. 3-4)

a) Specimens agree with those described in the literature. (Plate 14, Fig. 3)

Lg. 24.1-26.4 $\mu \mathrm{m}$, br. 20.7-23.0 $\mu \mathrm{m}$, br. with pr. 26.4-32.2 $\mu \mathrm{m}$, isth.

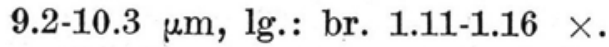

Occur.: 1rrir, 2rrri, 4rri, 5rrr.

b) morpha (Plate 14, Fig. 4)

Cell shape slightly differs from that of encountered typical specimens, as well as from those given by West et al. (1923) and Wysocka (1934). Processes are somewhat divergent, located at the upper angle, apices in most specimens slightly convex. The drawings of similar individuals, but belonging to different taxa, have been found in works of: West et al. (1923; Plate 154, Fig. 11) - ?? St. monticulosum Bréb. ex Ralfs,•Lind (1967) - St. subavicula W. et G. S. West, Förster (1970) - St. aciculiferum var. pulchrum, and Thomasson, Tyler (1971; Fig. 10j) - ? St. aciouliferum.

I think that most of characters of the specimens found are in common with those of St. aciculiferum (= St. monticulosum) var. pulchrum, therefore they were so classified.

Lg. 23.0-28.7 $\mu \mathrm{m}$, br. 20.7-25.3 $\mu \mathrm{m}$, br. with pr. 28.7-37.9 $\mu \mathrm{m}$, isth. 8.0-9.2 $\mu \mathrm{m}$, lg.: br. 1.00-1.13 $\times$.

Occur.: 1rrr, 2rrr, 3rrr, 4c, 5rr, 6cc, 8rrr, 9rrr.

Staurastrum anatinum Cooke et Wills, morpha (Plate 14, Fig. 16)

An appearance of the specimens encountered is most close to the drawing of a small form of St. anatinum from the work of West et al. (1923; Plate 147, Fig. 1). Semicells are more cup-shaped. Their shape agrees more with that of var. pelagicum W. et G. S. West also given in the work of West et al. (1923). In the specimens encountered only one row of inner emarginate verrucae was observed. It is clearly visible in a vertical view of semicells and agrees with drawings of a small form of St. anatinum and not those of var. pelagicum.

Lg. 37.9-39.1 $\mu \mathrm{m}$, br. 25.3-29.9 $\mu \mathrm{m}$, br. with pr. $80.5-82.8 \mu \mathrm{m}$, isth. $12.6 \mu \mathrm{m}$, lg.: br. with pr. $0.47 \times$.

Occur.: 8rirr, 9rir. 
var. brachiatum (Plate 14, Fig. 17-18)

a) Typical specimens, their characters agree with those given in the relevant literature (Plate 14, Fig. 17).

Lg. 16.1-18.4 $\mu \mathrm{m}$, br. with pr. 25.3-32.2 $\mu \mathrm{m}$, isth. 5.7-9.2 $\mu \mathrm{m}$, lg.: br. with pr. 050-0.67 $\times$.

Occur.: 2rrr 6ce, 8rr, 9rri.

b) morpha (Plate 14, Fig. 18)

Cells of an average size, sinus acute-angled, sides nearly straight, apices convex, processes slightly divergent - bifid at the end ultimate divisions bluntly rounded. In a vertical view cells 3-4-angular, sides concave processes narrowing. Thomasson (1963) gives a photograph of a similar specimen.

Morpha cells shape markedly differs from that of typical specimens. West et al. (1923) stress high variation of individual morphology in var. brachiatum. It is possible that the specimens described as morpha constitute some ecological type of var. brachiatum.

Lg. 20.7-27.6 $\mu \mathrm{m}$, br. with pr. 32.2-50.6 $\mu \mathrm{m}$, isth. 10.3-13.8 $\mu \mathrm{m}$, lg.: br. with pr. $0.54-0.64 \times$.

Occur.: 7rrr, 9rrr.

Staurastrum controversum Bréb. ex Bréb.

var. controversum (Plate 15, Fig. 6)

Specimens agree with those given by Hirano (1959b) and Wasylik (1961a), but from those of West et al. (1923) and Rủžička (1973a) differ in smaller number of bifurcate spines.

Lg. 23.0-27.6 $\mu \mathrm{m}$, br. with pr. 34.5-41.4 $\mu \mathrm{m}$, isth. 9.2-11.5 $\mu \mathrm{m}$, lg.: br. with pr. $0.56-0.73 \times$.

\section{Occur.: 4rrr.}

Staurastrum furcatum (Ehrenb. ex Ralfs) Bréb.

var. furcatum f. furcatum (Plate 14, Fig. 5)

Cell shape and dimensions of some individuals agree with those in the description and drawing of St. pseudofurcigerum Reinsch f. minor nob. in the Eichler's work (1896; Plate 4, Fig. 54). In my opinion this taxon should be considered as a synonym of Staurastrum furcatum.

Lg. 20.7-27.6 $\mu \mathrm{m}$, lg. with pr. 25.3-34.5 $\mu \mathrm{m}$, br. 16.1-25.3 $\mu \mathrm{m}$, br. with pr. $24.1-32.2 \mu \mathrm{m}$, isth. $6.9-11.5 \mu \mathrm{m}$, lg. with pr.: br. with pr. 1.00$1.08 \times$.

Occur.: 1rrr, 2rrr, 4r, 5rrr, 6r, 8rrr, 9rrr.

$$
\text { f. spinosum (Bréb.) Nordst. (Plate 14, Fig. 6) }
$$

Lateral processes more massive than apical, other characters close to those in the drawings of Grönblad (1920) and Hirano (1959b). West et al. (1923) include Staurastrum spinosum Bréb. in synonyms of typical variety of Staurastrum furcatum. 
Lg. 25.3-27.6 $\mu \mathrm{m}, \mathrm{lg}$. with pr. $34.5-36.8 \mu \mathrm{m}$, br. $20.7 \mu \mathrm{m}$, br with pr. $34.5-36.8 \mu \mathrm{m}$, isth. $9.2-11.5 \mu \mathrm{m}$, lg. with pr.: br. with pr. $1.00 \times$. Occur.: 7rrr, 9rrr.

Staurastrum hexacerum (Ehrenb. ex Kütz.) Wittr.

var. hexacerum (Plate 15, Fig. 4)

Lg. 23.0-25.3 $\mu \mathrm{m}$, br. with pr. 27.6-29.9 $\mu \mathrm{m}$, isth. 6.9-9.2 $\mu \mathrm{m}$, lg.: br. with pr. $0.81-0.85 \times$.

Occur.: 6rrr, 7rrr, 8rrr, 9rrr.

Staurastrum inflexum Bréb.

var. inflexum (Plate 15, Fig. 5)

In a vertical view semicells quadrangular.

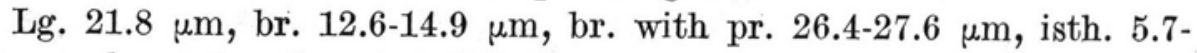
$8.0 \mu \mathrm{m}, \mathrm{lg}$.: br. with pr. $0.79-0.83 \times$.

Occur.: 7rrr, 9rr.

Staurastrum margaritaceum (Ehrenb.) Menegh. ex Ralfs

var. margaritaceum (Plate 14, Fig. 19-20)

Mostly semicells in a vertical view 4-angular, rarely - 3-5-6-angular. Lg. 24.1-32.2 $\mu \mathrm{m}$, br. with pr. 21.8-29.9 $\mu \mathrm{m}$, isth. 6.9-9.2 $\mu \mathrm{m}$, lg.: br with pr. 1.00-1.29 $\times$.

Occur.: 1r, 2rr, 3rrr, 4ce, 5r, 6rr, 7rrr, 8r, 9rr.

Staurastrum oxyacanthum Arch.

var. oxyacanthum (Plate 15, Fig. 7)

Lg. 27.6-33.3 $\mu \mathrm{m}$, br. with pr. 39.1-41.4 $\mu \mathrm{m}$, isth. 10.3-11.5 $\mu \mathrm{m}$, lg.: br. with pr. $0.71-0.81 \times$.

Occur.: 7rrr, 9rrr.

Staurastrum paradoxum Meyen ex Ralfs

var. paradoxum (Plate 14, Fig. 12-15)

The specimens encountered highly vary morphologically. Three groups may be distinguished amongst them:

a) Individuals with cuneate semicells, in a vertical view 4-angular. They fit the drawing of West et al. (1923; Plate 145, Fig. 1) and of Ito (1966b), (Plate 14, Fig. 12)

Plate 14

Fig. 1. Cosmoastrum setigerum var. setigerum, morpha. Fig. 2. Raphidiastrum simonyi var. simonyi. Fig. 3. Staurastrum aciculiferum var. pulchrum. Fig. 4. St. aciculiferum var. pulchrum, morpha. Fig. 5. St. furcatum var. furcatum f. furcatum. Fig. 6. St. furcatum var. furcatum f. spinosum. Fig. 7. Raphidiastrum lunatum var. lunatum. Fig. 8. Raph. granulosum var. granulosum. Fig. 9. Cosmoastrum hystrix var. pannonicum. Fig. 10. Raphidiastrum denticulatum var. denticulatum. Fig. 11. Staurastrum proboscideum var. proboscideum. Fig. 12-15. St. paradoxum var. paradoxum. Fig. 16. St. anatinum, morpha. Fig. 17. St. brachiatum var. brachiatum. Fig. 18. St. brachiatum var. brachiatum, morpha. Fig. 19-20. St. margaritaceum var. margaritaceum. Fig. 21. St. pseudotetracerum var. pseudotetracerum. Fig. 22. St. tetracerum var. tetracerum fac. 2. Fig. 23. St. tetracerum var. tetracerum fac. 3. Fig. 24. St. polymorphum var polymorphum 


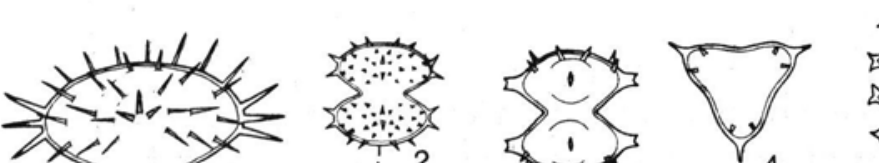

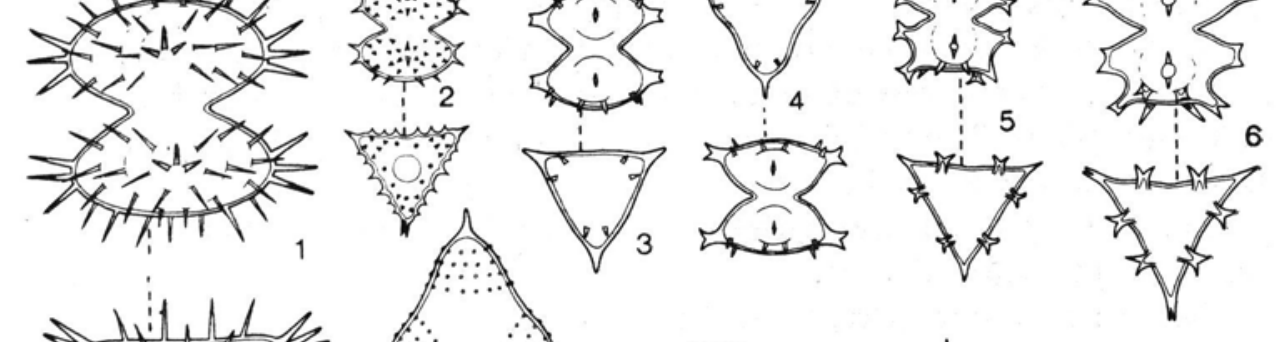

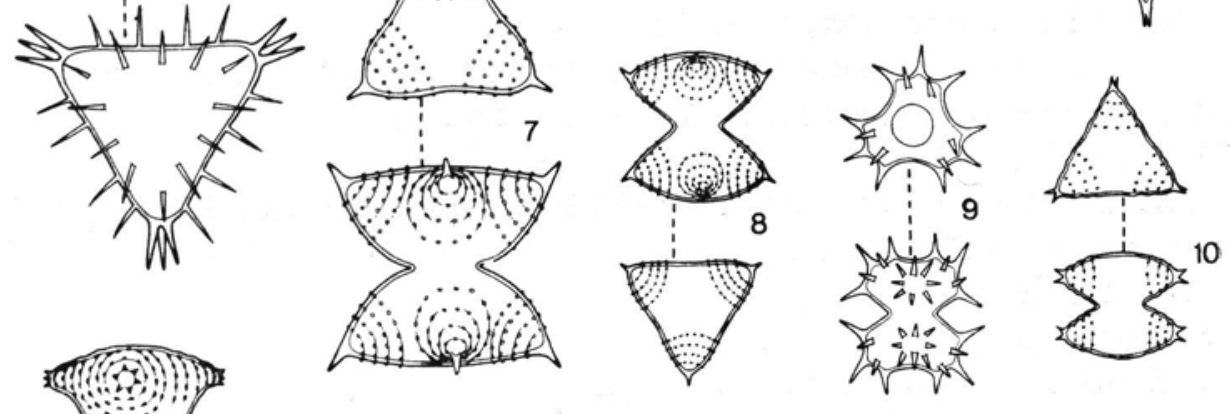
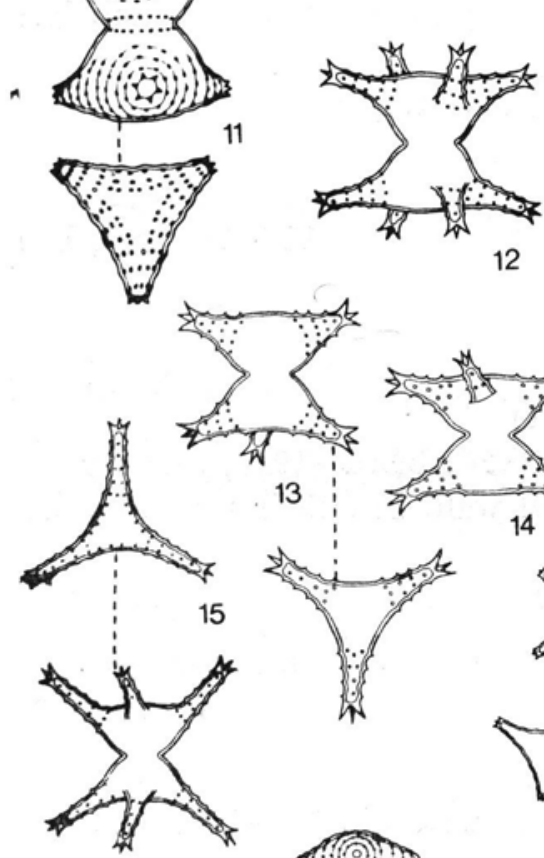

12
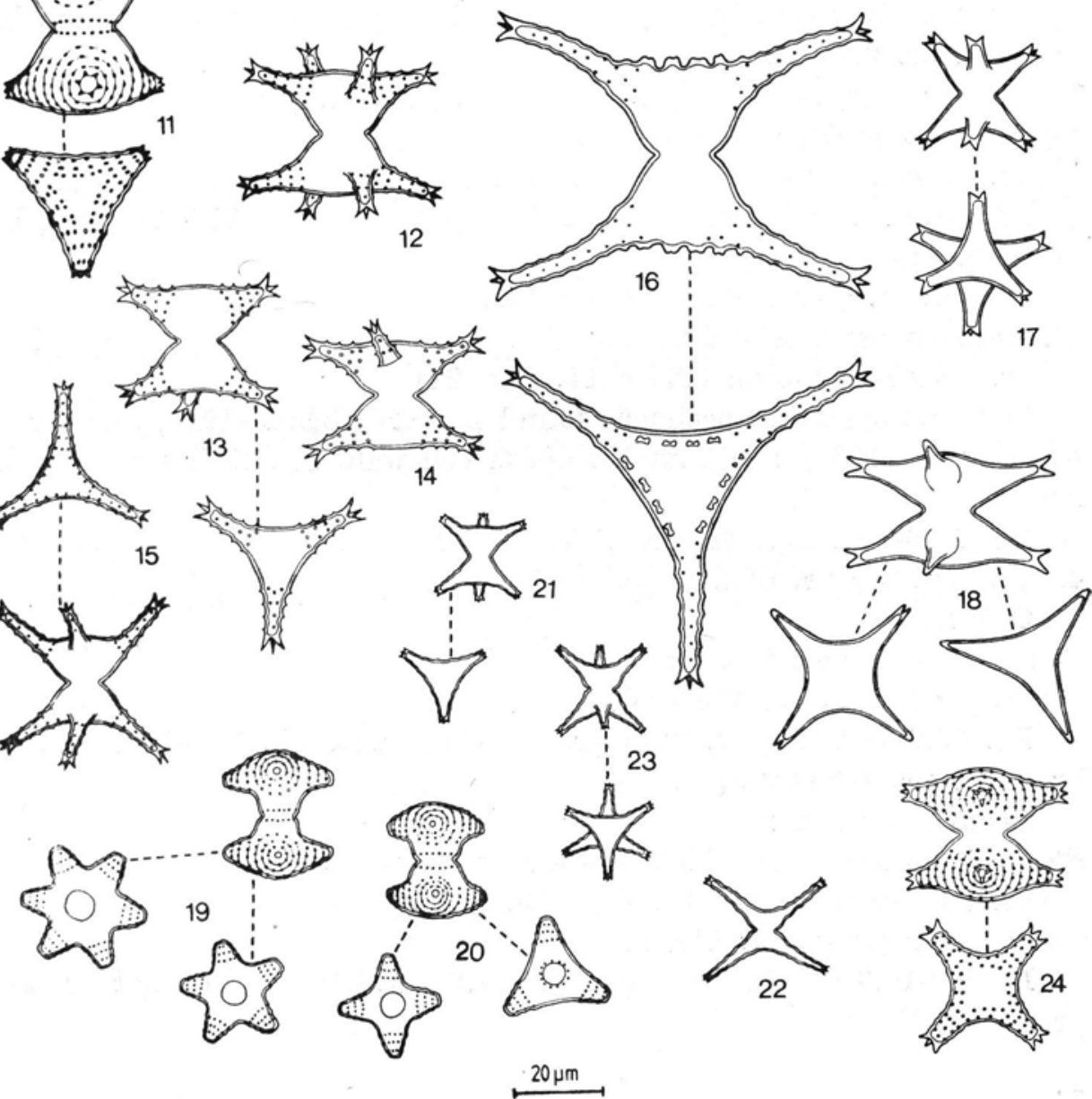
Lg. 27.6-29.9 $\mu \mathrm{m}$, br. with pr. $41.4-44.8 \mu \mathrm{m}$, isth. 9.2-11.5 $\mu \mathrm{m}$, lg.: br. with pr. $0.62-0.72 \times$.

Occur.: 4rrr, 9rrr.

b) Semicells cup-shaped, in a vertical view triangular. Processes of a variable length, a row of short spines on each lateral margin terminated in 3 long spines. They fit a drawing of West et al. (1923; Plate 145, Fig. 4), (Plate 14, Fig. 13-14)

Lg. 23.0-29.9 $\mu \mathrm{m}$, br. with pr. 32.2-49.4 $\mu \mathrm{m}$, isth. 8.0-10.3 $\mu \mathrm{m}$, lg.: br. with pr. $0.58-0.75 \times$.

Occur.: 2rr, 4rrr, 5rrr, 6rrr, 7rrr, 8r, 9rr.

c) Cells small. Processes narrow, markedly divergent. A shape of specimens agrees with a drawing of West et al. (1923; Plate 145, Fig. 2), (Plate 14, Fig. 15)

Lg. 17.2-20.7 $\mu \mathrm{m}$, br. with pr. 35.6-40.2 $\mu \mathrm{m}$, isth. 5.7-8.0 $\mu \mathrm{m}$, lg.: br. with pr. 0.47-0.55 $\times$.

Occur.: 4r.

Staurastrum polymorphum Bréb.

var. polymorphum (Plate 14, Fig. 24)

Lg. 23.0-29.9 $\mu \mathrm{m}$, br. with pr. 24.1-36.8 $\mu \mathrm{m}$, isth. 8.0-11.5 $\mu \mathrm{m}$, lg.: br. with pr. $0.75-1.00 \times$.

Occur.: 1rrr, 2rri, 3rrr, 4ce, 5rr, 6cc, 7rrri, 8rrr, 9rrr.

Staurastrum proboscideum (Bréb.) Arich.

var. proboscideum (Plate 14, Fig. 11)

Lg. 34.5-39.1 $\mu \mathrm{m}$, br. with pr. 33.3-39.1 $\mu \mathrm{m}$, isth. 11.5-13.8 $\mu \mathrm{m}, \mathrm{lg}$.: br. with pr. 1.00-1.06 $\times$.

Occur.: 2rrr, 4rrr, 6rrr, 7r, 8rir, 9rr.

Staurastrum pseudotetracerum (Nordst.) W. et G. S. West

var. pseudotetracerum (Plate 14, Fig. 21)

Cell wall smooth. Specimens found by Grönblad (1948) and Grönblad et al. (1964) also have a smooth cell wall, but their processes are longer.

Lg. 12.6-18.4 $\mu \mathrm{m}$, br. 8.0-11.5 $\mu \mathrm{m}$, br. with pr. 19.5-27.6 $\mu \mathrm{m}$, isth. 4.6-6.9 $\mu \mathrm{m}$, lg.: br. with pr. 0.65-0.72 $\times$.

Occur.: $7 \mathrm{c}$.

Staurastrum sexcostatum Bréb. ex Bréb.

var. productum W. West (Plate 15, Fig. 3)

Lg. 35.6-39.1 $\mu \mathrm{m}$, br. with pr. 40.2-43.7 $\mu \mathrm{m}$, isth. 13.8-16.1 $\mu \mathrm{m}$, lg.: br. with pr. $0.89-0.94 \times$.

Occur.: 8rri, 9rirr.

Staurastrum tetracerum (Kütz.) Ralfs ex Ralfs

var. tetracerum (Plate 14, Fig. 22-23)

a) fac. 2 (Plate 14, Fig. 22)

Lg. 9.2-10.3 $\mu \mathrm{m}$, br. with pr. 26.4-27.6 $\mu \mathrm{m}$, isth. $4.6 \mu \mathrm{m}$, lg.: br. with pr. $0.35-0.37 \times$. 
Occur.: 7rrr, 9rir.

b) fac. 3 (Plate 14, Fig. 23)

Lg. 10.3-11.5 $\mu \mathrm{m}$, br. with pr. 20.7-21.8 $\mu \mathrm{m}$, isth. 5.7-6.9 $\mu \mathrm{m}$, lg.: br. with pr. $0.50-0.56 \times$.

Occur.: 7r, 8rrr, 9rir.

Staurastrum vestitum Ralfs

var. vestitum (Plate 15, Fig. 1)

Semicells cup-shaped. They fit the description of West et al. (1923; Plate 151, Fig. 9-10)

Lg. 32.3-39.1 $\mu \mathrm{m}$, br. 27.6-36.8 $\mu \mathrm{m}$, br. with pr. 59.8-82.8 $\mu \mathrm{m}$, isth. 11.5-16.1 $\mu \mathrm{m}$, lg.: br. with pr. 0.45-0.62 $\times$.

Occur.: 2rr, 3rrr, 4r, 5rrr, 6c, 7rrr, 8rrr, 9rrr.

var. splendidum Grönbl. (Plate 15, Fig. 2)

Lg. 34.5-37.9 $\mu \mathrm{m}$, br. with pr. 64.4-69.0 $\mu \mathrm{m}$, isth. 13.8-17.2 $\mu \mathrm{m}$, lg.: br. with pr. 0.53-0.55 $\times$.

Occur.: 5rrr, 6rrr, 7rrr, 8rrr, 9rrr.

Genus: Bambusina Kützing 1845 nom. cons.

Bambusina brebissonii Kütz. ex Kütz.

var. brebissonii (Plate 15, Fig. 8-10)

a) Cells rather slender. Cell wall sometimes striated (Plate 15, Fig. 10). They fit a description of the typical taxon, (Plate 15, Fig. 9-10)

Lg. 21.8-28.7 $\mu \mathrm{m}$, br. max. 16.1-20.7 $\mu \mathrm{m}$, isth. 13.8-19.5 $\mu \mathrm{m}$, ap. 11.5$13.8 \mu \mathrm{m}$, th. $18.4 \mu \mathrm{m}, \mathrm{lg}$.: br. 1.22-1.43 $\times$.

Occur.: 1ce, 2r, 3r, 4ce, 5rr, 6cc, 7rir, 8r, 9rrr.

b) Cells stout, relatively shorter than those described above. (Plate 15, Fig. 8)

Lg. 21.8-27.6 $\mu \mathrm{m}$, br. max. 21.8-23.0 $\mu \mathrm{m}$, isth. 18.4-21.8 $\mu \mathrm{m}$, ap. 13.816.1 $\mu \mathrm{m}$, lg.: br. 1.00-1.11 $\times$.

Occur.: 4rrr, 6rrr.

var. gracilescens (Nordst.) Wolle (Plate 15, Fig. 11-12)

Lg. 20.7-23.0 $\mu \mathrm{m}$, br. $\max .12 .6-14.9 \mu \mathrm{m}$, isth 11.5-13.8 $\mu \mathrm{m}$, ap. 8.011.5 $\mu \mathrm{m}$, lg.: br. 1.46-1.64 $\times$; zygospore lg. $34.5 \mu \mathrm{m}$, br. $18.4 \mu \mathrm{m}$.

Occur.: 2rirr, 4rrr.

Genus: Desmidium Agardh ex Ralfs 1848

Desmidium cylindricum Grev. ex Nordst.

var. cylindricum (Plate 15, Fig. 13)

Lg. 23.0-27.6 $\mu \mathrm{m}$, br. 39.1-50.6 $\mu \mathrm{m}$, isth. 33.3-46.0 $\mu \mathrm{m}$, ap. 26.4-35.6 $\mu \mathrm{m}$, th. $28.7 \mu \mathrm{m}, \mathrm{lg} .:$ br. $0.48-0.71 \times$.

Occur.: 4rrr, 7rrr, 9rrr. 
Desmidium swartzii (Ag.) Ag. ex Ralfs

var. swartzii (Plate 15, Fig. 14)

Lg. 13.8-19.5 $\mu \mathrm{m}$ br. $35.6-39.1 \mu \mathrm{m}$, isth. 28.7-32.2 $\mu \mathrm{m}$, lg.: br. 0.35$0.53 \times$.

Occur.: 7rrr, 9rrr.

\section{Genus: Hyalotheca Ehrenberg ex Ralfs 1848}

Hyalotheca dissiliens J. E. Smith ex Bréb.

var. dissiliens (Plate 15, Fig. 18-20)

My and Rủžička's (1972) observations indicate high range of cell dimensions.

Cells without papillae in vertical view (fac. circularis - Plate 15, Fig. 18), as well as with three small equidistant papillae (fac. tridentula Plate 15, Fig. 19). Sometimes filaments enclosed in a wide gelatinous sheath (Plate 15, Fig. 20).

Lg. 12.6-23.0 $\mu \mathrm{m}$, br. 18.4-34.5 $\mu \mathrm{m}$, isth. 17.2-31.0 $\mu \mathrm{m}$, lg.: br. $0.62-$ $0.88 \times$.

Occur.: 1rrr, 2rrr, 4r, 5rrr, 6e, 7rrr, 8rirr, 9rrr.

var. minor Delp. (Plate 15, Fig. 22-23)

Cells in vertical view circular - fac. circularis (Plate 15, Fig. 22), or with two opposite papillae fac. bidentula (Plate 15, Fig. 23)

Lg. 16.1-17.2 $\mu \mathrm{m}$, br. 16.1-18.4 $\mu \mathrm{m}$, isth. 14.9-16.1 $\mu \mathrm{m}$, lg.: br. 0.93$1.00 \times$.

Occur.: 1rrr, 2rrr, 3rrr, 4rrr, 6rrr, 7rrr, 8rirr, 9rrr.

var. tatrica Racib. (Plate 15, Fig. 21)

Lg. 18.4-19.5 $\mu \mathrm{m}$, br. 17.2-19.5 $\mu \mathrm{m}$, isth. $16.1 \mu \mathrm{m}$, lg.: br. 1.00-1.07 $\times$.

Occur.: 2r.

? Hyalotheca indica Turn.

var. indica, morpha (Plate 15, Fig. 24)

Cells bigger, excavation at the joints more visible than described by Turner (1892), West et al. (1923), Hirano (1960), Bicudo (1969)

\section{Plate 15}

Fig. 1. Staurastrum vestitum var. vestitum. Fig. 2. St. vestitum var. splendidum. Fig. 3 . St. sexcostatum var. productum. Fig. 4. St. hexacerum var. hexacerum. Fig. 5. St. inflexum var. inflexum. Fig. 6. St. controversum var. controversum. Fig. 7. St. oxyacanthum var. oxyacanthum. Fig. 8-10. Bambusina brebissonii var. brebissonii. Fig. 11-12. B. brebissonii var. gracilescens. Fig. 13. Desmidium cylindricum var. cylindricum. Fig. 14. D. swartzii var. swartzii. Fig. 15. Spondylosium planum var. planum. Fig. 16. S. pulchellum var. pulchellum. Fig. 17. Teilingia excavata var. excavata. Fig. 18-20. Hyalotheca dissiliens var. dissiliens. Fig. 21. H. dissiliens var. tatrica. Fig. 22-23. H. dissiliens var. minor. Fig. 24. ? H. indica var. indica, morpha. Fig. 25. H. mucosa var. mucosa 


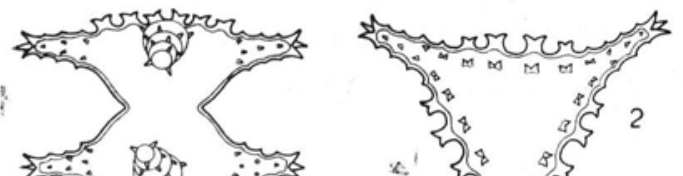

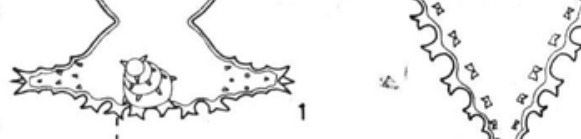
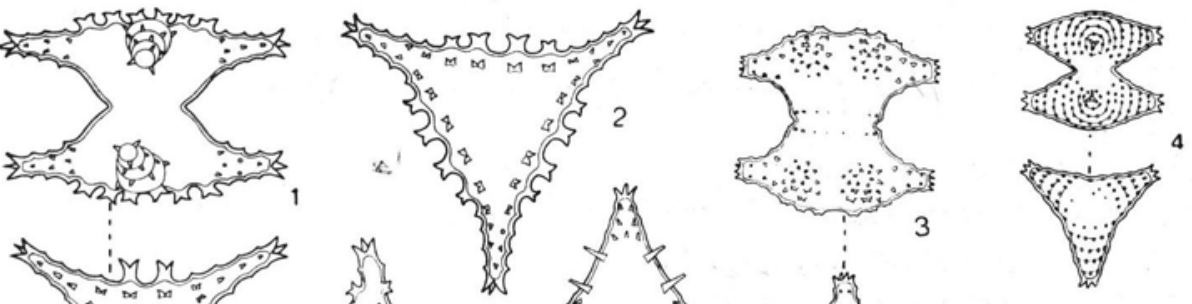

2के
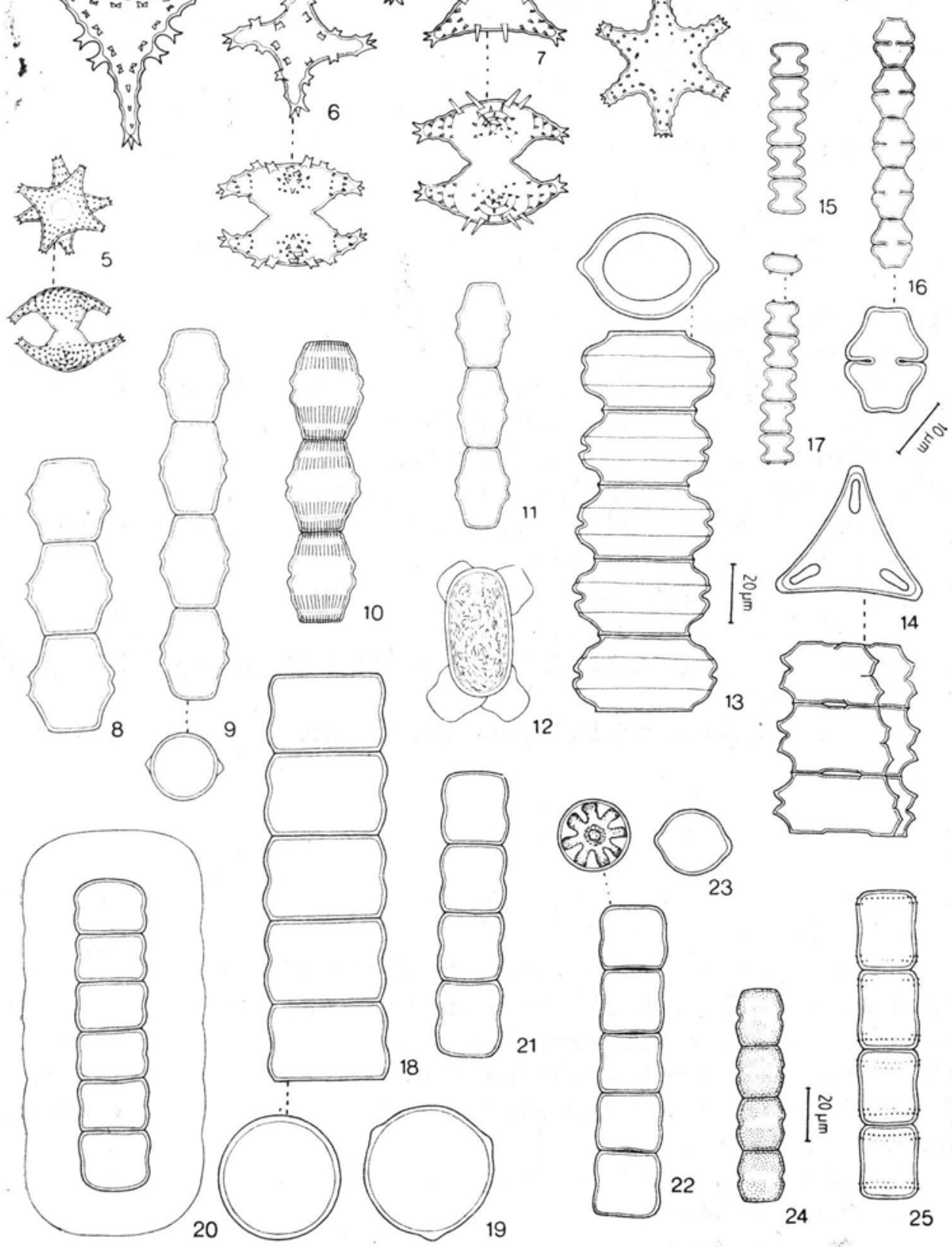
and Förster (1972). They are similar in their shape and dimensions to Hyalotheca dissiliens J. E. Smith ex Bréb. var. hians Wolle, but they differ from them in their cell wall sculpture and body proportions.

Lg. 19.5-21.8 $\mu \mathrm{m}$, br. 18.4-19.5 $\mu \mathrm{m}$, isth. 16.1-18.4 $\mu \mathrm{m}$, lg.: br. 1.06$1.18 \times$.

Occur.: 2rir.

Hyalotheca mucosa (Mert.) Ehrienb. ex Ralfs

var. mucosa (Plate 15, Fig. 25)

Cells cylindrical, slightly constricted in the midregion. They fit Prescott's (1940) drawing.

Lg. 19.5-21.8 $\mu \mathrm{m}$, br. 16.1-17.2 $\mu \mathrm{m}$, isth. 14.9-16.1 $\mu \mathrm{m}$, lg.: br. 1.13$1.35 \times$.

Occur.: 7rrr, 9rrr.

\section{Genus: Spondylosium Brébisson ex Kützing 1849}

Spondylosium planum (Wolle) W. et G. S. West

var. planum (Plate 15, Fig. 15)

Cells smaller, sinus wider open, than it is given by West et al. (1923). Both dimensions and shape agree with those in drawings of: Scott, Grönblad (1957; Plate 35, Fig. 7), Hirano (1960), Bourrelly (1966), Bharati, Pai (1972) and Agarker, Agarkar (1977).

Lg. 8.0-9.2 $\mu \mathrm{m}$, br. 9.2-11.5 $\mu \mathrm{m}$, isth. 4.6-6.9 $\mu \mathrm{m}$, lg.: br. 0.78-1.00 $\times$.

Occur.: 2rrr, 3rrr, 4rrr, 7rr, 8rrr, 9rrr. Spondylosium pulchellum (Arch.) Arch.

var. pulchellum (Plate 15, Fig. 16)

Lg. 11.5-17.2 $\mu \mathrm{m}$, br. 11.5-13.8 $\mu \mathrm{m}$, isth. 2.3-4.6 $\mu \mathrm{m}$, ap. 5.7-9.2 $\mu \mathrm{m}$, lg.: br. $1.00-1.25 \times$.

Occur.: 1rrr, 2rrr, 3rrr, 4r, 5c, 6r, 7rrr, 8c, 9rrr.

\section{Genus: Teilingia Bourelly 1964}

Teilingia excavata (Ralfs ex Ralfs) Bourr.

var. excavata (Plate 15, Fig. 17)

Sometimes, within one filament cells slightly wider than longer were encountered besides cells with the same length and breadth. The former differ in dimentions and length-breadth ratio from those described in the relevant literature (e.g. Migula 1907, 1911, Grönblad et al. 1958, 1968). Whereas Wysocka (1934) describes specimens with the similar dimensions as mine.

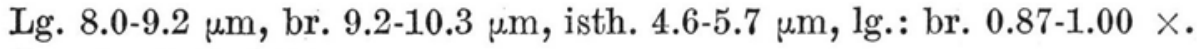
Occur.: 7rrr, 9rrr. 


\section{Acknowledgement}

I would like to thank Prof. Z. Podbielkowski for all the help and guidance. I am grateful to Prof. J. Siemińska and Prof. J. Z. Kadłubowsk a for the critical remarks when the manuscript was prepared for the publication. I would like to express my gratitude to Prof. A. Skirgiełło for the help in the interpretation of some articles of the Int. Cod. Nom. Bot. and to doc. H. Tomaszewicz for the aid in the field.

Grażyna H. Tomaszewicz Department of Phytogeography,

Warsaw University

Al. Ujazdowskie 4, 00-478 Warsaw, Poland 


\section{STRESZCZENIE}

W pracy przedstawiono wykaz gatunków desmidii zebranych w latach 1971-1976 na dziewięciu torfowiskach przejściowych, położonych na obszarze Niziny Srodkowomazowieckiej. Na ośmiu torfowiskach dominowały fitocenozy zespołu Eriophoro-Sphagnetum recurvi, zaś jedno (Grabicz A - stanowisko 7) było typu mszysto-turzycowego z roślinnością nawiązującą do zbiorowisk z klasy Scheuchzerio-Caricetea fuscae, z wyraźnie zaznaczającymi się tendencjami rozwojowymi w kierunku zbiorowisk torfowcowych z klasy Oxycocco-Sphagnetea.

W zebranym materiale zidentyfikowano 226 jednostek taksonomieznych desmidii reprezentujących 20 rodzajów. Poza tym stwierdzono występowanie 16 taksonów z rodziny Mesotaeniaceae należących do 4 rodzajów.

W stosunku do kilkunastu taksonów dokonano pewnych zmian taksonomicznych, mianowicie, przeniesiono do innego rodzaju, zmieniono range w obrębie gatunku, bądź jedno i drugie. Oto wykaz tych taksonów: Mesotaenium endlicherianum Näg. var. endlicherianum f. exiguum (Hansg.) stat. nov., Actinotaenium cucurbita (Bréb. ex Ralfs) Teil. var. cucurbita f. inflatum (Gutw.) comb. nov. et stat. nov., Actinotaenium cucurbitinum (Biss.) Teil. var. minutum (Presc.) Teil. comb. nov. et stat. nov., Actinotaenium wollei (W. et G. S. West) Teil. ex Růž. et Pouz. var wollei f. longior (Grönbl.) comb. nov., Staurodesmus extensus (Anderss.) Teil. var. retusus (Hirano) comb. nov., Staurodesmus ralfsii (W. West) comb. nov., Cosmoastrum hystrix (Ralfs) Pal.-Mordv. var. pannonicum (Lütkem.) comb. nov., Cosmoastrum lapponicum (Schmidle) Pal.-Mordv. comb. nov., Cosmoastrum muricatiforme (Schmidle) comb. nov., Cosmoastrum orbiculare (Ralfs ex Ralfs) Pal.-Mordv. comb. nov., Cosmoastrum teliferum (Ralfs) Pal.-Mordv. var. pecten (Perty) comb. nov., Cosmoastrum turgescens (De Not.) Pal-Mordv. var. sparsigranulatum (Scott et Grönbl.) comb. nov., Cosmoastrum wellipticum (Wille) comb. nov. et nom. nov.

Na podstawie poczynionych obserwacji, jak i danych zawartych w literaturze, krytycznie ustosunkowano się do wydzielenia niektórych gatunków, jak również taksonów wewnątrzgatunkowych. Zaproponowano dokonania pewnych korekt w diagnozach, uznania za synonimy, utrzymania dotychezas przypisywanej rangi. 


\section{LITERATURE}

Agarkar D. S., Agarker M. S., Dikshit R., 1979. Desmids from Bandhavgarh, Madhya Pradesh, India. Hydrobiologia 65, 3: 213-223.

Agarker M. S., Agarkar D. S., 1977. Desmids from Pachmarhi, Madhya Pradesh, India. Hydrobiologia 54, 1: 23-32.

Bharati S. G., Pai K. M., 1972. Some Desmids from Kodaikanal Lake, S. India. Phykos 2, 1-2: 27-36.

Bicudo C. E. M., 1969. Contribution to the knowledge of the Desmids of the State of Sao Paulo, Brazil (including a few from the State of Minas Gerais). Nova Hedw. 17, 1-4: 433-549.

Borge 0., 1913. Beiträge zur Algenflora von Șchweden (2) - Die Algenflora um den Torne-Träsksee in Schwedisch-Lappland. Bot. Notiser 1-3.

Bourrelly P., 1966. Les Algues d'eau douce algues vertes. Paris.

Coesel P. F. M., 1975. Bijdragen tot de kennis der Nederlandse Desmidiaceeënflora 3. N. W.-Overijssel (2). Gorteria 7, 12: 207-213.

Coesel P. F. M., 1979a. Desmids of the broads area of N. W.-Overijssel (the Netherlands) I. Acta Bot. Neerl. 28, 4/5: 257-279.

Coesel P. F. M., 1979b. Desmids of the broads area of N. W.-Overijssel (the Netherlands) II. Acta Bot. Neerl. 28, 6: 385-423.

Coesel P. F. M., Hoogendijk E. M. G., 1975. Bijdragen tot der kennis der Nederlandse Desmidiaceeënflora 2. Desmidiaceeën uit het Mosterdveen. Gorteria 7, 8: 123-128.

Croasdale H., Scott A. M., 1976. New or otherwise Interesting Desmids from Northen Australia. Nova Hedw. 27, 3/4: 501-596.

Deflandre G., 1925. Sur l'existence de formes sigmoides paralleles chez plusieurs Closterium. Rev. Algol. 2, 1: 158-163.

Dubois-Tylski Th., 1966. Peuplement Algal d'une Aulnaie a Sphaignes. Bull. Soc. Bot. Nord 19, 4: 180-187.

Dubois-Tylski Th., 1969. Florule algologique d'un marais d'Ardenne. Rev. Algol. 4: 316-325.

Eichler B., 1890. Spis Desmidyj zebranych w okolicy Międzyrzeca. Pam. Fizjogr. 10: 83-92.

Eichler B., 1892. Materyały do flory wodorostów okolic Międzyrzeca. Pam. Fizjogr. 12: $157-169$.

Eichler B., 1896. Materyały do flory wodorostów okolic Międzyrzeca. Pam. Fizjogr. 14: 119-136.

Förster K., 1970. Beitrag zur Desmidieenflora von Süd-Holstein und der Hansestadt Hamburg. Nova Hedw. 20, $1+2$ : 253-411.

Förster K., 1972. Desmidieen aus dem Südosten der Vereinigten Staaten von Amerika. Nova Hedw. 23, $2+3$ : 515-644. 
Gołowin S., 1964. Glony torfowiska Chlebowo (pow. Oborniki, woj. poznańskie).

Fragm. Flor. Geobot. 10, 1: 121-169.

Goto T., 1975. Desmids in Swamps Hirumo-Numa and Nishi-Numa in Akita Prefecture. Studies of Freshwater Algae 1: 14-25.

Grönblad R., 1920. Finnländische Desmidiaceen aus Keuru. Acta Soce Fauna Fl. Fenn. 47, 4: 4-98.

Grönblad R., 1921. New Desmids from Finland and Northern Russia. Acta Soc. Fauna Fl. Fenn. 49, 7: 5-78.

Grönblad R., 1924. Observations on some Desmids. Acta Soc. Fauna Fl. Fenn. 55, 3: $1-16$.

Grönblad R., 1938. Neue und seltene Desmidiaceen. Bot. Notiser 1: 49-66.

Grönblad R., 1942. Algen, hauptsächlich Desmidiaceen aus dem finnischen, norwegischen und schwedischen Lappland. Acta Soc. Sci. Fenn. Nov. Ser. B. 2, 5 : 5-46.

Grönblad R., 1945. De algis brasiliensibus. Acta Soc. Sci. Fenn. Nov. Ser. B. 2, 6: 3-43. Grönblad R., 1948. Freshwater algae from Täcktom träsk. Bot. Notiser 4; 413-424. Grönblad R., 1960. Contributions to the knowledge of the freshwater algae of Italy.

Soc. Sci. Fenn. Comm. Biol. 22, 4 : 1-85.

Grönblad R., 1963. Desmids from Jämtland, Sweden and adjacent Norway. Soc.

Sei. Fenn. Comm. Biol. 26, 1: 1-43.

Grönblad R., Croasdale H., 1971. Desmids from Namibia (SW Africa). Acta

Bot. Fenn. 93: 1-40.

Grönblad R., Prowse G. A., Scott A. M., 1958. Sudanese Desmids. Acta Bot.

Fenn. 58: 1-82.

Grönblad R., Růžička J., 1959. Zur Systematik der Desmidiaceen. Bot. Notiser 112, 2: 205-226.

Grönblad R., Scott A. M., Croasdale H., 1964. Desmids from Uganda and Lake

Victoria, collected by Dr Edna M. Lind. Acta Bot. Fenn. 66: 1-57.

Grönblad R., Scott A. M., Croasdale H., 1968. Desmids from Sierra Leone, tropical West Africa. Acta Bot. Fenn. 78: 1-41.

Grzelewska E., 1974. Glony torfowiska "Bór na Czerwonem". Fragm. Flor. Geobot. 20, 4: 557-562.

Gutwiński R., 1909. Flora Algarum montium Tatrensium. Bull. Acad. Sci. Cracovie. Hansgirg A., 1892. Prodromus der Algenflora von Böhmen. Archiv Naturwiss.

Landesdurchf. Böhmen 8, 4.

Hinode T., 1971. A study on the Desmids of Kurozo, a Sphagnum-moor in Shikoku.

Hikobia 6, 1-2 : 95-130.

Hinode T., 1977. Desmids collected from Miyajima Island Hiroshima Prefecture.

Hikobia 8, 1-2: 71-90.

Hirano.M., 1955. Flora Desmidiarum Japonicarum. Biol. Lab. Kyoto Univ. 1.

Hirano M., 1956. Flora Desmidiarum Japonicarum. Biol. Lab. Kyoto Univ. 2.

Hirano M., 1957a. Flora Desmidiarum Japonicarum. Biol. Lab. Kyoto -Univ. 4. Hirano M., 1957b. Flora Desmidiarum Japonicarum. Biol. Lab. Kyoto Univ. 5. Hirano M., 1959a. Flora Desmidiarum Japonicarum. Biol. Lab. Kyoto Univ. 7. Hirano M., 1959b. Flora Desmidiarum Japonicarum. Biol. Lab. Kyoto Univ. 9. Hirano M., 1960. Flora Desmidiarum Japonicarum. Biol. Lab. Kyoto Univ. 11. Hirano M., 1968. Desmids of Aretic Alaska. Biol. Lab. Kyoto Univ. 21.

Hirano M., 1969. Freshwater algae from Rangtang Himal, Nepal Himalaya. Biol.

Lab. Kyoto Univ. 22: 1-42.

Hirano M., 1972. Desmids from Cambodia, with special reference to Phytoplankton of Lake Grands Lacs (Tonle Sap). Biol. Lab. Kyoto Univ. 23, 3/4: 123-157.

Hirano M., 1974. Freshwater algae from North Borneo. Biol. Lab. Kyoto Univ. 24, 3: 121-144. 
Hirano M, 1979. Freshwater algae from Yukidori Zawa. Mem. Nat. Inst. Polar Res. 11: 1-25.

Huber-Pestalozzi G., 1928. Beiträge zur Kenntnis der Süsswasseralgen von Korsika. Archiv Hydrobiol. 19: 704-718.

Islam A. K. M. N., 1970. Contributions to the knowledge of Desmids of East Pakistan I. Nova Hedw. 20, 3/4: 903-983.

Ito I., 1965a. Desmids flora of Imori-ike of Mt. Myoko. Bull. Jap. Soc. Phycol. 13, 2: 66-71.

Ito I., 1965b. On the distribution of Desmids in Togakushi District, Nagano Prefecture. Bull. Jap. Soc. Phycol. 13, 3: 84-92.

Ito I., 1965c. The freshwater algae study group: The ecological studies of the freshwater algae and Rhizopoda of the Morinji-numa. Gumma Biol. Educ. Soc. 14: 1-6.

Ito I., 1966a. On the distribution of Desmids in the Ôgemi-ike Swamps Nagano Prefecture (I). Bull. Jap. Soc. Phycol. 14, 2: 41-52.

Ito I., 1966b. Desmids from a pond "Hyotan-no-to" on Atsukeshi swamp in eastern Hokkajdo. Gumma Biol. Educ. Soc. 15.

Ito I., 1968. Desmid flora of Gunma Prefecture. Gumma, pp. 1-26.

Ito I., 1974. Desmids of Kirigamine District 3. Bull. Gumma Biol. Educ. Soc. 23: $1-5$.

Ito I., 1978. Notes on the freshwater algae of Gunma Prefecture (4). Sci. Rep. Shibukawa 7 : 26-71.

Kadłubowska J. Z., 1952. Desmidiaceae torfowiska na Marysinie III (Łódź). Acta Soc. Bot. Pol. 21, 3: 425-434.

Kanetsuna Y., 1960. Studies on the diatom- and desmid-flora of the reclaimed paddy-fields by drainage in city of Toyohashi, Aichi Prefecture. Jap. J. Limnol. 21, $2: 73-86$.

K anetsuna Y., 1967. Studies on the diatom- and desmid-flora of the paddy-fields in the two valleys, Kiso-dani and Ina-dani, Nagano Prefecture. Jap. J. Limnol. 28, $2: 68-91$.

Kondo M., 1975. Desmids of Ozegahara Moor. Studies of freshwater algae. 1: 26-33. Kondracki J., 1977. Regiony fizyczno-geograficzne Polski. Wyd. UW, Warszawa. Kossinskaya C. C., 1952. Flora Plantarum Cryptogamarum URSS. vol. 2. Conjugatae (I) - Mesotaeniales et Gonatozygales. Moskva-Leningrad.

Kossinskaya C. C., 1960. Flora Plantarum Cryptogamarum URSS. vol. 5. Conjugatae (II) - Desmidiales. Moskva-Leningrad.

Kozłowski W., 1895. Przyczynek do flory wodorostów okolic Warszawy. Pam. Fizjogr. 13: 65-73.

Krawiecowa A., Borówko Z., Czubiński Z., Filipiszynowa M., Ołtuszew ski W., Szweykowski J., Tobolewski Z., 1954. Bielawskie Błoto - ginące torfowisko atlantyckie Pomorza. Ochr. Przyr. 22: 67-160.

Krieger W., 1933a. Die Desmidiaceen der Deutschen Limnologischen Sunda-Expedition. Archiv Hydrobiol. Suppl. 11: 129-226.

Krieger W., 1933b. Die Desmidiaceen Europas mit Berücksichtigung der aussereuropäischen Arten. In: Rabenhorst's Kryptogamenflora. 13, 1, 1, Leipzig.

Krieger W., 1935. Die Desmidiaceen Europas mit Berücksichtigung der aussereuropäischen Arten. In: Rabenhorst's Kryptogamenflora. 13, 1, 2, Leipzig.

Krieger W., 1937a. Die Desmidiaceen Europas mit Berücksichtigung der aussereuropäischen Arten. In: Rabenhorst's Kryptogamenflora. 13, 1, 3, Leipzig.

Krieger W., 1937b. Die Desmidiaceen Europas mit Berücksichtigung der aussereuropäischen Arten. In: Rabenhorst's Kryptogamenflora. 13, 1, 4, Leipzig.

Krieger W., 1939. Die Desmidiaceen Europas mit Berücksichtigung der aussereuropäischen Arten. In: Rabenhorst's Kryptogamenflora. 13, 2, 5, Leipzig. 
Krieger W., Gerloff J., 1962. Die Gattung Cosmarium. 1. Verlag J. Cramer, Weinheim.

Krieger W., Gerloff J., 1965. Die Gattung Cosmarium. 2. Verlag J. Cramer, Weinheim.

Krieger W., Gerloff J., 1969. Die Gattung Cosmarium. 3/4. Verlag J. Cramer, Weinheim.

Laporte L. J., 1931. Rechérches sur la biologie et la systématique des Desmidiées. Encycl. Biol. 9: 1-150, Paris.

Lenzenweger R., 1965. Beiträge zur Desmidiaceenflora des Ibmermoores. Jb. Ö̈. Mus. Ver. 110: 446-453.

Lenzenweger R., 1967. Beiträge zur Desmidiaceenflora des Ibmermoores. Jb. oö. Mus. Ver. 112: 173-184.

Lenzenweger R., 1970a. Beiträge zur Desmidiaceenflora des Ibmermoores. Jb. Oö. Mus. Ver. 115: 251-266.

Lenzenweger R., 1970b. Beiträge zur Desmidiaceen-Flora von Oberösterreich. Mitt. Bot. Linz 2: 2-14.

Lenzenweger R., 1974. Beitrag zur Desmidiaceen-Flora von West-Borneo. Mitt. Bot. Linz 6, 2: 103-128.

Lenzenweger R., 1976. Einige Desmidiaceen aus Mooren Nordkareliens nebst Beobachtungen an Doppelzygoten. Linzer Biol. Beitr. 8, 1: 63-78.

Lenzenweger R., 1977. Zieralgen aus Lappland. Mikrokosmos 66: 334-337.

Lind E. M., 1967. Some East African Desmids. Nova Hedw. 13, 3-4: 361-387.

Lind E. M., 1971. Some Desmids from Uganda. Nova Hedw. 22, 1-2: 535-585.

Lind E. M., Brook A. J., 1980. A key to the commoner Desmids of the English Lake District. Freshwater Biol. Assoc. Sci. Publ. 42.

Messikommer E., 1968/72. Der Goldenbergweiher und seine Algenflora. Mitt. Naturforsch. Ges Schaffhausen 29: 1-16.

Messikommer E., 1971a. Schweizer Algen aus dem Kanton Zürich. Nova Hedw. 21, 1-4: 605-639.

Messikommer E., 1971b. Zur Kenntnis der Algenflora in der Transfluenzlandschaft zwischen Hombrechtikon und Bubikon. Schweiz. Zeitsch. Hydrol. 33, 1: 138-170. Migula W., 1907. Kryptogamen-Flora von Deutschland, Deutsch-Österreich und der Schweiz. Flora von Deutschland 2, 1, Leipzig.

Migula W., 1911. Die Desmidiazeen. Franck. Verlag, Stuttgart.

Mix M., 1970. Die Desmidiaceen des Zeller Lochs bei Fulda. Mitt. Staatsinst. Allg. Bot. Hamburg 13: 71-92.

Oleksowicz A. S., 1978. Glony epifitonowe pięciu jezior dystroficznych położonych na terenie Borów Tucholskich. AUNC, Biol. 22, Nauk. Mat.-Przyr. 45: 1-66.

Palamar G. M., 1960. Ad floram Mesotaenialium, Gonatozygalium et Desmidialium in paludibus Polessje occidentalis Ukrainae. I. Bot. Mater. Otd. Spor. Rast. 13: 71-87.

Palamar-Mordvintseva G. M., 1970. Morphological changes in the natural population of Staurastrum furcatum (Ehr.) Bréb. Ukr. Bot. Zhurn. 27, 3: 368-370.

Palamar-Mordvintseva G. M., 1975a. Anomalous forms in Desmidiales, their taxonomic and possible phylogenetic significance. Ukr. Bot. Zhurn. 32, 1: 1-10.

Palamar-Mordvintseva G. M., 1975b. Application of the method of taxonomic analysis by E. S. Smirnov in systematics of the genus Sphaerozosma Corda (Desmidiales). Bot. Zhurn. 60: 687-695.

Palamar-Mordvintseva G. M., 1977. New and rare for the Ukrainian SSR representatives of the Euastrum genus (Desmidiales). Ukr. Bot. Zhurn. 34, 6: 583-587.

Palamar-Mordvintseva G. M., 1982. Opredelitel presnovodnykh vodoroslej SSSR. vol. 11, 2. Conjugatophyceae, Desmidiales. Leningrad. 
Parra 0. O., Gonzalez., 1977. Desmidiaceas de Chile III: Desmidiaceas de la Isla de Chiloe. Gayana Botanica 34: 1-103.

Prescott G. W., 1940. A concluding list of Desmids from Isle Royale. Michigan Pap. Mich. Acad. Sci. Arts Lett. 26: 23-29.

Prescott G. W., 1966. Algae of the Panama Canal and its tributaries - II. Conjugales. Phykos 5, 1-2: 1-49.

Raciborski M., 1892. Desmidya zebrane przez dr E. Ciastonia w podróży na około ziemi. Rozprawy Wydz. mat.-przyr. Akad. Umiej. 22: 1-32.

Raciborski M., 1895. Die Desmidieenflora des Tapakoomasees. Flora Allg. Bot. Zeit. 81, 1: 30-35.

Ralfs J., 1848. The British Desmidieae. London.

Roll J., 1923. Materiaux pour servir ả l'etude des algues de la Russie. Les algues Desmidiaceae, trouvées dans les bassins de Laponie et du gouvernement Olonetzky. Wologda.

Rosenberg M., 1944. On the variability of the desmid Xanthidium subhastiferum West. New Phytologist 43, 1: 15-22.

Rủžička J., 1954. Společenstva Krásivek Mezilesni Slati. Čas. Nár. Musea Odd. Přir. 123, 2: 176-183.

Rủžička J., 1955. Bemerkungen zur Systematik der Desmidiaceen. 1-4. Preslia 27 : 253-271.

Rủžička J., 1956. Die Desmidiaceen der Moravice-Quellen (Grosser Kessel, Gesenke). Přir. Sborn. Ostr. Kraje 17, 1: 38-58.

Rủžička J., 1957a. Die Desmidiaceen der oberen Moldau (Böhmerwald). Preslia $29,2: 132-154$.

Rủžička J., 1957b. Desmidiaceen aus dem Quellgebiete auf dem "Malý Děd" (Gesenke). Acta Mus. Silesiae 6, 2: 108-121.

Rủžička J., 1959. Přehled rodu Tetmemorus Ralfs. Preslia 31: 101-113.

R ủžička J., 1962. Closterium limneticum Lemm. 1899. Preslia 34: 176-189.

Rủžičk a J., 1972. Die Zieralgen der Insel Hiddensee. Arch. Protistenk. 114: 453-485.

Růžička J., 1973a. Die Zieralgen des Naturschutzgebietes "Řežabinec" (Südböhmen). Preslia 45: 193-241.

R ůžička J., 1973b. Ǔber einige ganz alltägliche Probleme der Desmidiologie und der Desmidiologen. Nova Hedw. 42: 259-273.

Rủžička J., 1975a. Erwägungen über die Taxonomie der Gattung Closterium I. Preslia 47, 3: 193-210.

Rủžička J., 1975b. Erwägungen über die Taxonomie der Gattung Closterium II. Preslia 47, 4: 289-304.

Rúžička J., 1976. Erwägungen über die Taxonomie der Gattung Closterium III. Preslia 48, 1: 1-16.

Rủžička J., 1977. Die Desmidiaceen Mitteleuropas. 1, 1. E. Schweiz. Verlag, Stuttgart.

R ủžička J., 1981. Die Desmidiaceen Mitteleuropas. 1, 2. E. Schweiz. Verlag, Stuttgart. Ryppowa H., 1927. Glony jeziorek torfowcowych, tzw. sucharów w okolicach Wigier. Arch. Hydrobiol. Ryb. 2, 1/2: 41-64.

Sarim F. M., Faridi M. A. F., 1976. Closterium in Peshawar Valley. Pakistan J. Bot. 8, 2: 221-239.

Scott A. M., Grönblad R., 1957. New and interesting Desmids from the Southeastern United States. Acta Soc. Sci. Fenn. Nov. Ser. B. 2, 8: 3-62.

Scott A. M., Grönblad R., Croasdale H., 1965. Desmids from the Amazon Basin, Brazil, collected by Dr H. Sioli. Acta Bot. Fenn. 69 : 1-94.

Scott A. M., Prescott G. W., 1961. Indonesian Desmids. Hydrobiologia 17: 1-132. Skuja H., 1928. Vorarbeiten zu einer Algenflora von Lettland, IV. Acta Horti Bot. Univ. Latv. 3s, 2/3: 103-218. 
Suxena M., R., Venkateswarlu V., 1968. Desmids from Kashmir. Phykos 7: 165-185.

Taylor W. M. R., 1934. The freshwater algae of Newfoundland. I. Pap. Mich. Acad. Sci. Arts Lett. 19: 217-278.

Teiling E., 1946. Zur Phytoplanktonflora Schwedens. Bot. Notiser 1: 75-88.

Teiling E., 1954. Actinotaenium genus Desmidiacearum resuscitatum. Bot. Notiser $4: 376-426$.

Teiling E., 1967. The desmid genus Staurodesmus. Arkiv Bot. 6, 11: 467-630.

Thomasson K., 1960. Some planktic Staurastra from New Zealand. Bot. Notiser $113,3: 225-245$.

Thomasson K., 1963. Algological Notes 2. An interesting Staurastrum population. Rev. Algol. 1: 93-99.

Thomasson K., 1972. Some planktic Staurastra from New Zealand 2. Svensk Bot. Tidskr. 66: 257-274.

Thomasson K., 1973. Actinotaenium, Cosmarium and Staurodesmus in the plankton of Rotorua Lakes. Svensk Bot. Tidskr. 67: 127-141.

Thomasson K., Tyler P. A., 1971. Taxonomy of Australian freshwater algae 2. Some planktic Staurastra from Tasmania. Nova Hedw. 21: 287-319.

Tomaszewicz G. H., 1973a. Desmids of transitional peats in Konik Stary near Warsaw. Acta Soc. Bot. Pol. 42, 3: 391-408.

Tomaszewicz G. H., 1973b. The typical variety and developmental stages of Micrasterias truncata (Corda) Bréb. Acta Soc. Bot. Pol. 42, 4: 591-598.

Tomaszewicz G. H., 1974. Desmids of a dune-surrounded lake in Zieleniec near Warsaw. Acta Soc. Bot. Pol. 43, 3: 399-419.

Tomaszewicz G. H., 1977. Micrasterias jenneri Ralfs var. simplex W. West developmental stage of M. jenneri Ralfs var. jenneri. Acta Soc. Bot. Pol. 46, 4: $635 \cdot 640$.

Turner W. B., 1892. Algae aquae duleis Indiae orientalis. The freshwater algae (principally Desmidieae) of East India. K. Svenska Vetensk. - Akad. Handl. $25,5: 1-187$.

Wasylik K., 1961a. Glony torfowisk wysokich Kotliny Nowotarskiej, ze szezególnym uwzględnieniem desmidii. Fragm. Flor. Geobot. 7, 1: 215-288.

Wasylik K., 1961b. Desmidiaceen der Moore in der Umgebung von Korvanen in Sodankylä, Finnisch-Lappland. Soc. Scien. Fenn. Comm. Biol. 23, 10: 1-46.

Watanabe M., Prescott G. W., Yamagishi T., 1979. Freshwater algae of Papua New Guinea (2) Desmids from Woitape, Central District. In: Studies on Cryptogams of Papua New Guinea. S. Kurokawa (ed.). Acad. Sci. Book Inc., Tokyo pp. 49-66.

West W., West G. S., 1904. A monograph of the British Desmidiaceae. 1. Ray Soc. London.

West W., West G. S., 1905. A monograph of the British Desmidiaceae, 2. Ray Soc. London.

West W., West G. S., 1908. A monograph of the British Desmidiaceae. 3. Ray Soc. London.

West W., West G. S., 1912. A monograph of the British Desmidiaceae. 4. Ray Soc. London.

West W., West G. S., Carter N., 1923. A monograph of the British Desmidiaceae. 5. Ray Soc. London.

Wołoszyńska J., 1914. V. Studien über das Phytoplankton das Viktoriasees. Hedwigia 55,3 : 184-192.

Wysocka H., 1934. Materiały do flory desmidyj z okolic Warszawy. Acta Soc. Bot. Pol. 11, 1: 119-137.

Yamagishi T., Hirano M., 1973. Some freshwater algae from Cambodia. Biol. Lab. Kyoto Univ. 24, 2: 61-85. 